\title{
NHC-Catalyzed [3+3] Annulation of Thioamides and Modified Enals for the Enantioselective Synthesis of Functionalized Thiazinones
}

\author{
Arghya Ghosh, Soumen Barik, and Akkattu T. Biju* \\ Department of Organic Chemistry, Indian Institute of Science, Bangalore-560012, India \\ E-mail: atbiju@iisc.ac.in
}

\section{Supporting Information}

1. General Information $\quad$ S2

2. General Procedure for the Optimization of the Reaction Conditions $\quad$ S3

3. General Procedure for the Enantioselective Synthesis of Functionalized Thiazinones S5

4. Kinetic Studies for the Determination of Reaction Order $\quad$ S6

5. X-Ray Data of $3 \mathbf{x} \quad$ S9

6. Synthesis and Characterization of Functionalized Thiazinones $\quad$ S11

7. ${ }^{1} \mathrm{H}$ and ${ }^{13} \mathrm{C}$ NMR Spectra of Functionalized Thiazinones $\quad$ S30

8. HPLC Data of Functionalized Thiazinones $\quad$ S62 


\section{General Information}

Unless otherwise specified, all reactions were carried out under an atmosphere of argon in flame-dried reaction vessels with Teflon screw caps. Mesitylene was purchased from commercial sources and stored under argon over $4 \AA$ molecular sieves. The 2- bromoenals were synthesized from the corresponding $\alpha, \beta$-unsaturated aldehydes following the literature procedure. ${ }^{1}$ All the thioamide derivatives were prepared following the literature procedure. ${ }^{2}$ The triazolium salt $\mathbf{C}$ was synthesized following the literature procedure. ${ }^{3} \mathrm{~K}_{2} \mathrm{CO}_{3}$ was purchased from SD-Fine.

Analytical thin layer chromatography was performed on TLC Silica gel 60 F254. Visualization was accomplished with short wave UV light or $\mathrm{KMnO}_{4}$ staining solutions followed by heating. Flash chromatography was performed on silica gel (230-400 mesh) by standard techniques eluting with Pet. Ether-EtOAc solvent system.

All compounds were fully characterized. ${ }^{1} \mathrm{H}$ and ${ }^{13} \mathrm{C}$ NMR spectra were recorded on Bruker AV 400 and Bruker Ultra shield spectrometer in solvents as indicated. Chemical shifts $(\delta)$ are given in ppm. The residual solvent signals were used as references and the chemical shifts converted to the TMS scale $\left(\mathrm{CDCl}_{3}: \delta \mathrm{H}=7.26 \mathrm{ppm}, \delta \mathrm{C}=77.16 \mathrm{ppm}\right)$. Infrared (FT-IR) spectra were recorded on a Perkin Elmer Spectrum BX spectrophotometer, v-max in cm-1. Optical rotations were measured on JASCO P-2000 polarimeter at room temperature using $50 \mathrm{~mm}$ cell of $1 \mathrm{~mL}$ capacity. HRMS (ESI) data were recorded on a Micromass Q-TOF Micro instrument. HPLC analysis was performed on Agilent Technologies 1260 Infinity II with UV detector.

${ }^{1}$ (a) Allen, C. F. H.; Edens, Jr. C. O. Org. Synth. 1945, 25, 92. (b) Li, W.; Li; Wan, Z.-K.; Wu, J.; Massefski, W. Org. Lett. 2007, 9, 4607.

${ }^{2}$ Yan, Z.; Liu, A.; Huang, M.; Liu, M.; Pei, H.; Huang, L.; Yi, H.; Liu, W.; Hu, A. Eur. J. Med. Chem. 2018, 149, 170 .

${ }^{3}$ Zhao, C.-G.; Li, F.-Y.; Wang, J. Angew. Chem., Int. Ed. 2016, 55, 1820. 


\section{General Procedure for the Optimization of the Reaction Conditions}

To an oven-dried Schlenk reaction vessel equipped with a magnetic stir bar was taken the (Z)-2-bromo-3-phenylacrylaldehyde $\mathbf{2 a}(63.4 \mathrm{mg}, 0.30 \mathrm{mmol}, 1.2$ equiv) and benzothioamide 1a (34.3 mg, $0.25 \mathrm{mmol}, 1.0$ equiv) with triazolium salt $(0.025 \mathrm{mmol}, 10 \mathrm{~mol} \%)$, additive (0.05 mmol, $20 \mathrm{~mol} \%)$ and $4 \AA$ MS (100 mg). The mixture was kept under argon atmosphere. To this mixture was added solvent $(2.0 \mathrm{~mL})$ under a positive pressure of argon and stirring the reaction mixture at $25^{\circ} \mathrm{C}$. To this stirring solution was added base $(0.375 \mathrm{mmol}, 1.5$ equiv $)$ and the resulting mixture was stirred at $25{ }^{\circ} \mathrm{C}$ for $72 \mathrm{~h}$ (the reaction temperature was maintained at $25^{\circ} \mathrm{C}$ using a chiller having $\mathrm{MeOH}$ bath maintained at $25^{\circ} \mathrm{C}$ for $72 \mathrm{~h}$ ). Filtration and evaporation of the solvent to obtain the crude product, whose yield was determined by ${ }^{1} \mathrm{H}$ NMR analysis using $\mathrm{CH}_{2} \mathrm{Br}_{2}$ as the internal standard. The enantiomeric ratio was determined by HPLC analysis on a chiral column.

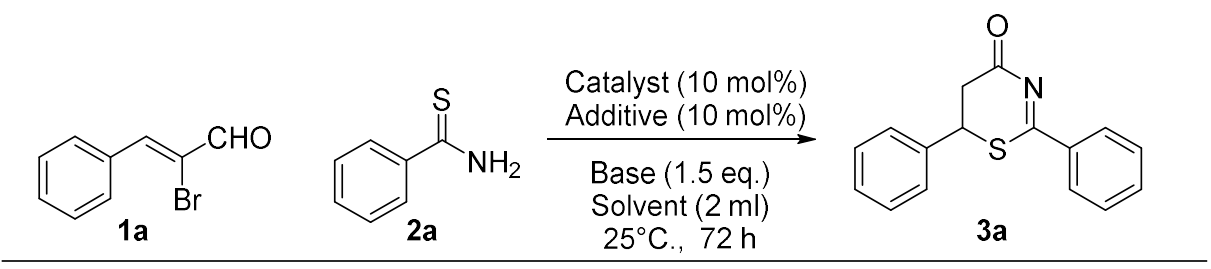

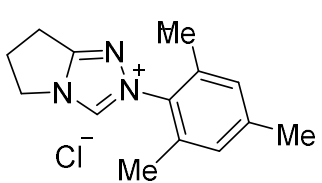

A<smiles>Cc1cc(C)c(-[n+]2cn3c(n2)COC[C@@H]3Cc2ccccc2)c(C)c1</smiles><smiles>Cc1cc(C)c(-[n+]2cn3c(n2)CO[C@@H]2Cc4ccccc4[C@@H]23)c(C)c1</smiles>

B<smiles>Fc1c(F)c(F)c(-[n+]2cn3c(n2)COC[C@@H]3Cc2ccccc2)c(F)c1F</smiles>

E

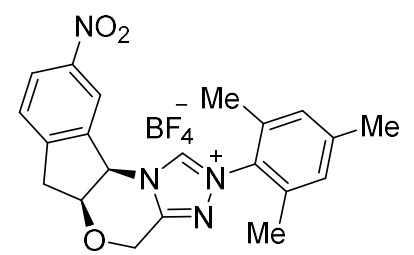

C<smiles>Cc1cc(C)c(-[n+]2cn3c(n2)COC[C@@H]3C(C)C)c(C)c1</smiles>

F 


\begin{tabular}{|c|c|c|c|c|c|c|}
\hline entry & catalyst & base & solvent & additive & yield $(\%)^{b}$ & $\mathrm{er}^{\mathrm{c}}$ \\
\hline 1 & $\mathrm{~A}(10 \mathrm{~mol} \%)$ & $\mathrm{K}_{2} \mathrm{CO}_{3}$ & toluene & LiOAc. $2 \mathrm{H}_{2} \mathrm{O}$ & 28 & nd \\
\hline 2 & $\mathrm{~B}(10 \mathrm{~mol} \%)$ & $\mathrm{K}_{2} \mathrm{CO}_{3}$ & toluene & LiOAc. $2 \mathrm{H}_{2} \mathrm{O}$ & 67 & $87: 13$ \\
\hline 3 & $\mathrm{C}(10 \mathrm{~mol} \%)$ & $\mathrm{K}_{2} \mathrm{CO}_{3}$ & toluene & LiOAc. $2 \mathrm{H}_{2} \mathrm{O}$ & 70 & $92: 8$ \\
\hline 4 & $\mathrm{D}(10 \mathrm{~mol} \%)$ & $\mathrm{K}_{2} \mathrm{CO}_{3}$ & toluene & $\mathrm{LiOAc} .2 \mathrm{H}_{2} \mathrm{O}$ & 72 & $85: 15$ \\
\hline 5 & $\mathrm{E}(10 \mathrm{~mol} \%)$ & $\mathrm{K}_{2} \mathrm{CO}_{3}$ & toluene & $\mathrm{LiOAc} .2 \mathrm{H}_{2} \mathrm{O}$ & $<5$ & nd \\
\hline 6 & $\mathrm{~F}(10 \mathrm{~mol} \%)$ & $\mathrm{K}_{2} \mathrm{CO}_{3}$ & toluene & $\mathrm{LiOAc} .2 \mathrm{H}_{2} \mathrm{O}$ & 87 & $12: 88$ \\
\hline 7 & $\mathrm{C}(10 \mathrm{~mol} \%)$ & $\mathrm{K}_{2} \mathrm{CO}_{3}$ & $o$-xylene & $\mathrm{LiOAc} .2 \mathrm{H}_{2} \mathrm{O}$ & 86 & $88: 12$ \\
\hline 8 & C (10 mol \%) & $\mathrm{K}_{2} \mathrm{CO}_{3}$ & mesitylene & LiOAc. $2 \mathrm{H}_{2} \mathrm{O}$ & 67 & 92:8 \\
\hline 9 & $\mathrm{C}(10 \mathrm{~mol} \%)$ & $\mathrm{K}_{2} \mathrm{CO}_{3}$ & $\begin{array}{l}\text { mesitylene: } \\
\text { hexane }(1: 1)\end{array}$ & LiOAc. $2 \mathrm{H}_{2} \mathrm{O}$ & 42 & $88: 12$ \\
\hline 10 & $\mathrm{C}(10 \mathrm{~mol} \%)$ & $\mathrm{K}_{2} \mathrm{CO}_{3}$ & $\mathrm{Ph}-\mathrm{CF}_{3}$ & LiOAc. $2 \mathrm{H}_{2} \mathrm{O}$ & 87 & $84: 16$ \\
\hline 11 & $\mathrm{C}(10 \mathrm{~mol} \%)$ & $\mathrm{K}_{2} \mathrm{CO}_{3}$ & $\mathrm{Ph}-\mathrm{H}$ & $\mathrm{LiOAc} .2 \mathrm{H}_{2} \mathrm{O}$ & 68 & $85: 15$ \\
\hline 12 & $\mathrm{C}(10 \mathrm{~mol} \%)$ & $\mathrm{K}_{2} \mathrm{CO}_{3}$ & $\mathrm{Ph}-\mathrm{Cl}$ & $\mathrm{LiOAc} .2 \mathrm{H}_{2} \mathrm{O}$ & 88 & $79: 11$ \\
\hline 13 & $\mathrm{C}(10 \mathrm{~mol} \%)$ & $\mathrm{K}_{2} \mathrm{CO}_{3}$ & DME & LiOAc. $2 \mathrm{H}_{2} \mathrm{O}$ & $<5$ & nd \\
\hline 14 & $\mathrm{C}(10 \mathrm{~mol} \%)$ & DBU & mesitylene & LiOAc. $2 \mathrm{H}_{2} \mathrm{O}$ & $<5$ & nd \\
\hline 15 & $\mathrm{C}(10 \mathrm{~mol} \%)$ & $\mathrm{Na}_{2} \mathrm{CO}_{3}$ & mesitylene & $\mathrm{LiOAc} .2 \mathrm{H}_{2} \mathrm{O}$ & 76 & 91:9 \\
\hline 16 & $\mathrm{C}(10 \mathrm{~mol} \%)$ & $\mathrm{Li}_{2} \mathrm{CO}_{3}$ & mesitylene & $\mathrm{LiOAc} .2 \mathrm{H}_{2} \mathrm{O}$ & 10 & $82: 18$ \\
\hline 17 & $\mathrm{C}(10 \mathrm{~mol} \%)$ & $\mathrm{Cs}_{2} \mathrm{CO}_{3}$ & mesitylene & $\mathrm{LiOAc} .2 \mathrm{H}_{2} \mathrm{O}$ & 42 & $84: 16$ \\
\hline 18 & $\mathrm{C}(10 \mathrm{~mol} \%)$ & $\left(\mathrm{NH}_{4}\right)_{2} \mathrm{CO}_{3}$ & mesitylene & LiOAc. $2 \mathrm{H}_{2} \mathrm{O}$ & 36 & $85: 15$ \\
\hline 19 & $\mathrm{C}(10 \mathrm{~mol} \mathrm{\%})$ & $\mathrm{K}_{2} \mathrm{CO}_{3}$ & mesitylene & $\mathrm{NaOAc}$ & 52 & $90: 10$ \\
\hline 20 & $\mathrm{C}(10 \mathrm{~mol} \%)$ & $\mathrm{K}_{2} \mathrm{CO}_{3}$ & mesitylene & KOAc & 45 & $88: 12$ \\
\hline 21 & $\mathrm{C}(10 \mathrm{~mol} \%)$ & $\mathrm{K}_{2} \mathrm{CO}_{3}$ & mesitylene & $\mathrm{LiCl}$ & 45 & $88: 12$ \\
\hline 22 & $\mathrm{C}(10 \mathrm{~mol} \%)$ & $\mathrm{K}_{2} \mathrm{CO}_{3}$ & mesitylene & - & 30 & $85: 15$ \\
\hline 23 & $\mathrm{C}(15 \mathrm{~mol} \%)$ & $\mathrm{K}_{2} \mathrm{CO}_{3}$ & mesitylene & LiOAc. $2 \mathrm{H}_{2} \mathrm{O}$ & 76 & $91: 9$ \\
\hline $24^{\mathrm{d}}$ & $\mathrm{C}(10 \mathrm{~mol} \%)$ & $\mathrm{K}_{2} \mathrm{CO}_{3}$ & mesitylene & LiOAc. $2 \mathrm{H}_{2} \mathrm{O}$ & 85 & $87: 13$ \\
\hline $25^{\mathrm{e}}$ & $\mathrm{C}(10 \mathrm{~mol} \%)$ & $\mathrm{K}_{2} \mathrm{CO}_{3}$ & mesitylene & $\mathrm{LiOAc} .2 \mathrm{H}_{2} \mathrm{O}$ & $<5$ & nd \\
\hline
\end{tabular}

${ }^{a}$ General reaction conditions: $1 \mathbf{a}(0.25 \mathrm{mmol}), \mathbf{2 a}(0.30 \mathrm{mmol})$, cat. (10 mol \%), base (1.5 equiv), solvent $(2.0 \mathrm{~mL})$, $25{ }^{\circ} \mathrm{C}$ and $72 \mathrm{~h}$. ${ }^{\mathrm{b}}$ The yields were determined by ${ }^{1} \mathrm{H}$ NMR analysis of crude product using $\mathrm{CH}_{2} \mathrm{Br}_{2}$ as the internal standard. ${ }^{\mathrm{c}}$ The er value was determined by HPLC analysis on a chiral column. ${ }^{\mathrm{d}}$ The reaction was performed at $30{ }^{\circ} \mathrm{C}$. e The reaction was carried out $15^{\circ} \mathrm{C}$. 


\section{General Procedure for the Enantioselective Synthesis of Functionalized Thiazinones}

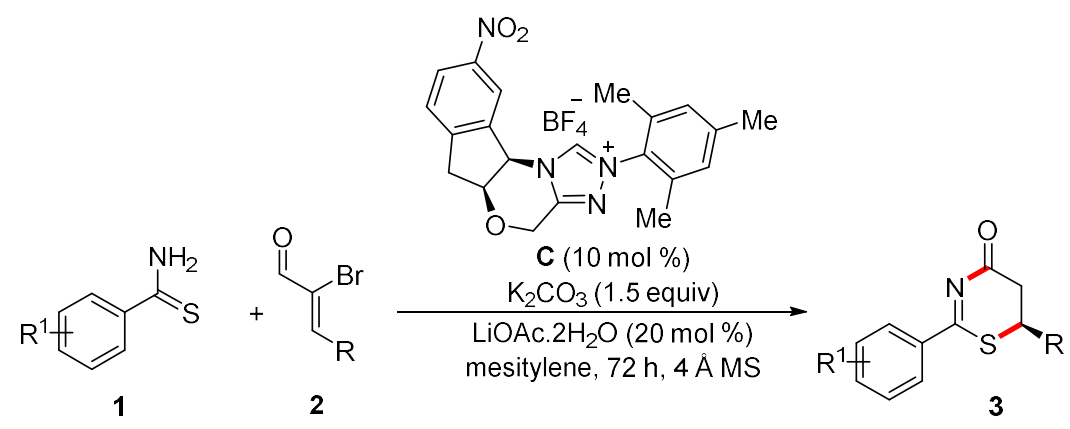

To an oven dried Schlenk reaction vessel equipped with a magnetic stir bar was taken the (Z)-2-bromoenal 2 (63.4 mg, $0.30 \mathrm{mmol}, 1.2$ equiv) and benzothioamide 1 ( $0.25 \mathrm{mmol}, 1.0$ equiv) with triazolium salt $\mathbf{C}(11.6 \mathrm{mg}, 0.025 \mathrm{mmol}, 10 \mathrm{~mol} \%)$, lithium acetate dihydrate $(5.1 \mathrm{mg}, 0.05$ mmol, $20 \mathrm{~mol} \%$ ) and $4 \AA$ MS (100 mg). The mixture was kept under argon atmosphere. To this mixture was added mesitylene $(2.0 \mathrm{~mL})$ under a positive pressure of argon, and the mixture was stirred at $25^{\circ} \mathrm{C}$. To this stirring solution was added $\mathrm{K}_{2} \mathrm{CO}_{3}(52.0 \mathrm{mg}, 0.375 \mathrm{mmol})$ and the resulting mixture was stirred at $25{ }^{\circ} \mathrm{C}$ for $72 \mathrm{~h}$ (the reaction temperature was maintained at $25{ }^{\circ} \mathrm{C}$ using a chiller having $\mathrm{MeOH}$ bath maintained at $25{ }^{\circ} \mathrm{C}$ for $72 \mathrm{~h}$ ). The reaction mixture was purified through silica gel flash column chromatography afforded the thiazinone derivative 3.

All racemic thiazinone derivatives were prepared using either $N$-phenyl triazolium-derived carbenes, $\mathrm{N}$-mesityl triazolium-derived carbenes, or $\mathrm{N}$-mesityl imidazolium-derived carbenes.

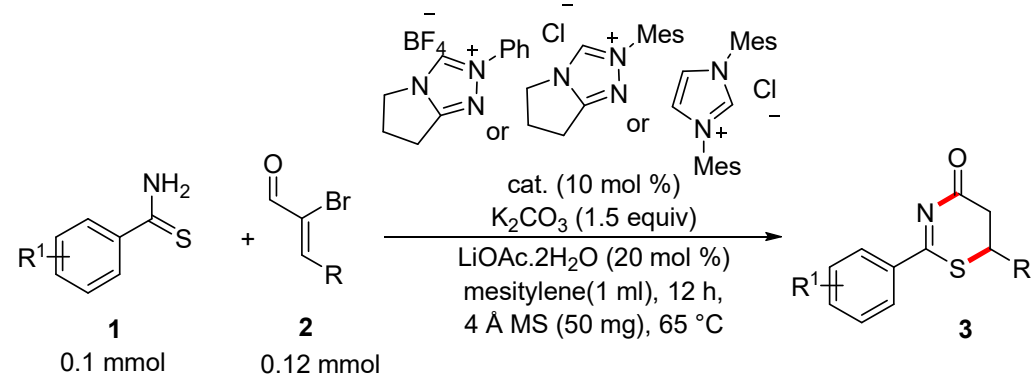

\section{Procedure for the 1 mmol scale experiment}

To an oven dried Schlenk reaction vessel equipped with a magnetic stir bar was taken the (Z)-2-bromo-3-phenylacrylaldehyde $\mathbf{2 a}(253.3 \mathrm{mg}, 1.2 \mathrm{mmol}, 1.2$ equiv) and benzothioamide 1a 
(137.2 mg, $1.0 \mathrm{mmol}, 1.0$ equiv) with triazolium salt C (46.4 mg, $0.10 \mathrm{mmol}, 10 \mathrm{~mol} \%$ ), lithium acetate dihydrate $(20.4 \mathrm{mg}, 0.20 \mathrm{mmol}, 20 \mathrm{~mol} \%)$ and $4 \AA \mathrm{MS}$ (400 mg). The mixture was kept under argon atmosphere. To this mixture was added mesitylene $(8.0 \mathrm{~mL})$ under a positive pressure of argon, and the mixture was stirred at $25^{\circ} \mathrm{C}$. To this stirring solution was added $\mathrm{K}_{2} \mathrm{CO}_{3}(207.3$ $\mathrm{mg}, 1.5 \mathrm{mmol}, 1.5$ equiv) and the resulting mixture was stirred at $25^{\circ} \mathrm{C}$ for $72 \mathrm{~h}$ (the reaction temperature was maintained at $25{ }^{\circ} \mathrm{C}$ using a chiller having $\mathrm{MeOH}$ bath maintained at $25{ }^{\circ} \mathrm{C}$ for $72 \mathrm{~h}$ ). The reaction mixture was purified through silica gel flash column chromatography afforded (S)-2,6-diphenyl-5,6-dihydro-4H-1,3-thiazin-4-one 3a as a pale yellow solid (187 $\mathrm{mg}, 70 \%$ yield, 92:8 er).

\section{Kinetic Studies for the Determination of Reaction $\mathrm{Order}^{4}$}

To an oven-dried Schlenk tube was charged with a magnetic stir-bar, (Z)-2-bromo-3phenylacrylaldehyde 2a and benzothioamide 1a with triazolium salt $\mathbf{C}$, lithium acetate dihydrate, $4 \AA \mathrm{MS}(200 \mathrm{mg})$. Then mesitylene $(4.0 \mathrm{~mL})$ solvent, followed by $\mathrm{K}_{2} \mathrm{CO}_{3}$ was added and stirring the reaction mixture at $25{ }^{\circ} \mathrm{C}$. After a defined time-interval, $100 \mu \mathrm{L}$ of the reaction mixture was taken out from the mixture, filtered and concentrated to obtain crude residue, which was analysed using ${ }^{1} \mathrm{H}$ NMR using equivalent amount of $100 \mu \mathrm{L}$ a standard solution of $\mathrm{CH}_{2} \mathrm{Br}_{2}$ as an external standard.

\footnotetext{
${ }^{4}$ (a) Nguyen, X. B.; Nakano, Y.; Duggan, N. M.; Scott, L.; Breugst, M.; Lupton, D. W. Angew. Chem., Int. Ed. 2019, 58, 11483. (b) Bera, M.; Agasti, S.; Chowdhury, R.; Mondal, R.; Pal, D.; Maiti, D. Angew. Chem. Int. Ed. 2017, 56, 5272. ${ }^{\mathrm{c}}$ The yields were determined by using $0.125 \mathrm{M}$ solution of $\mathrm{CH}_{2} \mathrm{Br}_{2}$ as the standard in $\mathrm{CDCl}_{3}$. ${ }^{\mathrm{d}}$ The yields were determined by using $0.25 \mathrm{M}$ solution of $\mathrm{CH}_{2} \mathrm{Br}_{2}$ as the standard in $\mathrm{CDCl}_{3}$.
} 


\begin{tabular}{|c|c}
\hline \multicolumn{2}{|c}{ Run 1 } \\
\hline time (h) & yield of 3a (\%) \\
\hline 0 & 0 \\
\hline 4 & 8 \\
\hline 6 & 12 \\
\hline 8 & 14 \\
\hline 10 & 17 \\
\hline 12 & 19 \\
\hline 15 & 21 \\
\hline 26 & 36 \\
\hline 39 & 49 \\
\hline 59 & 59 \\
\hline 72 & 70 \\
\hline
\end{tabular}

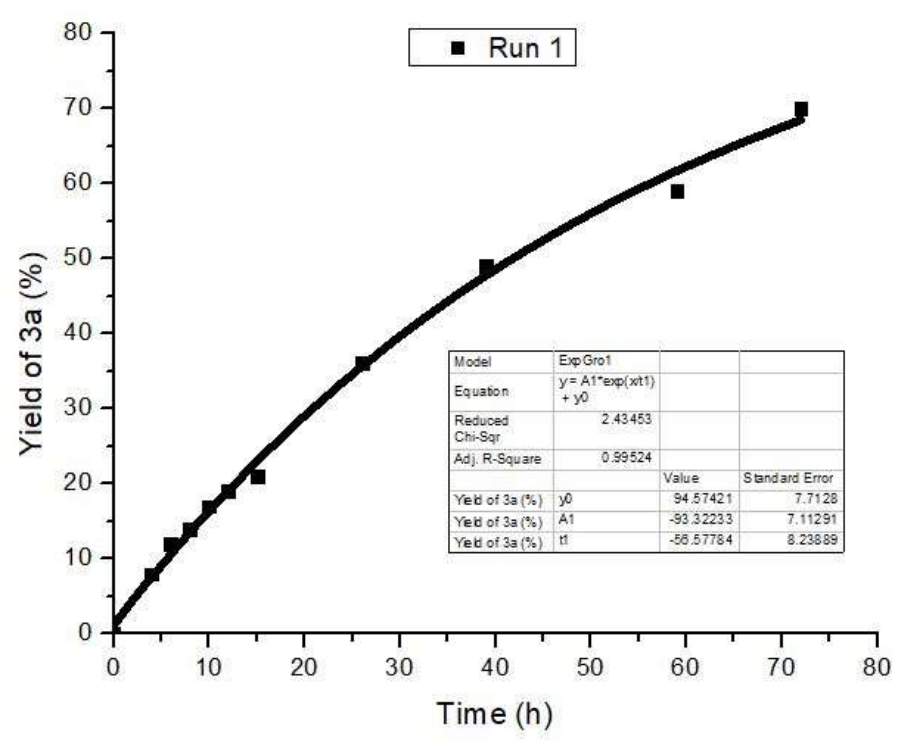

yield of $\mathbf{3 a}$ vs Time

\begin{tabular}{|c|c|}
\hline \multicolumn{2}{|c|}{ Run 2 } \\
\hline time (h) & yield of 3a (\%) $^{\mathrm{d}}$ \\
\hline 0 & 0 \\
\hline 4 & 4 \\
\hline 6 & 6 \\
\hline 8 & 8 \\
\hline 10 & 11 \\
\hline 12 & 13 \\
\hline 15 & 16 \\
\hline 39 & 40 \\
\hline 59 & 48 \\
\hline 72 & 51 \\
\hline
\end{tabular}

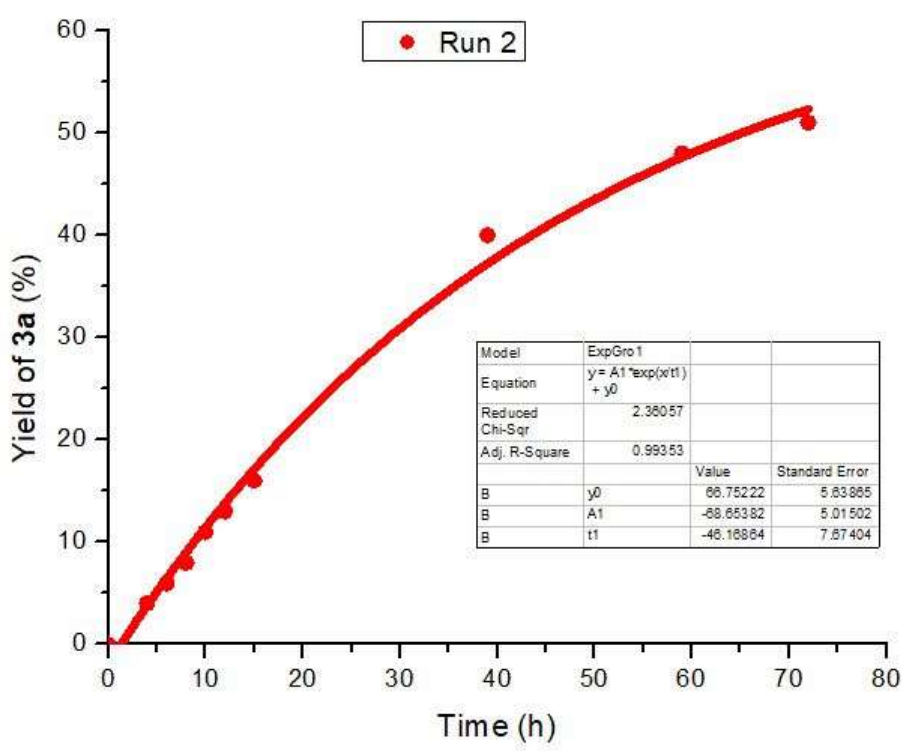

yield of $\mathbf{3 a}$ vs Time

Run 1: 1a (0.50 mmol), 2a (0.60 mmol), C (0.05 mmol), LiOAc. $2 \mathrm{H}_{2} \mathrm{O}(0.10 \mathrm{mmol}), \mathrm{K}_{2} \mathrm{CO}_{3}(0.75$ mmol); Run 2: 1a (1.0 mmol), $2 \mathrm{a}(0.60 \mathrm{mmol}), \mathbf{C}(0.05 \mathrm{mmol}), \mathrm{LiOAc} .2 \mathrm{H}_{2} \mathrm{O}(0.10 \mathrm{mmol}), \mathrm{K}_{2} \mathrm{CO}_{3}$ (0.75 mmol); Run 3: 1a (0.50 mmol), 2a (1.20 mmol), C (0.05 mmol), LiOAc. $2 \mathrm{H}_{2} \mathrm{O}(0.10 \mathrm{mmol})$, $\mathrm{K}_{2} \mathrm{CO}_{3}(1.50 \mathrm{mmol})$. 


\begin{tabular}{|c|c|}
\hline \multicolumn{2}{|c|}{ Run 3 } \\
\hline time (h) & yield of 3a $(\%)^{\mathrm{c}}$ \\
\hline 0 & 0 \\
\hline 4 & 10 \\
\hline 6 & 13 \\
\hline 8 & 16 \\
\hline 10 & 18 \\
\hline 12 & 22 \\
\hline 15 & 24 \\
\hline 25 & 32 \\
\hline 39 & 38 \\
\hline 59 & 49 \\
\hline
\end{tabular}

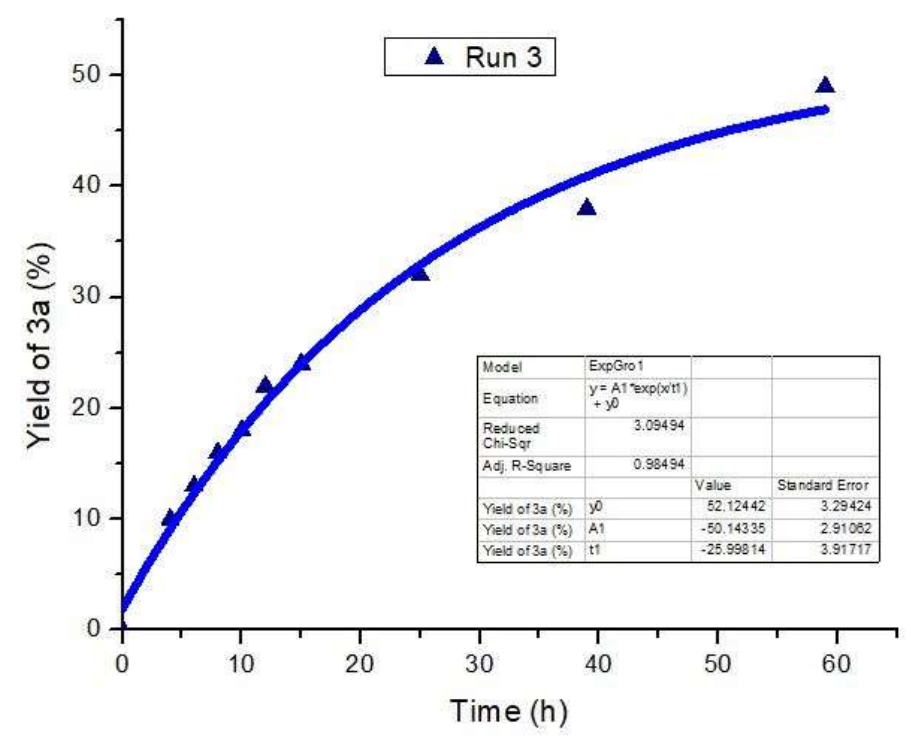

yield of $\mathbf{3 a}$ vs Time

\begin{tabular}{|l|c|c|c|c|c|}
\hline expt. & $\begin{array}{c}\text { thioamide } \\
(\mathbf{1 a})\end{array}$ & bromoenal (2a) & $\begin{array}{c}\text { carbene precursor } \\
(\mathbf{C})\end{array}$ & $\mathrm{LiOAc} .2 \mathrm{H}_{2} \mathrm{O}$ & $\mathrm{K}_{2} \mathrm{CO}_{3}$ \\
\hline Run 1 & $0.50 \mathrm{mmol}$ & $0.60 \mathrm{mmol}$ & $0.05 \mathrm{mmol}$ & $0.10 \mathrm{mmol}$ & $0.75 \mathrm{mmol}$ \\
\hline Run 2 & $1.00 \mathrm{mmol}$ & $0.60 \mathrm{mmol}$ & $0.05 \mathrm{mmol}$ & $0.10 \mathrm{mmol}$ & $0.75 \mathrm{mmol}$ \\
\hline Run 3 & $0.50 \mathrm{mmol}$ & $1.20 \mathrm{mmol}$ & $0.05 \mathrm{mmol}$ & $0.10 \mathrm{mmol}$ & $1.50 \mathrm{mmol}$ \\
\hline
\end{tabular}

\section{Run 1:}

Rate $_{\text {Run } 1}=d y / d x=\left(y_{2}-y_{1}\right) /\left(x_{2}-x_{1}\right)=1.57857$

Rate $_{\text {Run } 1}=1.57857=\mathrm{k}[\text { Thioamide }]^{\mathrm{m}}[\text { Bromoenal }]^{\mathrm{n}}$

\section{Run 2:}

Rate $_{\text {Run2 }}=d y / d x=\left(\mathrm{y}_{2}-\mathrm{y}_{1}\right) /\left(\mathrm{x}_{2}-\mathrm{x}_{1}\right)=1.09286$

Rate $_{\text {Run } 2}=1.09286=\mathrm{k}\left[\right.$ Thioamide $^{\mathrm{m}}\left[{ }_{\text {Bromoenal }}^{\mathrm{n}}\right.$

\section{Run 3:}

Rate $_{\text {Run1 }}=\mathrm{dy} / \mathrm{dx}=\left(\mathrm{y}_{2}-\mathrm{y}_{1}\right) /\left(\mathrm{x}_{2}-\mathrm{x}_{1}\right)=1.75$

Rate $_{\text {Run } 1}=1.75=\mathrm{k}[\text { Thioamide }]^{\mathrm{m}}[\text { Bromoenal }]^{\mathrm{n}}$

Hence from equation (i) and (ii) 
Rate $_{\text {Run2 }} /$ Rate $_{\text {Run } 1}=\mathrm{k}[1.00]^{\mathrm{m}} / \mathrm{k}[0.50]^{\mathrm{m}}$

$(1.09286 / 1.57857)=2^{\mathrm{m}}$

$\log (1.09286 / 1.57857)=\mathrm{m} \log 2$

$\mathrm{m}=-0.53 \sim 0.5$

Order with respect to substrate Thioamide (2a) is (-0.5)

From equation (i) and (iii)

Rate $_{\text {Run3 }} /$ Rate $_{\text {Run } 1}=\mathrm{k}[1.00]^{\mathrm{n}} / \mathrm{k}[0.50]^{\mathrm{n}}$

$(1.75 / 1.57857)=2^{\mathrm{n}}$

$\log (1.75 / 1.57857)=\mathrm{n} \log 2$

$\mathrm{n}=-0.14 \sim 0$

Order with respect to substrate bromoenal (2a) is zero.

\section{X-Ray Data of 3x}

Single crystal of $3 \mathbf{x}$ (recrystallized from $\mathrm{CH}_{2} \mathrm{Cl}_{2} / n$-hexane at $25{ }^{\circ} \mathrm{C}$ ) was mounted and the diffraction data was collected at $296 \mathrm{~K}$ on a Bruker APEX-II CCD diffractometer using SMART/SAINT software. Intensity data were collected using $\operatorname{MoK} \alpha$ radiation $\left(\lambda=0.71073 \mathrm{~A}^{\circ}\right)$. The single crystal was affixed to a Hampton Research cryoloop using Paratone-N oil. Data collection and reduction was performed using Bruker APEX2 and Bruker SAINT, respectively. The structure was solved by direct methods using the SHELX-97 and refined by full-matrix leastsquares on F2. Empirical absorption corrections were applied with SADABS. All Non-hydrogen atoms were refined anisotropically and hydrogen atoms were included in geometric positions. Structure was drawn using Olex-2 and Mercury-3. CCDC 1950784 (3x) contains the supplementary crystallographic data for this paper. These data can be obtained free of charge from Data Centre via www.ccdc.cam.ac.uk/data_request/cif. The crystallographic refinement parameters are given below: 


\begin{tabular}{|c|c|}
\hline Identification code & $3 \mathbf{x}$ \\
\hline Empirical formula & $\mathrm{C}_{16} \mathrm{H}_{12} \mathrm{INOS}$ \\
\hline Formula weight & 393.23 \\
\hline Temperature/K & $296(2)$ \\
\hline Crystal system & Orthorhombic \\
\hline Space group & $\mathrm{P} 22_{1} 2_{1} 2_{1}$ \\
\hline $\mathrm{a} / \AA \AA$ & $9.568(3)$ \\
\hline $\mathrm{b} / \AA \AA$ & $16.041(6)$ \\
\hline $\mathrm{c} / \AA \AA$ & $19.325(7)$ \\
\hline$\alpha /^{\circ} 90$ & 90 \\
\hline$\beta /{ }^{\circ}$ & 90 \\
\hline$\gamma /{ }^{\circ}$ & 90 \\
\hline Volume $/ \AA^{3}$ & $2966.1(18)$ \\
\hline $\mathrm{Z}$ & 8 \\
\hline pcalc $\mathrm{g} / \mathrm{cm}^{3}$ & 1.761 \\
\hline$\mu / \mathrm{mm}^{-1}$ & 2.294 \\
\hline$F(000)$ & 1536.0 \\
\hline Crystal size $/ \mathrm{mm}^{3}$ & $0.25 \times 0.20 \times 0.12$ \\
\hline Radiation & $\operatorname{MoK} \alpha(\lambda=0.71073)$ \\
\hline $2 \Theta$ range for data collection $/{ }^{\circ}$ & 2.9 to 61.1 \\
\hline Index ranges & $-12 \leq \mathrm{h} \leq 13,-22 \leq \mathrm{k} \leq 14,-27 \leq 1 \leq 27$ \\
\hline Reflections collected & 43061 \\
\hline Independent reflections & $5077[$ Rint $=0.0549$, Rsigma $=0.0370]$ \\
\hline Data/restraints/parameters & $8858 / 0 / 361$ \\
\hline Goodness-of-fit on F2 & 1.037 \\
\hline Final $\mathrm{R}$ indexes $[\mathrm{I}>=2 \sigma(\mathrm{I})]$ & $\mathrm{R} 1=0.0370, \mathrm{wR} 2=0.0808$ \\
\hline Final $\mathrm{R}$ indexes [all data] & $\mathrm{R} 1=0.0549, \mathrm{wR} 2=0.0872$ \\
\hline Largest diff. peak/hole / e $\AA^{-3}$ & $1.036 /-0.928$ \\
\hline Flack parameter & $-0.015(8)$ \\
\hline CCDC Number & CCDC 1950784 \\
\hline
\end{tabular}

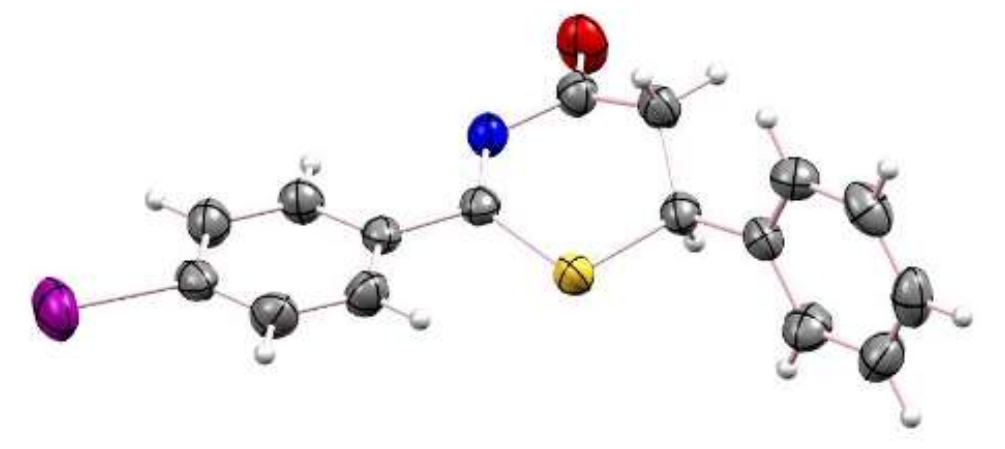

Figure S1. Crystal structure of $\mathbf{3 x}$ (thermal ellipsoids are shown with 50\% probability). 


\section{Synthesis and Characterization of Functionalized Thiazinones}

\section{(S)-2,6-Diphenyl-5,6-dihydro-4H-1,3-thiazin-4-one (3a)}

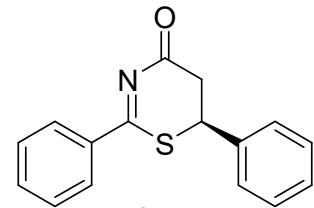

$3 a$

Following the general procedure, treatment of (Z)-2-bromo-3phenylacrylaldehyde 2a (63.4 $\mathrm{mg}, 0.30 \mathrm{mmol})$ and benzothioamide 1a (34.3 $\mathrm{mg}, 0.25 \mathrm{mmol})$ with triazolium salt $\mathbf{C}(11.6 \mathrm{mg}, 0.025 \mathrm{mmol})$, lithium acetate dihydrate $(5.1 \mathrm{mg}, 0.05 \mathrm{mmol}), \mathrm{K}_{2} \mathrm{CO}_{3}(52.0 \mathrm{mg}, 0.375 \mathrm{mmol})$ and $4 \AA \mathrm{MS}$ $(100 \mathrm{mg})$ in mesitylene $(2.0 \mathrm{~mL})$ and stirring the reaction mixture at $25{ }^{\circ} \mathrm{C}$ for $72 \mathrm{~h}$ followed by flash column chromatography (Pet. ether-EtOAc: 85:15) to afford (S)-2,6-diphenyl-5,6-dihydro4H-1,3-thiazin-4-one 3a as a pale yellow solid (45 $\mathrm{mg}, 67 \%$ yield).

$\boldsymbol{R}_{\boldsymbol{f}}($ Pet. ether $/$ EtOAc $=60 / 30): 0.57$; er $=92: 8,[\alpha]_{\mathrm{D}}{ }^{25}=+136.9\left(\mathrm{c} 0.1, \mathrm{CHCl}_{3}\right)$. HPLC $($ Chiralpak IF, 80:20 Hexane / $i$-PrOH, $1.0 \mathrm{~mL} / \mathrm{min}, 254 \mathrm{~nm}$ ) Minor: $18.4 \mathrm{~min}$, Major: $21.7 \mathrm{~min} .{ }^{1} \mathbf{H}$ NMR (400 MHz, $\left.\mathbf{C D C l}_{3}\right) \delta 8.11-8.08(\mathrm{~m}, 2 \mathrm{H}), 7.58-7.56(\mathrm{~m}, 1 \mathrm{H}), 7.47-7.35(\mathrm{~m}, 7 \mathrm{H}), 4.83\left(\mathrm{dd}, J_{1}=12.8\right.$ $\left.\mathrm{Hz}, J_{2}=4.4 \mathrm{~Hz}, 1 \mathrm{H}\right), 3.06\left(\mathrm{dd}, J_{1}=14.2 \mathrm{~Hz}, J_{2}=4.0 \mathrm{~Hz}, 1 \mathrm{H}\right), 3.02\left(\mathrm{dd}, J_{1}=14.1 \mathrm{~Hz}, J_{2}=12.7\right.$ $\mathrm{Hz}, 1 \mathrm{H}) .{ }^{13} \mathbf{C}$ NMR (100 MHz, $\left.\mathbf{C D C l}_{3}\right) \delta 179.28,179.26,137.05,136.43,133.77,129.46,129.11$, 128.84, 127.85, 127.62, 45.10, 37.03. HRMS (ESI) calculated [M+H] for $\mathrm{C}_{16} \mathrm{H}_{14} \mathrm{NOS}: 268.0796$, found: 268.0795. FTIR ( $\left(\mathbf{c m}^{-1}\right)$ 3063, 3028, 2922, 2852, 1713, 1657, 1484, 1378, 1245, 1149, 1077, 905.

(S)-6-(4-Methoxyphenyl)-2-phenyl-5,6-dihydro-4H-1,3-thiazin-4-one (3b)

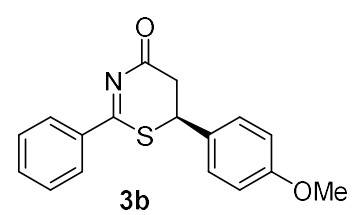

Following the general procedure, treatment of (Z)-2-bromo-3-(4methoxyphenyl)acrylaldehyde $\quad \mathbf{2 b} \quad\left(\begin{array}{llll}72.3 & \mathrm{mg}, & 0.30 & \mathrm{mmol}\end{array}\right)$ and benzothioamide 1a $(34.3 \mathrm{mg}, 0.25 \mathrm{mmol})$ with triazolium salt $\mathbf{C}(11.6 \mathrm{mg}$, $0.025 \mathrm{mmol})$, lithium acetate dihydrate $(5.1 \mathrm{mg}, 0.05 \mathrm{mmol}), \mathrm{K}_{2} \mathrm{CO}_{3}(52.0$ $\mathrm{mg}, 0.375 \mathrm{mmol})$ and $4 \AA \mathrm{MS}(100 \mathrm{mg})$ in mesitylene $(2.0 \mathrm{~mL})$ and stirring the reaction mixture at $25{ }^{\circ} \mathrm{C}$ for $72 \mathrm{~h}$ followed by flash column chromatography (Pet. ether-EtOAc: 80:20) to afford (S)-6-(4-methoxyphenyl)-2-phenyl-5,6-dihydro-4H-1,3-thiazin-4-one $\mathbf{3 b}$ as a pale yellow solid (56 mg, 75\% yield).

$\boldsymbol{R}_{\boldsymbol{f}}($ Pet. ether $/$ EtOAc $=60 / 40): 0.51 ;$ er $=91: 9,[\alpha]_{\mathrm{D}}{ }^{25}=-68.3\left(\mathrm{c} 0.1, \mathrm{CHCl}_{3}\right)$. HPLC $($ Chiralpak IF, 75:25 Hexane / $i$-PrOH, $0.7 \mathrm{~mL} / \mathrm{min}, 254 \mathrm{~nm}$ ) Minor: $27.1 \mathrm{~min}$, Major: $39.2 \mathrm{~min} .{ }^{1} \mathbf{H}$ NMR (400 MHz, $\left.\mathbf{C D C l}_{3}\right) \delta$ 8.09-8.07 (m, 2H), $7.58(\mathrm{t}, J=7.4 \mathrm{~Hz}, 1 \mathrm{H}), 7.47-7.43(\mathrm{~m}, 2 \mathrm{H}), 7.31-7.29$ (m, 2H), 6.94-6.92 (m, 2H), $4.80\left(\mathrm{dd}, J_{1}=12.7 \mathrm{~Hz}, J_{2}=4.1 \mathrm{~Hz}, 1 \mathrm{H}\right), 3.82(\mathrm{~s}, 3 \mathrm{H}), 3.06\left(\mathrm{dd}, J_{1}=\right.$ 
$\left.14.2 \mathrm{~Hz}, J_{2}=4.0 \mathrm{~Hz}, 1 \mathrm{H}\right), 2.95\left(\mathrm{dd}, J_{1}=14.1 \mathrm{~Hz}, J_{2}=12.9 \mathrm{~Hz}, 1 \mathrm{H}\right) .{ }^{13} \mathbf{C}$ NMR (100 MHz, CDCl $\left.\mathbf{3}\right)$ $\delta 179.56,179.54,160.13,136.51,133.74,128.85,127.86,114.82,55.53,44.70,37.34$. HRMS (ESI) calculated $[\mathrm{M}+\mathrm{H}]$ for $\mathrm{C}_{17} \mathrm{H}_{16} \mathrm{NO}_{2} \mathrm{~S}:$ 298.0902, found: 298.0902. FTIR (cm $\left.{ }^{\mathbf{1}}\right)$ 2959, 2923, 2840, 1744, 1713, 1662, 1607, 1511, 1452, 1376, 1249, 1179, 1073.

\section{(S)-2-Phenyl-6-(p-tolyl)-5,6-dihydro-4H-1,3-thiazin-4-one (3c)}

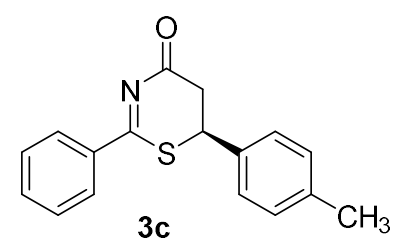

Following the general procedure, treatment of (Z)-2-bromo-3-(ptolyl)acrylaldehyde $2 \mathrm{c}(67.5 \mathrm{mg}, 0.30 \mathrm{mmol})$ and benzothioamide 1a (34.3 mg, $0.25 \mathrm{mmol})$ with triazolium salt $\mathbf{C}(11.6 \mathrm{mg}, 0.025 \mathrm{mmol})$, lithium acetate dihydrate $(5.1 \mathrm{mg}, 0.05 \mathrm{mmol}), \mathrm{K}_{2} \mathrm{CO}_{3}(52.0 \mathrm{mg}, 0.375$ $\mathrm{mmol})$ and $4 \AA \mathrm{MS}(100 \mathrm{mg})$ in mesitylene $(2.0 \mathrm{~mL})$ and stirring the reaction mixture at $25^{\circ} \mathrm{C}$ for $72 \mathrm{~h}$ followed by flash column chromatography (Pet. ether-EtOAc: 85:15) to afford $(S)$-2-phenyl6-(p-tolyl)-5,6-dihydro-4H-1,3-thiazin-4-one $3 \mathbf{c}$ as a yellow oil (46 mg, 64\% yield).

$\boldsymbol{R}_{\boldsymbol{f}}($ Pet. ether $/ \mathrm{EtOAc}=70 / 30): 0.55 ; \mathrm{er}=87: 13,[\alpha]_{\mathrm{D}}{ }^{25}=-88.4\left(\mathrm{c} 0.1, \mathrm{CHCl}_{3}\right)$. HPLC $($ ChiralpakIF, 80:20 Hexane / $i$-PrOH, $0.7 \mathrm{~mL} / \mathrm{min}, 254 \mathrm{~nm})$ Minor: 24.9 min, Major: 31.2 min. ${ }^{1}$ H NMR (400 MHz, $\left.\mathbf{C D C l}_{3}\right) \delta$ 8.10-8.07 (m, 2H), 7.60-7.56 (m, 1H), 7.47-7.43 (m, 2H), 7.28-7.21 (m, 4H), $4.81\left(\mathrm{dd}, J_{1}=12.7 \mathrm{~Hz}, J_{2}=4.1 \mathrm{~Hz}, 1 \mathrm{H}\right), 3.04\left(\mathrm{dd}, J_{1}=14.2 \mathrm{~Hz}, J_{2}=4.2 \mathrm{~Hz}, 1 \mathrm{H}\right), 2.95\left(\mathrm{dd}, J_{1}=\right.$ $\left.14.2 \mathrm{~Hz}, J_{2}=12.6 \mathrm{~Hz}, 1 \mathrm{H}\right), 2.37$ (s, 3H). ${ }^{13} \mathbf{C}$ NMR (100 MHz, $\left.\mathbf{C D C l}_{3}\right) \delta$ 179.48, 139.11, 136.46, 133.97, 130.11, 128.83, 127.84, 127.49, 44.89, 37.13, 21.28..HRMS (ESI) calculated [M+H] for $\mathrm{C}_{17} \mathrm{H}_{16} \mathrm{NOS}: 282.0953$, found: 282.0953.FTIR ( $\left.\mathbf{c m}^{-1}\right)$ 2921, 1713, 1667, 1605, 1510, 1474, 1375 , $1244,1149,904$.

\section{(S)-6-(4-Bromophenyl)-2-phenyl-5,6-dihydro-4H-1,3-thiazin-4-one (3d)}

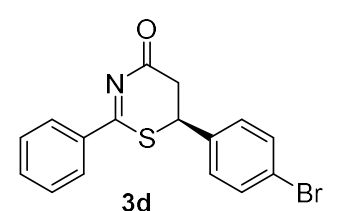

Following the general procedure, treatment of (Z)-2-bromo-3-(4bromophenyl)acrylaldehyde $2 \mathbf{d}(87 \mathrm{mg}, 0.30 \mathrm{mmol})$ and benzothioamide 1a (34.3 mg, $0.25 \mathrm{mmol}$ ) with triazolium salt $\mathbf{C}(11.6 \mathrm{mg}, 0.025 \mathrm{mmol})$, lithium acetate dihydrate $(5.1 \mathrm{mg}, 0.05 \mathrm{mmol}), \mathrm{K}_{2} \mathrm{CO}_{3}(52.0 \mathrm{mg}, 0.375 \mathrm{mmol})$ and 4 $\AA \mathrm{MS}(100 \mathrm{mg})$ in mesitylene $(2.0 \mathrm{~mL})$ and stirring the reaction mixture at $25^{\circ} \mathrm{C}$ for $72 \mathrm{~h}$ followed by flash column chromatography (Pet. ether-EtOAc: 80:20) to afford (S)-6-(4-bromophenyl)-2phenyl-5,6-dihydro-4H-1,3-thiazin-4-one $\mathbf{3 d}$ as a pale yellow solid (72 $\mathrm{mg}, 83 \%$ yield). 
$\boldsymbol{R}_{\boldsymbol{f}}($ Pet. ether $/ \mathrm{EtOAc}=70 / 30): 0.61$; er $=94: 6,[\alpha]_{\mathrm{D}}^{25}=-59.6\left(\mathrm{c} 0.1, \mathrm{CHCl}_{3}\right)$. HPLC $($ Chiralpak IF, 80:20 Hexane / $i$-PrOH, $0.7 \mathrm{~mL} / \mathrm{min}, 254 \mathrm{~nm}$ ) Minor: $26.9 \mathrm{~min}$, Major: $51.1 \mathrm{~min} .{ }^{1} \mathbf{H}$ NMR (400 MHz, $\left.\mathbf{C D C l}_{3}\right) \delta$ 8.09-8.07 (m, 2H), 7.62-7.58 (m, 1H), 7.56-7.52 (m, 2H), 7.48-7.44 (m, 2H), $7.28-7.25(\mathrm{~m}, 2 \mathrm{H}), 4.82\left(\mathrm{dd}, J_{1}=12.3 \mathrm{~Hz}, J_{2}=4.2 \mathrm{~Hz}, 1 \mathrm{H}\right), 3.04\left(\mathrm{dd}, J_{1}=14.0 \mathrm{~Hz}, J_{2}=4.3 \mathrm{~Hz}\right.$, 1H), $2.93\left(\mathrm{dd}, J_{1}=14.0 \mathrm{~Hz}, J_{2}=12.2 \mathrm{~Hz}, 1 \mathrm{H}\right) .{ }^{13} \mathbf{C} \mathbf{N M R}\left(\mathbf{1 0 0} \mathbf{M H z}, \mathbf{C D C l}_{3}\right) \delta 178.78,178.69$, $136.20,136.16,133.86,132.58,129.25,128.84,127.79,123.06,44.38,36.76$. HRMS (ESI) calculated $[\mathrm{M}+\mathrm{H}]$ for $\mathrm{C}_{16} \mathrm{H}_{13} \mathrm{NBrOS}: 345.9901$, found: 345.9904. FTIR (cm $\left.\mathbf{c m}^{-1}\right)$ 2923, 2852, 1713 , 1658, 1569, 1484, 1405, 1373, 1243, 1150, 1072, 1010, 903.

\section{(S)-6-(4-Chlorophenyl)-2-phenyl-5,6-dihydro-4H-1,3-thiazin-4-one (3e)}

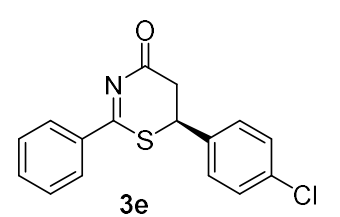

Following the general procedure, treatment of (Z)-2-bromo-3-(4chlorophenyl)acrylaldehyde 2 e $(72.3 \mathrm{mg}, 0.30 \mathrm{mmol})$ and benzothioamide 1a (34.3 mg, $0.25 \mathrm{mmol}$ ) with triazolium salt $\mathbf{C}(11.6 \mathrm{mg}, 0.025 \mathrm{mmol})$, lithium acetate dihydrate $(5.1 \mathrm{mg}, 0.05 \mathrm{mmol}), \mathrm{K}_{2} \mathrm{CO}_{3}(52.0 \mathrm{mg}, 0.375 \mathrm{mmol})$ and 4 $\AA \mathrm{MS}(100 \mathrm{mg})$ in mesitylene $(2.0 \mathrm{~mL})$ and stirring the reaction mixture at $25^{\circ} \mathrm{C}$ for $72 \mathrm{~h}$ followed by flash column chromatography (Pet. ether-EtOAc: 80:20) to afford (S)-6-(4-chlorophenyl)-2phenyl-5,6-dihydro-4H-1,3-thiazin-4-one $3 \mathbf{e}$ as a pale yellow solid (56 mg, 74\% yield).

$\boldsymbol{R}_{\boldsymbol{f}}($ Pet. ether $/ \mathrm{EtOAc}=80 / 20): 0.51$; er $=93: 7,[\alpha]_{\mathrm{D}}{ }^{25}=-59.3\left(\mathrm{c} 0.1, \mathrm{CHCl}_{3}\right)$. HPLC (Chiralpak IF, 80:20 Hexane / $i$-PrOH, $0.7 \mathrm{~mL} / \mathrm{min}, 254 \mathrm{~nm}$ ) Minor: $26.3 \mathrm{~min}$, Major: $45.0 \mathrm{~min} .{ }^{1} \mathbf{H}$ NMR (400 MHz, $\left.\mathbf{C D C l}_{3}\right) \delta$ 8.07-8.05 (m, 2H), 7.60-7.56 (m, 1H), 7.46-7.42 (m, 2H), 7.38-7.35 (m, 2H), 7.31-7.29 (m, 2H), $4.81\left(\mathrm{dd}, J_{1}=12.3 \mathrm{~Hz}, J_{2}=4.1 \mathrm{~Hz}, 1 \mathrm{H}\right), 3.0-3.06(\mathrm{~m}, 1 \mathrm{H}), 2.95-2.88(\mathrm{~m}, 1 \mathrm{H})$. ${ }^{13}$ C NMR (100 MHz, CDCl $) \delta$ 178.83, 178.75, 136.23, 135.64, 134.94, 133.91, 129.61, 128.97, 128.85, 127.80, 44.33, 36.85. HRMS (ESI) calculated [M+H] for $\mathrm{C}_{17} \mathrm{H}_{12} \mathrm{ClNOS}$ : 302.0406, found: 302.0403. FTIR (cm $\left.{ }^{-1}\right)$ 3063, 2960, 2922, 2851, 1714, 1700, 1603, 1490, 1409, 1375, 1243, 1093.

\section{(S)-6-(4-Fluorophenyl)-2-phenyl-5,6-dihydro-4H-1,3-thiazin-4-one (3f)}<smiles>O=C1C[C@H](c2ccc(F)cc2)SC(c2ccccc2)=N1</smiles>

Following the general procedure, treatment (Z)-2-bromo-3-(4fluorophenyl)acrylaldehyde $\quad \mathbf{2 f} \quad(68.7 \quad \mathrm{mg}, \quad 0.30 \quad \mathrm{mmol})$ and benzothioamide 1a $(34.3 \mathrm{mg}, 0.25 \mathrm{mmol})$ with triazolium salt $\mathbf{C}(11.6$ $\mathrm{mg}, 0.025 \mathrm{mmol})$, lithium acetate dihydrate $(5.1 \mathrm{mg}, 0.05 \mathrm{mmol})$, $\mathrm{K}_{2} \mathrm{CO}_{3}(52.0 \mathrm{mg}, 0.375 \mathrm{mmol})$ and $4 \AA \mathrm{MS}(100 \mathrm{mg})$ in mesitylene $(2.0 \mathrm{~mL})$ and stirring the 
reaction mixture at $25^{\circ} \mathrm{C}$ for $72 \mathrm{~h}$ followed by flash column chromatography (Pet. ether-EtOAc: 85:15) to afford (S)-6-(4-fluorophenyl)-2-phenyl-5,6-dihydro-4H-1,3-thiazin-4-one $\mathbf{3 f}$ as a yellow oil (44 mg, 61\% yield).

$\boldsymbol{R}_{\boldsymbol{f}}($ Pet. ether $/$ EtOAc $=70 / 30): 0.56$; er $=94: 6,[\alpha]_{\mathrm{D}}{ }^{25}=-84.3\left(\mathrm{c} 0.1, \mathrm{CHCl}_{3}\right)$. HPLC (Chiralpak IF, 80:20 Hexane / $i$-PrOH, $0.7 \mathrm{~mL} / \mathrm{min}, 254 \mathrm{~nm}$ ) Minor: $23.8 \mathrm{~min}$, Major: $34.1 \mathrm{~min} .{ }^{1} \mathbf{H}$ NMR (400 MHz, $\left.\mathbf{C D C l}_{3}\right) \delta$ 8.08-8.07 (m, 2H), $7.58(\mathrm{t}, 1 \mathrm{H}), 7.45(\mathrm{t}, J=7.3 \mathrm{~Hz}, 2 \mathrm{H})$, 7.37-7.34 (m, 2H), 7.12-7.07 (m, 2H), $4.82\left(\mathrm{dd}, J_{1}=12.4 \mathrm{~Hz}, J_{2}=4.1 \mathrm{~Hz}, 1 \mathrm{H}\right), 3.04\left(\mathrm{dd}, J_{1}=14.1 \mathrm{~Hz}, J_{2}=4.1 \mathrm{~Hz}\right.$, $1 \mathrm{H}), 2.93\left(\mathrm{dd}, J_{1}=14.0 \mathrm{~Hz}, J_{2}=12.4 \mathrm{~Hz}, 1 \mathrm{H}\right) .{ }^{\mathbf{1 3}} \mathbf{C}$ NMR (100 MHz, CDCl $\left.\mathbf{3}\right) \delta 178.91,162.95(\mathrm{~J}$ $=249 \mathrm{~Hz}), 136.37,133.85,133.03,129.43(J=8.3 \mathrm{~Hz}), 128.88,127.86,116.48(J=21.8 \mathrm{~Hz})$, 44.40, 37.19. HRMS (ESI) calculated [M+H] for $\mathrm{C}_{16} \mathrm{H}_{12} \mathrm{FNOS}: 286.0702$, found: 286.0701 . FTIR $\left(\mathbf{c m}^{-1}\right)$ 2960, 2923, 2852, 1714, 1655, 1603, 1509, 1475, 1227,1157, 1099, 905.

\section{(S)-2-Phenyl-6-(4-(trifluoromethyl)phenyl)-5,6-dihydro-4H-1,3-thiazin-4-one (3g)}

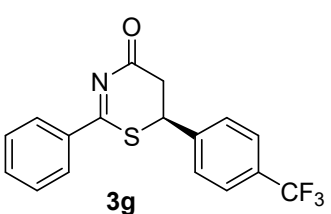

Following the general procedure, treatment of (Z)-2-bromo-3-(4(trifluoromethyl)phenyl)acrylaldehyde $2 \mathrm{~g}(83.7 \mathrm{mg}, 0.30 \mathrm{mmol})$ and benzothioamide 1a (34.3 mg, $0.25 \mathrm{mmol})$ with triazolium salt $\mathbf{C}(11.6 \mathrm{mg}$, $0.025 \mathrm{mmol})$, lithium acetate dihydrate $(5.1 \mathrm{mg}, 0.05 \mathrm{mmol}), \mathrm{K}_{2} \mathrm{CO}_{3}(52.0$ $\mathrm{mg}, 0.375 \mathrm{mmol})$ and $4 \AA \mathrm{MS}(100 \mathrm{mg})$ in mesitylene $(2.0 \mathrm{~mL})$ and stirring the reaction mixture at $25{ }^{\circ} \mathrm{C}$ for $72 \mathrm{~h}$ followed by flash column chromatography (Pet. ether-EtOAc: 80:20) to afford (S)-2-phenyl-6-(4-(trifluoromethyl)phenyl)-5,6-dihydro-4H-1,3-thiazin-4-one $\mathbf{3 g}$ as a pale yellow solid (52 mg, 58\% yield).

$\boldsymbol{R}_{\boldsymbol{f}}($ Pet. ether $/$ EtOAc $=80 / 20): 0.57 ;$ er $=92: 8,[\alpha]_{\mathrm{D}}{ }^{25}=+99.8\left(\mathrm{c} 0.1, \mathrm{CHCl}_{3}\right)$. HPLC $($ Chiralpak IF, 80:20 Hexane / $i$-PrOH, $0.7 \mathrm{~mL} / \mathrm{min}, 254 \mathrm{~nm}$ ) Minor: $17.8 \mathrm{~min}$, Major: $26.4 \mathrm{~min} .{ }^{1} \mathbf{H}$ NMR $\left(400 \mathrm{MHz}, \mathbf{C D C l}_{3}\right) \delta 8.07(\mathrm{~d}, J=7.6 \mathrm{~Hz}, 2 \mathrm{H}), 7.66(\mathrm{~d}, J=8.0 \mathrm{~Hz}, 2 \mathrm{H}), 7.59$ (t, $\left.J=7.4 \mathrm{~Hz}, 1 \mathrm{H}\right)$, $7.52-7.43(\mathrm{~m}, 4 \mathrm{H}), 4.91\left(\mathrm{dd}, J_{1}=11.9 \mathrm{~Hz}, J_{2}=4.4 \mathrm{~Hz}, 1 \mathrm{H}\right), 3.06\left(\mathrm{dd}, J_{1}=14.0 \mathrm{~Hz}, J_{2}=4.3 \mathrm{~Hz}\right.$, 1H), $2.95\left(\mathrm{dd}, J_{1}=14.1 \mathrm{~Hz}, J_{2}=12.1 \mathrm{~Hz}, 1 \mathrm{H}\right) .{ }^{13} \mathbf{C}$ NMR (100 MHz, CDCl $) \delta 178.53,178.40$, 141.26, 136.18, 134.05, 133.90, 131.22 (q, $J=31.7 \mathrm{~Hz}), 128.94,128.90,128.16,128.06,127.85$, 127.62, 126.52, 126.33, 125.14, 122.44, 44.46, 36.63. HRMS (ESI) calculated [M+H] for $\mathrm{C}_{17} \mathrm{H}_{13} \mathrm{~F}_{3} \mathrm{NOS}$ : 336.0670, found: 336.0667. FTIR (cm-1) 2925, 1715, 1679, 1615, 1509, 1474, 1418, 1376, 1326, 1244, 1167, 1119, 1068. 
(S)-6-(4-Nitrophenyl)-2-phenyl-5,6-dihydro-4H-1,3-thiazin-4-one (3h)

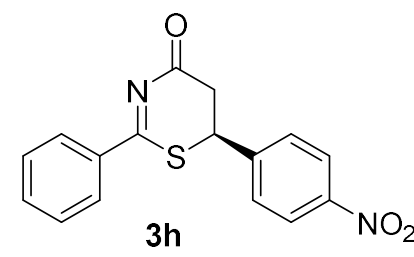

Following the general procedure, treatment of (Z)-2-bromo-3-(4nitrophenyl)acrylaldehyde $\quad \mathbf{2 h} \quad\left(\begin{array}{llll}76.8 & \mathrm{mg}, & 0.30 & \mathrm{mmol})\end{array}\right.$ and benzothioamide 1a (34.3 mg, $0.25 \mathrm{mmol})$ with triazolium salt $\mathbf{C}(11.6$ $\mathrm{mg}, 0.025 \mathrm{mmol})$, lithium acetate dihydrate $(5.1 \mathrm{mg}, 0.05 \mathrm{mmol})$, $\mathrm{K}_{2} \mathrm{CO}_{3}(52.0 \mathrm{mg}, 0.375 \mathrm{mmol})$ and $4 \AA \mathrm{MS}(100 \mathrm{mg})$ in mesitylene $(2.0 \mathrm{~mL})$ and stirring the reaction mixture at $25^{\circ} \mathrm{C}$ for $72 \mathrm{~h}$ followed by flash column chromatography (Pet. ether-EtOAc: 85:15) to afford (S)-6-(4-nitrophenyl)-2-phenyl-5,6-dihydro-4H-1,3-thiazin-4-one $\mathbf{3 h}$ as a brown sticky liquid (41 mg, 52\% yield).

$\boldsymbol{R}_{\boldsymbol{f}}($ Pet. ether $/$ EtOAc $=70 / 30): 0.38$; er $=92: 8,[\alpha]_{\mathrm{D}}{ }^{20}=-88.0\left(\mathrm{c} 0.1, \mathrm{CHCl}_{3}\right)$. HPLC (Chiralpak IA, 65:35 Hexane / $i$-PrOH, $0.7 \mathrm{~mL} / \mathrm{min}, 254 \mathrm{~nm}$ ) Minor: $25.7 \mathrm{~min}$, Major: $35.8 \mathrm{~min} .{ }^{1} \mathbf{H}$ NMR (400 MHz, $\left.\mathbf{C D C l}_{3}\right) \delta$ 8.28-8.25 (m, 2H), 8.09-8.07 (m, 2H), 7.63-7.57 (m, 3H), 7.49-7.46 (m, 2H), $4.96\left(\mathrm{dd}, J_{1}=11.5 \mathrm{~Hz}, J_{2}=4.4 \mathrm{~Hz}, 1 \mathrm{H}\right), 3.09\left(\mathrm{dd}, J_{1}=14.1 \mathrm{~Hz}, J_{2}=4.4 \mathrm{~Hz}, 1 \mathrm{H}\right), 2.97\left(\mathrm{dd}, J_{1}=\right.$ $\left.14.0 \mathrm{~Hz}, J_{2}=11.4 \mathrm{~Hz}, 1 \mathrm{H}\right) .{ }^{13} \mathbf{C}$ NMR (100 MHz, $\left.\mathbf{C D C l}_{\mathbf{3}}\right) \delta 178.04,177.88,148.20,144.45$, 136.05, 134.19, 129.01, 128.77, 127.90, 124.70,44.27, 36.47. HRMS (ESI) calculated [M+H] for $\mathrm{C}_{16} \mathrm{H}_{13} \mathrm{~N}_{2} \mathrm{O}_{3} \mathrm{~S}: 313.0647$, found: 313.0645. FTIR (cm $\left.{ }^{-1}\right)$ 2921, 2852, 1713, 1670, 1602, 1519, 1472, 1392, 1346, 1245, 1152, 904.

\section{(S)-6-(3-Bromophenyl)-2-phenyl-5,6-dihydro-4H-1,3-thiazin-4-one (3i)}

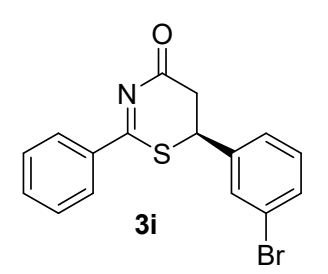

Following the general procedure, treatment of (Z)-2-bromo-3-(3bromophenyl)acrylaldehyde $2 \mathbf{i}(86.9 \mathrm{mg}, 0.30 \mathrm{mmol})$ and benzothioamide

1a (34.3 mg, $0.25 \mathrm{mmol})$ with triazolium salt $\mathbf{C}(11.6 \mathrm{mg}, 0.025 \mathrm{mmol})$, lithium acetate dihydrate $(5.1 \mathrm{mg}, 0.05 \mathrm{mmol}), \mathrm{K}_{2} \mathrm{CO}_{3}(52.0 \mathrm{mg}, 0.375$ $\mathrm{mmol})$ and $4 \AA \mathrm{MS}(100 \mathrm{mg})$ in mesitylene $(2.0 \mathrm{~mL})$ and stirring the reaction mixture at $25^{\circ} \mathrm{C}$ for $72 \mathrm{~h}$ followed by flash column chromatography (Pet. ether-EtOAc: 85:15) to afford (S)-6-(3bromophenyl)-2-phenyl-5,6-dihydro-4H-1,3-thiazin-4-one 3i as a yellow oil (64 $\mathrm{mg}, 74 \%$ yield). $\boldsymbol{R}_{f}($ Pet. ether $/$ EtOAc $=70 / 30): 0.58$; er $=93: 7,[\alpha]_{\mathrm{D}}^{25}=-76.9\left(\right.$ c 0.1, $\left.\mathrm{CHCl}_{3}\right)$. HPLC (Chiralpak IC, 70:30 Hexane / $i$-PrOH, $0.7 \mathrm{~mL} / \mathrm{min}, 254 \mathrm{~nm}$ ) Minor: $47.5 \mathrm{~min}$, Major: 36.5 min. ${ }^{1} \mathbf{H}$ NMR (400 MHz, $\left.\mathbf{C D C l}_{3}\right) \delta$ 8.09-8.07 (m, 2H), 7.61-7.44 (m, 5H), 7.33-7.28 (m, 2H), $4.80\left(\mathrm{dd}, J_{1}=12.4\right.$ $\left.\mathrm{Hz}, J_{2}=4.1 \mathrm{~Hz}, 1 \mathrm{H}\right), 3.04\left(\mathrm{dd}, J_{1}=14.0 \mathrm{~Hz}, J_{2}=4.2 \mathrm{~Hz}, 1 \mathrm{H}\right), 2.92\left(\mathrm{dd}, J_{1}=14.1 \mathrm{~Hz}, J_{2}=12.5\right.$ $\mathrm{Hz}, 1 \mathrm{H}) .{ }^{13} \mathbf{C} \mathbf{N M R}\left(\mathbf{1 0 0} \mathbf{~ M H z}, \mathbf{C D C l}_{3}\right) \delta 178.58,178.56,139.39,136.25,133.92,132.26,130.97$, 
130.77, 130.65, 128.85, 127.83, 126.28, 123.30, 44.43, 36.77. HRMS (ESI) calculated [M+H] for C16H13BrNOS: 345.9901, found: 345.9911. FTIR (cm-1) 2960, 2921, 1713, 1603, 1567, 1475, 1417, 1377, 1244, 1150, 1072, 904.

(S)-6-(3-Chlorophenyl)-2-phenyl-5,6-dihydro-4H-1,3-thiazin-4-one (3j)

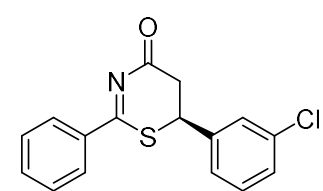

$3 \mathrm{j}$

Following the general procedure, treatment of (Z)-2-bromo-3-(3chlorophenyl)acrylaldehyde $\mathbf{2 j}$ ( $72.3 \mathrm{mg}, 0.30 \mathrm{mmol}$ ) and benzothioamide 1a (34.3 mg, $0.25 \mathrm{mmol})$ with triazolium salt $\mathbf{C}(11.6 \mathrm{mg}, 0.025 \mathrm{mmol})$, lithium acetate dihydrate $(5.1 \mathrm{mg}, 0.05 \mathrm{mmol}), \mathrm{K}_{2} \mathrm{CO}_{3}(52.0 \mathrm{mg}, 0.375 \mathrm{mmol})$ and 4 $\AA$ MS $(100 \mathrm{mg})$ in mesitylene $(2.0 \mathrm{~mL})$ and stirring the reaction mixture at $25^{\circ} \mathrm{C}$ for $72 \mathrm{~h}$ followed by flash column chromatography (Pet. ether-EtOAc: 80:20) to afford $(S)$-6-(3-chlorophenyl)-2phenyl-5,6-dihydro-4H-1,3-thiazin-4-one $\mathbf{3 j}$ as a pale yellow solid (57 $\mathrm{mg}$, 75\% yield).

$\boldsymbol{R}_{\boldsymbol{f}}($ Pet. ether $/$ EtOAc $=70 / 30): 0.62$; er $=93: 7,[\alpha]_{\mathrm{D}}{ }^{20}=-88.0\left(\mathrm{c} 0.1, \mathrm{CHCl}_{3}\right)$. HPLC $($ Chiralpak IC, 70:30 Hexane / $i$-PrOH, $0.7 \mathrm{~mL} / \mathrm{min}, 254 \mathrm{~nm}$ ) Minor: $48.3 \mathrm{~min}$, Major: $35.8 \mathrm{~min} .{ }^{1} \mathbf{H}$ NMR (400 MHz, $\left.\mathbf{C D C l}_{3}\right) \delta$ 8.11-8.09 (m, 2H), 7.64-7.60 (m, 1H), 7.50-7.46 (m, 2H), 7.40-7.34 (m, 3H), 7.31-7.27 (m, 1H), $4.83\left(\mathrm{dd}, J_{1}=12.5 \mathrm{~Hz}, J_{2}=4.2 \mathrm{~Hz}, 1 \mathrm{H}\right), 3.07\left(\mathrm{dd}, J_{1}=14.0 \mathrm{~Hz}, J_{2}=4.1 \mathrm{~Hz}\right.$, 1H), $2.95\left(\mathrm{dd}, J_{1}=14.0 \mathrm{~Hz}, J_{2}=12.5 \mathrm{~Hz}, 1 \mathrm{H}\right) .{ }^{13} \mathbf{C} \mathbf{N M R}\left(\mathbf{1 0 0} \mathbf{~ M H z}, \mathbf{C D C l}_{3}\right) \delta 178.73,178.67$, $139.13,136.24,135.25,133.92,130.75,129.32,128.89,127.85,125.85,44.50,36.78$. HRMS (ESI) calculated [M+H] for $\mathrm{C}_{16} \mathrm{H}_{13}$ CINOS: 302.0406, found: 302.0405. FTIR (cm $\left.{ }^{-1}\right)$ 3063, 2959, 2922, 1713, 1653, 1602, 1570, 1477, 1401, 1243, 1151, 1080, 904.

\section{(S)-6-(3-Nitrophenyl)-2-phenyl-5,6-dihydro-4H-1,3-thiazin-4-one (3k)}

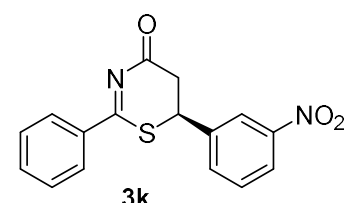

$3 k$

Following the general procedure, treatment of (Z)-2-bromo-3-(3nitrophenyl)acrylaldehyde $\mathbf{2 k}(76.8 \mathrm{mg}, 0.30 \mathrm{mmol})$ and benzothioamide 1a $(34.3 \mathrm{mg}, 0.25 \mathrm{mmol})$ with triazolium salt $\mathbf{C}(11.6 \mathrm{mg}, 0.025 \mathrm{mmol})$, lithium acetate dihydrate $(5.1 \mathrm{mg}, 0.05 \mathrm{mmol}), \mathrm{K}_{2} \mathrm{CO}_{3}(52.0 \mathrm{mg}, 0.375$ $\mathrm{mmol})$ and $4 \AA \mathrm{MS}(100 \mathrm{mg})$ in mesitylene $(2.0 \mathrm{~mL})$ and stirring the reaction mixture at $25^{\circ} \mathrm{C}$ for $72 \mathrm{~h}$ followed by flash column chromatography (Pet. ether-EtOAc: 80:20) to afford $(S)-6-(3-$ nitrophenyl)-2-phenyl-5,6-dihydro-4H-1,3-thiazin-4-one 3k as a pale yellow solid (44 mg, 56\% yield). 
$\boldsymbol{R}_{\boldsymbol{f}}($ Pet. ether $/$ EtOAc $=60 / 40): 0.57$; er $=94: 6,[\alpha]_{\mathrm{D}}{ }^{20}=-122.0\left(\mathrm{c} 0.1, \mathrm{CHCl}_{3}\right)$. HPLC $($ Chiralpak IF, 70:30 Hexane / $i$-PrOH, $0.7 \mathrm{~mL} / \mathrm{min}, 254 \mathrm{~nm}$ ) Minor: $36.3 \mathrm{~min}$, Major: $39.4 \mathrm{~min} .{ }^{1} \mathbf{H}$ NMR (400 MHz, CDCl $\left.\mathbf{l}_{3}\right) \delta 8.28(\mathrm{~s}, 1 \mathrm{H}), 8.24-8.22(\mathrm{~m}, 1 \mathrm{H}), 8.07$ (d, $\left.J=8.0 \mathrm{~Hz}, 2 \mathrm{H}\right), 7.73(\mathrm{~d}, J=7.9$ $\mathrm{Hz}, 1 \mathrm{H}), 7.63-7.58(\mathrm{~m}, 2 \mathrm{H}), 7.47(\mathrm{t}, J=7.6 \mathrm{~Hz}, 2 \mathrm{H}), 4.98\left(\mathrm{dd}, J_{1}=11.7 \mathrm{~Hz}, J_{2}=4.3 \mathrm{~Hz}, 1 \mathrm{H}\right), 3.10$ $\left(\mathrm{dd}, J_{1}=14.0 \mathrm{~Hz}, J_{2}=4.4 \mathrm{~Hz}, 1 \mathrm{H}\right), 3.00\left(\mathrm{dd}, J_{1}=13.9 \mathrm{~Hz}, J_{2}=11.8 \mathrm{~Hz}, 1 \mathrm{H}\right) .{ }^{13} \mathbf{C}$ NMR (100 MHz, $\left.\mathbf{C D C l}_{3}\right) \delta 178.01,177.96,148.83,139.58,136.11,134.09,133.72,130.65,128.98,127.89$, 124.04, 122.81, 44.26, 36.65. HRMS (ESI) calculated [M+H] for $\mathrm{C}_{16} \mathrm{H}_{13} \mathrm{~N}_{2} \mathrm{O}_{3} \mathrm{~S}: 313.0647$, found: 313.0647. FTIR (cm $\left.\mathbf{c m}^{-1}\right)$ 3067, 2923, 1724, 1713, 1655, 1605, 1569, 1528, 1479, 1406, 1350, 1245 , $1153,1097$.

\section{(S)-6-(2-Methoxyphenyl)-2-phenyl-5,6-dihydro-4H-1,3-thiazin-4-one (3I)}<smiles>COc1ccccc1C1CC(=O)N=C(c2ccccc2)S1</smiles>

Following the general procedure, treatment of (Z)-2-bromo-3-(2methoxyphenyl)acrylaldehyde $\quad 21 \quad(72.3 \quad \mathrm{mg}, \quad 0.30 \mathrm{mmole})$ and benzothioamide 1a (34.3 $\mathrm{mg}, 0.25 \mathrm{mmol})$ with triazolium salt $\mathbf{C}(11.6$ $\mathrm{mg}, 0.025 \mathrm{mmol})$, lithium acetate dihydrate $(5.1 \mathrm{mg}, 0.05 \mathrm{mmol}), \mathrm{K}_{2} \mathrm{CO}_{3}$ $(52.0 \mathrm{mg}, 0.375 \mathrm{mmol})$ and $4 \AA \mathrm{MS}(100 \mathrm{mg})$ in mesitylene $(2.0 \mathrm{~mL})$ and stirring the reaction mixture at $25^{\circ} \mathrm{C}$ for $72 \mathrm{~h}$ followed by flash column chromatography (Pet. ether-EtOAc: 85:15) to afford (S)-6-(2-methoxyphenyl)-2-phenyl-5,6-dihydro-4H-1,3-thiazin-4one 31 a pale yellow solid(50 $\mathrm{mg}, 67 \%$ yield).

$\boldsymbol{R}_{\boldsymbol{f}}($ Pet. ether $/$ EtOAc $=70 / 30): 0.41 ;$ er $=85: 15,[\alpha]_{\mathrm{D}}^{25}=+11.0\left(\mathrm{c} 0.1, \mathrm{CHCl}_{3}\right)$. HPLC $($ Chiralpak IF, 70:30 Hexane / $i$-PrOH, $0.7 \mathrm{~mL} / \mathrm{min}, 254 \mathrm{~nm}$ ) Minor: $16.1 \mathrm{~min}$, Major: $18.1 \mathrm{~min} .{ }^{1} \mathbf{H}$ NMR $\left(400 \mathbf{~ M H z}, \mathbf{C D C l}_{3}\right) \delta 8.08(\mathrm{~d}, J=7.8 \mathrm{~Hz}, 2 \mathrm{H}), 7.56(\mathrm{t}, J=7.2 \mathrm{~Hz}, 1 \mathrm{H}), 7.43(\mathrm{t}, J=7.5 \mathrm{~Hz}, 2 \mathrm{H})$, 7.36-7.30(m, 2H), $6.98(\mathrm{t},, J=7.9 \mathrm{~Hz}, 1 \mathrm{H}), 6.92(\mathrm{~d}, J=8.2 \mathrm{~Hz}, 1 \mathrm{H}), 5.31\left(\mathrm{dd}, J_{1}=10.0 \mathrm{~Hz}, J_{2}=\right.$ $5.4 \mathrm{~Hz}, 1 \mathrm{H}), 3.86(\mathrm{~s}, 3 \mathrm{H}), 3.05-2.95(\mathrm{~m}, 2 \mathrm{H}) .{ }^{13} \mathbf{C}$ NMR (100 MHz, CDCl $\left.\mathbf{3}\right) \delta 179.93,179.42$, 156.87, 136.80, 133.48, 129.99, 128.72, 127.81, 127.76, 125.29, 121.16, 111.04, 55.61, 38.54, 35.39..HRMS (ESI) calculated [M+H] for $\mathrm{C}_{17} \mathrm{H}_{16} \mathrm{NO}_{2} \mathrm{~S}: 298.0902$, found: 298.0901 . FTIR (cm 1) $2937,2838,1716,1654,1605,1499,1461,1247,1182,1111,1025,905$. 
(S)-6-(2-Bromophenyl)-2-phenyl-5,6-dihydro-4H-1,3-thiazin-4-one (3m)<smiles>O=C1CC(c2ccccc2Br)SC(c2ccccc2)=N1</smiles>

Following the general procedure, treatment of (Z)-2-bromo-3phenylacrylaldehyde $\mathbf{2 m}(86.9 \mathrm{mg}, 0.30 \mathrm{mmol})$ and benzothioamide 1a (34.3 $\mathrm{mg}, 0.25 \mathrm{mmol})$ with triazolium salt $\mathbf{C}(11.6 \mathrm{mg}, 0.025 \mathrm{mmol})$, lithium acetate dihydrate $(5.1 \mathrm{mg}, 0.05 \mathrm{mmol}), \mathrm{K}_{2} \mathrm{CO}_{3}(52.0 \mathrm{mg}, 0.375$ mmol) and $4 \AA$ MS $(100 \mathrm{mg})$ in mesitylene $(2.0 \mathrm{~mL})$ and stirring the reaction mixture at $25{ }^{\circ} \mathrm{C}$ for $72 \mathrm{~h}$ followed by flash column chromatography (Pet. ether-EtOAc: 85:15) to afford $(S)-6-(2-$ bromophenyl)-2-phenyl-5,6-dihydro-4H-1,3-thiazin-4-one $\mathbf{3 m}$ as a yellow oil (78 $\mathrm{mg}, 90 \%$ yield). $\boldsymbol{R}_{f}($ Pet. ether $/ \mathrm{EtOAc}=70 / 30): 0.65 ; \mathrm{er}=91: 9,[\alpha]_{\mathrm{D}}{ }^{25}=-13.3\left(\mathrm{c} 0.1, \mathrm{CHCl}_{3}\right)$. HPLC $($ ChiralpakIF, 80:20 Hexane / $i$-PrOH, $0.7 \mathrm{~mL} / \mathrm{min}, 254 \mathrm{~nm}$ ) Minor: $25.4 \mathrm{~min}$, Major: 28.7 min. ${ }^{1}$ H NMR (400 MHz, CDCl $) \delta$ 8.08-8.06 (m, 2H), 7.61-7.55 (m, 2H), 7.45-7.41 (m, 3H), 7.35-7.32 (m, 1H), 7.21-7.17 (m, 1H), $5.34\left(\mathrm{dd}, J_{1}=9.5 \mathrm{~Hz}, J_{2}=5.4 \mathrm{~Hz}, 1 \mathrm{H}\right), 3.05-2.94(\mathrm{~m}, 2 \mathrm{H}) .{ }^{13} \mathbf{C}$ NMR (100 MHz, $\left.\mathbf{C D C l}_{3}\right) \delta 178.91,178.37,136.32,133.74,130.29,128.79,128.48,127.76,123.92,43.74$, 35.41. HRMS (ESI) calculated [M+H] for $\mathrm{C}_{16} \mathrm{H}_{13} \mathrm{BrNOS}: 345.9901$, found: 345.9900 . FTIR (cm1) $2922,2851,1713,1606,1509,1472,1376,1308,1243,1194,1024,898$.

\section{(S)-6-(2-Chlorophenyl)-2-phenyl-5,6-dihydro-4H-1,3-thiazin-4-one (3n)}<smiles>O=C1CC(c2ccccc2Cl)SC(c2ccccc2)=N1</smiles>

$3 n$

Following the general procedure, treatment of (Z)-2-bromo-3-(2chlorophenyl)acrylaldehyde $\quad \mathbf{2 n} \quad\left(\begin{array}{llll}72.3 & \mathrm{mg}, & 0.30 & \mathrm{mmol})\end{array}\right.$ and benzothioamide 1a (34.3 mg, $0.25 \mathrm{mmol})$ with triazolium salt $\mathbf{C}(11.6 \mathrm{mg}$, $0.025 \mathrm{mmol})$, lithium acetate dihydrate $(5.1 \mathrm{mg}, 0.05 \mathrm{mmol}), \mathrm{K}_{2} \mathrm{CO}_{3}(52.0$ $\mathrm{mg}, 0.375 \mathrm{mmol})$ and $4 \AA \mathrm{MS}(100 \mathrm{mg})$ in mesitylene $(2.0 \mathrm{~mL})$ and stirring the reaction mixture at $25{ }^{\circ} \mathrm{C}$ for $72 \mathrm{~h}$ followed by flash column chromatography (Pet. ether-EtOAc: $85: 15$ ) to afford (S)-6-(2-chlorophenyl)-2-phenyl-5,6-dihydro-4H-1,3-thiazin-4-one 3n as a pale yellow sticky liquid (53 mg, 70\% yield).

$\boldsymbol{R}_{f}($ Pet. ether $/ \mathrm{EtOAc}=70 / 30): 0.62 ; \mathrm{er}=92: 8,[\alpha]_{\mathrm{D}}{ }^{25}=-73.6\left(\mathrm{c} 0.1, \mathrm{CHCl}_{3}\right) . \mathbf{H P L C}($ ChiralpakIC, 70:30 Hexane / $i$-PrOH, $1.0 \mathrm{~mL} / \mathrm{min}, 254 \mathrm{~nm}$ ) Minor: $23.2 \mathrm{~min}$, Major: $21.8 \mathrm{~min} .{ }^{1} \mathbf{H}$ NMR (400 MHz, $\left.\mathbf{C D C l}_{3}\right) \delta 8.09$ (d, $\left.J=7.6 \mathrm{~Hz}, 2 \mathrm{H}\right), 7.59(\mathrm{t}, J=7.2 \mathrm{~Hz}, 1 \mathrm{H}), 7.48-7.42(\mathrm{~m}, 4 \mathrm{H}), 7.32-7.29$ $(\mathrm{m}, 2 \mathrm{H}), 5.38\left(\mathrm{dd}, J_{1}=9.1 \mathrm{~Hz}, J_{2}=5.8 \mathrm{~Hz}, 1 \mathrm{H}\right), 3.06-2.97(\mathrm{~m}, 2 \mathrm{H}) \cdot{ }^{\mathbf{1 3}} \mathbf{C} \mathbf{N M R}\left(\mathbf{1 0 0} \mathbf{M H z}, \mathbf{C D C l}_{\mathbf{3}}\right)$ $\delta 179.08,178.50,136.43,134.81,133.97,133.60,130.43,130.09,128.93,128.81,128.60,128.07$, 


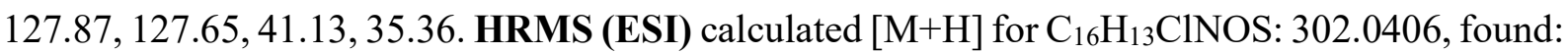
302.0404. FTIR (cm $\left.{ }^{-1}\right)$ 2959, 2923, 1714, 1677, 1573, 1509, 1474, 1375, 1243, 1153, 1035, 908.

\section{(S)-6-(3,4-Dichlorophenyl)-2-phenyl-5,6-dihydro-4H-1,3-thiazin-4-one (3o)}<smiles>O=C1CC(c2ccc(Cl)c(Cl)c2)SC(c2ccccc2)=N1</smiles>

Following the general procedure, treatment of (Z)-2-bromo-3-(3,4dichlorophenyl)acrylaldehyde $\quad \mathbf{2 0} \quad\left(\begin{array}{lllll}83.9 & \mathrm{mg}, & 0.30 & \mathrm{mmol}\end{array}\right)$ andbenzothioamide 1a $(34.3 \mathrm{mg}, 0.25 \mathrm{mmol})$ with triazolium salt $\mathbf{C}$ (11.6 mg, $0.025 \mathrm{mmol})$,lithium acetate dihydrate $(5.1 \mathrm{mg}, 0.05 \mathrm{mmol}$ ), $\mathrm{K}_{2} \mathrm{CO}_{3}(52.0 \mathrm{mg}, 0.375 \mathrm{mmol})$ and $4 \AA \mathrm{MS}(100 \mathrm{mg})$ in mesitylene $(2.0 \mathrm{~mL})$ and stirring the reaction mixture at $25^{\circ} \mathrm{C}$ for $72 \mathrm{~h}$ followed by flash column chromatography (Pet. ether-EtOAc: 85:15) to afford (S)-6-(3,4-dichlorophenyl)-2-phenyl-5,6-dihydro-4H-1,3-thiazin-4-one 3o as a pale yellow solid (30 mg, 36\% yield).

$\boldsymbol{R}_{\boldsymbol{f}}($ Pet. ether $/ \mathrm{EtOAc}=70 / 30): 0.61$. er $=92: 8,[\alpha]_{\mathrm{D}}{ }^{25}=+50.2\left(\mathrm{c} 0.1, \mathrm{CHCl}_{3}\right)$. HPLC $($ ChiralpakIF, 80:20 Hexane / $i$-PrOH, $0.7 \mathrm{~mL} / \mathrm{min}, 254 \mathrm{~nm}$ ) Minor: 24.9 min, Major: 26.5 min. ${ }^{1}$ H NMR (400 MHz, $\left.\mathbf{C D C l}_{3}\right) \delta 8.08(\mathrm{~d}, J=8.2 \mathrm{~Hz}, 2 \mathrm{H}), 7.61$ (t, $\left.J=7.3 \mathrm{~Hz} 1 \mathrm{H}\right), 7.50-7.45$ (m, 4H), 7.24-7.21(m, $1 \mathrm{H}), 4.80\left(\mathrm{dd}, J_{1}=12.2 \mathrm{~Hz}, J_{2}=4.1 \mathrm{~Hz}, 1 \mathrm{H}\right), 3.04\left(\mathrm{dd}, J_{1}=14.1 \mathrm{~Hz}, J_{2}=4.2 \mathrm{~Hz}, 1 \mathrm{H}\right), 2.95-2.88$ $(\mathrm{m}, 1 \mathrm{H}) .{ }^{13} \mathbf{C}$ NMR (100 MHz, $\left.\mathbf{C D C l}_{3}\right) \delta 178.45,178.37,137.38,136.15,134.07,133.65,133.43$, 131.47, 129.74, 128.97, 127.90, 126.96, 44.00, 36.75. HRMS (ESI) calculated [M+H] for $\mathrm{C}_{16} \mathrm{H}_{12} \mathrm{Cl}_{2} \mathrm{NOS}: 336.0017$, found: 336.0000. FTIR (cm $\left.{ }^{-1}\right)$ 2959, 2922, 1682, 1519, 1472, 1399, 1374, 1243, 1213, 1131, 1030, 944.

\section{(S)-6-(3-Bromo-4-methoxyphenyl)-2-phenyl-5,6-dihydro-4H-1,3-thiazin-4-one (3p)}

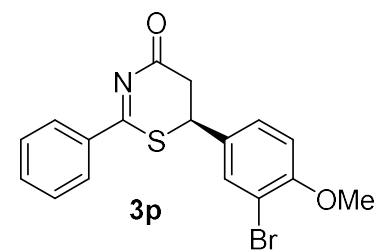

Following the general procedure, treatment of (Z)-2-bromo-3-(3-bromo4-methoxyphenyl)acrylaldehyde $\quad \mathbf{2 p} \quad(96 \quad \mathrm{mg}, \quad 0.30 \quad \mathrm{mmol})$ and benzothioamide 1a (34.3 mg, $0.25 \mathrm{mmol})$ with triazolium salt $\mathbf{C}(11.6 \mathrm{mg}$, $0.025 \mathrm{mmol})$, lithium acetate dihydrate $(5.1 \mathrm{mg}, 0.05 \mathrm{mmol}), \mathrm{K}_{2} \mathrm{CO}_{3}(52.0$ $\mathrm{mg}, 0.375 \mathrm{mmol})$ and $4 \AA \mathrm{MS}(100 \mathrm{mg})$ in mesitylene $(2.0 \mathrm{~mL})$ and stirring the reaction mixture at $25{ }^{\circ} \mathrm{C}$ for $72 \mathrm{~h}$ followed by flash column chromatography (Pet. ether-EtOAc: $85: 15$ ) to afford (S)-6-(3-Bromo-4-methoxyphenyl)-2-phenyl-5,6-dihydro-4H-1,3-thiazin-4-one 3p as a yellow solid(45 mg, 48\% yield). 
$\boldsymbol{R}_{\boldsymbol{f}}($ Pet. ether $/$ EtOAc $=70 / 30): 0.56 ; \mathrm{er}=90: 10,[\alpha]_{\mathrm{D}}{ }^{20}=-82.0\left(\mathrm{c} 0.05, \mathrm{CHCl}_{3}\right)$. HPLC $($ Chiralpak IC, 70:30 Hexane / $i$-PrOH, $1.0 \mathrm{~mL} / \mathrm{min}, 254 \mathrm{~nm}$ ) Minor: $42.4 \mathrm{~min}$, Major: 33.6 min. ${ }^{1} \mathbf{H}$ NMR (400 MHz, $\left.\mathbf{C D C l}_{3}\right) \delta$ 8.08-8.07 (m, 2H), 7.59-7.57 (m, 2H), $7.46(\mathrm{t}, J=7.8 \mathrm{~Hz}, 2 \mathrm{H}), 7.30-7.27(\mathrm{~m}$, $1 \mathrm{H}), 6.91(\mathrm{~d}, J=8.6 \mathrm{~Hz}, 1 \mathrm{H}), 4.76\left(\mathrm{dd}, J_{1}=12.7 \mathrm{~Hz}, J_{2}=4.0 \mathrm{~Hz}, 1 \mathrm{H}\right), 3.91(\mathrm{~s}, 3 \mathrm{H}), 3.03\left(\mathrm{dd}, J_{1}=\right.$ $\left.14.0 \mathrm{~Hz}, J_{2}=4.0 \mathrm{~Hz}, 1 \mathrm{H}\right), 2.92(\mathrm{~m}, 1 \mathrm{H}) .{ }^{13} \mathbf{C} \mathbf{~ N M R}\left(\mathbf{1 0 0} \mathbf{~ M H z}, \mathbf{C D C l}_{3}\right) \delta 179.12,179.10,156.44$, $136.31,133.99,132.60,132.46,130.35,128.91,127.91,112.48,112.34,44.07,37.16 . . H R M S$ (ESI) calculated $[\mathrm{M}+\mathrm{H}]$ for $\mathrm{C}_{17} \mathrm{H}_{15} \mathrm{BrNO}_{2} \mathrm{~S}: 376.0007$, found: 376.0007. FTIR (cm $\left.{ }^{-1}\right)$ 2962, 2925, 2842, 1714, 1663, 1601, 1498, 1370, 1255, 1054, 1018, 909.

(S)-6-(2-Bromo-4,5-dimethoxyphenyl)-2-phenyl-5,6-dihydro-4H-1,3-thiazin-4-one (3q)

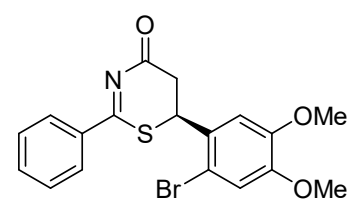

$3 q$

Following the general procedure, treatment of (Z)-2-bromo-3-(2-bromo4,5-dimethoxyphenyl)acrylaldehyde $\mathbf{2 q}(81.3 \mathrm{mg}, 0.30 \mathrm{mmol})$ and benzothioamide 1a $(34.3 \mathrm{mg}, 0.25 \mathrm{mmol})$ with triazolium salt $\mathbf{C}(11.6 \mathrm{mg}$, $0.025 \mathrm{mmol})$, lithium acetate dihydrate $(5.1 \mathrm{mg}, 0.05 \mathrm{mmol}), \mathrm{K}_{2} \mathrm{CO}_{3}(52.0$ $\mathrm{mg}, 0.375 \mathrm{mmol})$ and $4 \AA \mathrm{MS}(100 \mathrm{mg})$ in mesitylene $(2.0 \mathrm{~mL})$ and stirring the reaction mixture at $25{ }^{\circ} \mathrm{C}$ for $72 \mathrm{~h}$ followed by flash column chromatography (Pet. ether-EtOAc: 80:20) to afford (S)-6-(2-bromo-4,5-dimethoxyphenyl)-2-phenyl-5,6-dihydro-4H-1,3-thiazin-4-one $\mathbf{3 q}$ as a pale yellow solid (48 $\mathrm{mg}, 47 \%$ yield).

$\boldsymbol{R}_{\boldsymbol{f}}($ Pet. ether $/$ EtOAc $=70 / 30): 0.5$; er $=82: 18,[\alpha]_{\mathrm{D}}{ }^{25}=-11.5\left(\mathrm{c} 0.1, \mathrm{CHCl}_{3}\right)$. HPLC (Chiralpak IF, 70:30 Hexane / $i$-PrOH, $0.7 \mathrm{~mL} / \mathrm{min}, 254 \mathrm{~nm}$ ) Minor: $25.3 \mathrm{~min}$, Major: $51.1 \mathrm{~min} .{ }^{1} \mathbf{H}$ NMR (400 MHz, $\left.\mathbf{C D C l}_{3}\right) \delta$ 8.10-8.07 (m, 2H), 7.61-7.57 (m, 1H), 7.48-7.44 (m, 2H), $7.05(\mathrm{~s}, 1 \mathrm{H}), 6.93$ $(\mathrm{s}, 1 \mathrm{H}), 5.30\left(\mathrm{dd}, J_{1}=9.8 \mathrm{~Hz}, J_{2}=5.2 \mathrm{~Hz}, 1 \mathrm{H}\right), 3.88(\mathrm{~s}, 3 \mathrm{H}), 3.84(\mathrm{~s}, 3 \mathrm{H}), 3.05-2.94(\mathrm{~m}, 2 \mathrm{H}),{ }^{13} \mathrm{C}$ NMR (100 MHz, $\left.\mathbf{C D C l}_{3}\right) \delta$ 179.25, 178.82, 149.88, 149.16, 136.44, 133.90, 128.87, 128.10, 127.86, 115.93, 114.20, 110.68, 56.39, 56.35, 43.88, 35.83. HRMS (ESI) calculated [M+H] for $\mathrm{C}_{18} \mathrm{H}_{18} \mathrm{BrNO}_{3} \mathrm{~S}:$ 406.0113, found: 406.0124. FTIR (cm $\left.{ }^{-1}\right)$ 2962, 2925, 2845, 1737, 1714, 1660, 1600, 1506, 1465, 1439, 1381, 1261, 1162, 1026.

\section{(S)-6-(Furan-2-yl)-2-phenyl-5,6-dihydro-4H-1,3-thiazin-4-one (3r)}

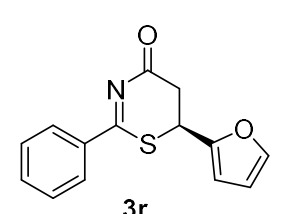

$3 r$

Following the general procedure, treatment of (Z)-2-bromo-3-(furan-2yl)acrylaldehyde $\mathbf{2 a}(60.3 \mathrm{mg}, 0.30 \mathrm{mmol})$ and benzothioamide $\mathbf{1 a}(34.3 \mathrm{mg}$, $0.25 \mathrm{mmol})$ with triazolium salt $\mathbf{C}(11.6 \mathrm{mg}, 0.025 \mathrm{mmol})$, lithium acetate 
dihydrate (5.1 mg, $0.05 \mathrm{mmol}), \mathrm{K}_{2} \mathrm{CO}_{3}(52.0 \mathrm{mg}, 0.375 \mathrm{mmol})$ and $4 \AA \mathrm{MS}(100 \mathrm{mg})$ in mesitylene $(2.0 \mathrm{~mL})$ and stirring the reaction mixture at $25{ }^{\circ} \mathrm{C}$ for $72 \mathrm{~h}$ followed by flash column chromatography (Pet. ether-EtOAc: 85:15) to afford (S)-6-(Furan-2-yl)-2-phenyl-5,6-dihydro-4H1,3-thiazin-4-one $3 \mathbf{r}$ as a pale yellow solid ( $28 \mathrm{mg}, 41 \%$ yield).

$\boldsymbol{R}_{\boldsymbol{f}}($ Pet. ether $/ \mathrm{EtOAc}=80 / 20): 0.48$; er $=89: 11,[\alpha]_{\mathrm{D}}{ }^{25}=-136.9\left(\mathrm{c} 0.1, \mathrm{CHCl}_{3}\right)$. HPLC $($ Chiralpak IC, 70:30 Hexane / i-PrOH, $1.0 \mathrm{~mL} / \mathrm{min}, 254 \mathrm{~nm}$ ) Minor: $22.3 \mathrm{~min}$, Major: $23.9 \mathrm{~min} .{ }^{1} \mathbf{H}$ NMR $\left(400 \mathbf{M H z}, \mathbf{C D C l}_{3}\right) \delta 8.07$ (d, $\left.J=7.9 \mathrm{~Hz}, 2 \mathrm{H}\right), 7.59$ (t, $\left.J=7.1 \mathrm{~Hz}, 1 \mathrm{H}\right), 7.47-7.43$ (m, 3H), 6.36 $(\mathrm{s}, 2 \mathrm{H}), 4.95\left(\mathrm{dd}, J_{1}=9.0 \mathrm{~Hz}, J_{2}=5.3 \mathrm{~Hz}, 1 \mathrm{H}\right), 3.15-3.04(\mathrm{~m}, 2 \mathrm{H}) .{ }^{13} \mathbf{C}$ NMR (100 MHz, CDCl $\left.\mathbf{3}\right)$ $\delta 178.59,178.07,150.02,143.57,136.54,133.86,132.08,128.88,128.59,127.88,127.06,110.95$, 108.53, 38.06, 34.00. HRMS (ESI) calculated [M+H] for $\mathrm{C}_{14} \mathrm{H}_{12} \mathrm{NO}_{2} \mathrm{~S}$ : 258.0589, found: 258.0591. FTIR (cm-1) 2959, 2921, 1687, 1624, 1516, 1449, 1379, 1327, 1222, 1128.

\section{(S)-2-Phenyl-6-(thiophen-2-yl)-5,6-dihydro-4H-1,3-thiazin-4-one (3s)}

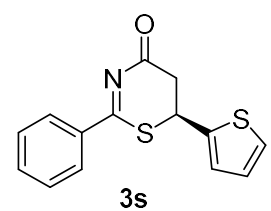

Following the general procedure, treatment of (Z)-2-bromo-3-(thiophen-2yl)acrylaldehyde $2 \mathrm{~s}(65.1 \mathrm{mg}, 0.30 \mathrm{mmol})$ and benzothioamide $1 \mathrm{a}(34.3 \mathrm{mg}$, $0.25 \mathrm{mmol})$ with triazolium salt $\mathbf{C}(11.6 \mathrm{mg}, 0.025 \mathrm{mmol})$, lithium acetate dihydrate (5.1 mg, $0.05 \mathrm{mmol}), \mathrm{K}_{2} \mathrm{CO}_{3}(52.0 \mathrm{mg}, 0.375 \mathrm{mmol})$ and $4 \AA \mathrm{MS}(100$ $\mathrm{mg})$ in mesitylene $(2.0 \mathrm{~mL})$ and stirring the reaction mixture at $25^{\circ} \mathrm{C}$ for $72 \mathrm{~h}$ followed by flash column chromatography (Pet. ether-EtOAc: 85:15) to afford (S)-2-phenyl-6-(thiophen-2-yl)-5,6dihydro-4H-1,3-thiazin-4-one 3s as a brown oil (44 mg, 64\% yield).

$\boldsymbol{R}_{\boldsymbol{f}}($ Pet. ether $/ \mathrm{EtOAc}=70 / 30): 0.5$; er $=91: 9,[\alpha]_{\mathrm{D}}{ }^{20}=-116.0\left(\mathrm{c} 0.1, \mathrm{CHCl}_{3}\right)$. HPLC (Chiralpak IC, 70:30 Hexane / $i$-PrOH, $1.0 \mathrm{~mL} / \mathrm{min}, 254 \mathrm{~nm}$ ) Minor: $29.1 \mathrm{~min}$, Major: $26.8 \mathrm{~min} .{ }^{1} \mathbf{H}$ NMR (400 MHz, $\left.\mathbf{C D C l}_{3}\right) \delta$ 8.09-8.06 (m, 2H), 7.61-7.57 (m, 1H), 7.48-7.44 (m, 2H), 7.43-7.32 (m, 1H), $7.10(\mathrm{~d}, J=3.5 \mathrm{~Hz}, 1 \mathrm{H}), 7.00\left(\mathrm{dd}, J_{1}=5.1 \mathrm{~Hz}, J_{2}=3.6 \mathrm{~Hz}, 1 \mathrm{H}\right), 5.13\left(\mathrm{dd}, J_{1}=11.9 \mathrm{~Hz}, J_{2}=4.0\right.$ $\mathrm{Hz}, 1 \mathrm{H}), 3.17\left(\mathrm{dd}, J_{1}=14.3 \mathrm{~Hz}, J_{2}=4.0 \mathrm{~Hz}, 1 \mathrm{H}\right), 3.01\left(\mathrm{dd}, J_{1}=14.3 \mathrm{~Hz}, J_{2}=11.9 \mathrm{~Hz}, 1 \mathrm{H}\right) .{ }^{13} \mathbf{C}$ NMR (100 MHz, $\left.\mathbf{C D C l}_{3}\right) \delta$ 178.77, 178.46, 140.44, 136.37, 133.89, 128.89, 127.93, 127.50, 126.57, 126.48, 40.51, 38.14. HRMS (ESI) calculated [M+H] for $\mathrm{C}_{14} \mathrm{H}_{12} \mathrm{NOS}_{2}: 274.0360$, found: 274.0364. FTIR (cm (c) $^{-1}$ 3064, 2960, 2923, 2852, 1714, 1678, 1605, 1508, 1475, 1373, 1245, 1105 , 1025. 
(R)-6-Methyl-2-phenyl-5,6-dihydro-4H-1,3-thiazin-4-one (3t)

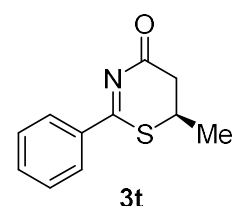

$3 t$

Following the general procedure, treatment of (Z)-2-bromobut-2-enal $2 \mathbf{t}(44.7 \mathrm{mg}$, $0.30 \mathrm{mmol})$ and benzothioamide $\mathbf{1 a}(34.3 \mathrm{mg}, 0.25 \mathrm{mmol})$ with triazolium salt $\mathbf{C}$

(11.6 mg, $0.025 \mathrm{mmol})$, lithium acetate dihydrate $(5.1 \mathrm{mg}, 0.05 \mathrm{mmol}), \mathrm{K}_{2} \mathrm{CO}_{3}$ $(52.0 \mathrm{mg}, 0.375 \mathrm{mmol})$ and $4 \AA \mathrm{MS}(100 \mathrm{mg})$ in mesitylene $(2.0 \mathrm{~mL})$ and stirring the reaction mixture at $25{ }^{\circ} \mathrm{C}$ for $72 \mathrm{~h}$ followed by flash column chromatography (Pet. etherEtOAc: $85: 15)$ to afford $(R)-6$-methyl-2-phenyl-5,6-dihydro-4H-1,3-thiazin-4-one $3 \mathrm{t}$ as a yellow oil (28 mg, $54 \%$ yield).

$\boldsymbol{R}_{\boldsymbol{f}}($ Pet. ether $/$ EtOAc $=80 / 20): 0.41 ; \mathrm{er}=88: 12,[\alpha]_{\mathrm{D}}{ }^{25}=+13.0\left(\mathrm{c} 0.1, \mathrm{CHCl}_{3}\right)$. HPLC $($ Chiralpak IF, 80:20 Hexane / $i$-PrOH, $0.7 \mathrm{~mL} / \mathrm{min}, 254 \mathrm{~nm}$ ) Minor: $18.9 \mathrm{~min}$, Major: $20.5 \mathrm{~min} .{ }^{1} \mathbf{H}$ NMR (400 MHz, $\left.\mathbf{C D C l}_{3}\right) \delta$ 8.05-8.03 (m, 2H), 7.58-7.54 (m, 1H), 7.45-7.41 (m, 2H), 3.79-3.70 (m, 1H), $2.84\left(\mathrm{dd}, J_{1}=14.0 \mathrm{~Hz}, J_{2}=4.2 \mathrm{~Hz}, 1 \mathrm{H}\right), 2.51\left(\mathrm{dd}, J_{1}=14.2 \mathrm{~Hz}, J_{2}=10.4 \mathrm{~Hz}, 1 \mathrm{H}\right) 1.49(\mathrm{~d}, J=7.1$ $\mathrm{Hz}, 3 \mathrm{H}) .{ }^{13} \mathbf{C}$ NMR (100 MHz, $\left.\mathbf{C D C l}_{3}\right) \delta$ 179.05, 178.95, 136.67, 133.55, 128.75, 127.75, 37.73, 36.08, 21.05. HRMS (ESI) calculated [M+H] for $\mathrm{C}_{11} \mathrm{H}_{12} \mathrm{NOS}: 206.0640$, found: 206.0637. FTIR (cm-1) 3063, 3028, 2922, 2852, 1713, 1657, 1484, 1378, 1245, 1149, 1077, 905.

\section{(R)-6-Ethyl-2-phenyl-5,6-dihydro-4H-1,3-thiazin-4-one (3u)}

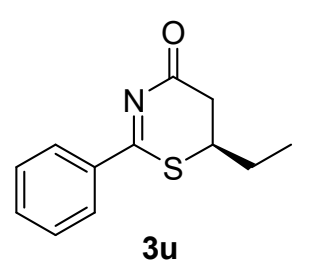

Following the general procedure, treatment of (Z)-2-bromopent-2-enal $\mathbf{2 u}$ $(48.9 \mathrm{mg}, 0.30 \mathrm{mmol})$ and benzothioamide $1 \mathrm{a}(34.3 \mathrm{mg}, 0.25 \mathrm{mmol})$ with triazolium salt $\mathbf{C}(11.6 \mathrm{mg}, 0.025 \mathrm{mmol})$, lithium acetate dihydrate $(5.1 \mathrm{mg}$, $0.05 \mathrm{mmol}), \mathrm{K}_{2} \mathrm{CO}_{3}(52.0 \mathrm{mg}, 0.375 \mathrm{mmol})$ and $4 \AA \mathrm{MS}(100 \mathrm{mg})$ in mesitylene $(2.0 \mathrm{~mL})$ and stirring the reaction mixture at $25{ }^{\circ} \mathrm{C}$ for $72 \mathrm{~h}$ followed by flash column chromatography (Pet. ether-EtOAc: 85:15) to afford (R)-6-ethyl-2phenyl-5,6-dihydro-4H-1,3-thiazin-4-one $3 \mathbf{u}$ as a pale yellow liquid (41 $\mathrm{mg}, 75 \%$ yield).

$\boldsymbol{R}_{f}($ Pet. ether $/$ EtOAc $=70 / 30): 0.65$; er $=89: 11,[\alpha]_{\mathrm{D}^{20}}=-50.0\left(\mathrm{c} 0.05, \mathrm{CHCl}_{3}\right)$. HPLC $($ Chiralcel OJ-H, 85:15 Hexane / $i$-PrOH, $1.0 \mathrm{~mL} / \mathrm{min}, 254 \mathrm{~nm}$ ) Minor: $16.2 \mathrm{~min}$, Major: $19.6 \mathrm{~min} .{ }^{1}$ H NMR (400 MHz, $\left.\mathbf{C D C l}_{3}\right) \delta$ 8.06-8.04 (m, 2H), 7.57-7.53 (m, 1H), 7.45-7.41 (m, 2H), 3.74-3.55 (m, 1H), $2.84\left(\mathrm{dd}, J_{1}=14.0 \mathrm{~Hz}, J_{2}=4.4 \mathrm{~Hz}, 1 \mathrm{H}\right), 2.58\left(\mathrm{dd}, J_{1}=14.0 \mathrm{~Hz}, J_{2}=10.1 \mathrm{~Hz}, 1 \mathrm{H}\right), 1.95-1.72(\mathrm{~m}$, 2H), $1.08(\mathrm{t}, J=7.3 \mathrm{~Hz}, 3 \mathrm{H}) .{ }^{13} \mathbf{C}$ NMR (100 MHz, $\left.\mathbf{C D C l}_{3}\right) \delta 179.18,178.88,136.78,133.50$, 128.73, 127.74, 42.90, 35.85, 28.46, 11.01. HRMS (ESI) calculated [M+H] for $\mathrm{C}_{13} \mathrm{H}_{14} \mathrm{NOS}$ : 
220.0796, found: 220.0800. FTIR (cm $\left.{ }^{-1}\right)$ 2967, 2927, 1712, 1679, 1574, 1511, 1475, 1378, 1240, $1155,1073,910$.

\section{(S)-2-(4-Methoxyphenyl)-6-phenyl-5,6-dihydro-4H-1,3-thiazin-4-one (3v)}<smiles>COc1ccc(C2=NC(=O)CC(c3ccccc3)S2)cc1</smiles>

$3 v$

Following the general procedure, treatment of (Z)-2-bromo-3phenylacrylaldehyde $\mathbf{1 a} \quad\left(\begin{array}{llll}63.4 & \mathrm{mg}, & 0.30 & \mathrm{mmol})\end{array}\right)$ and 4methoxybenzothioamide $2 \mathbf{v}(41.8 \mathrm{mg}, 0.25 \mathrm{mmol})$ with triazolium salt $\mathbf{C}$ (11.6 mg, $0.025 \mathrm{mmol})$, lithium acetate dihydrate $(5.1 \mathrm{mg}, 0.05 \mathrm{mmol})$, $\mathrm{K}_{2} \mathrm{CO}_{3}(52.0 \mathrm{mg}, 0.375 \mathrm{mmol})$ and $4 \AA \mathrm{MS}(100 \mathrm{mg})$ in mesitylene $(2.0 \mathrm{~mL})$ and stirring the reaction mixture at $25^{\circ} \mathrm{C}$ for $72 \mathrm{~h}$ followed by flash column chromatography (Pet. ether-EtOAc: 80:20) to afford (S)-2-(4-methoxyphenyl)-6-phenyl-5,6-dihydro-4H-1,3-thiazin-4-one 3v as a pale yellow solid (41 mg, 55\% yield).

$\boldsymbol{R}_{\boldsymbol{f}}($ Pet. ether $/ \mathrm{EtOAc}=70 / 30): 0.54$; er $=92: 8,[\alpha]_{\mathrm{D}}^{22}=-60.7\left(\mathrm{c} 0.1, \mathrm{CHCl}_{3}\right)$. HPLC $($ Chiralpak IF, 75:25 Hexane / $i$-PrOH, $0.7 \mathrm{~mL} / \mathrm{min}, 254 \mathrm{~nm}$ ) Minor: $36.8 \mathrm{~min}$, Major: $44.0 \mathrm{~min} .{ }^{1} \mathbf{H}$ NMR (400 MHz, $\left.\mathbf{C D C l}_{3}\right) \delta$ 8.11-8.09 (m, 2H), 7.43-7.35 (m, 5H), 6.95-6.93 (m, 2H), $4.80\left(\mathrm{dd}, J_{1}=12.6\right.$ $\left.\mathrm{Hz}, J_{2}=3.9 \mathrm{~Hz}, 1 \mathrm{H}\right), 3.88(\mathrm{~s}, 3 \mathrm{H}), 3.06\left(\mathrm{dd}, J_{l}=14.3 \mathrm{~Hz}, J_{2}=4.0 \mathrm{~Hz}, 1 \mathrm{H}\right), 2.95\left(\mathrm{dd}, J_{l}=14.4 \mathrm{~Hz}\right.$, $\left.J_{2}=12.8 \mathrm{~Hz}, 1 \mathrm{H}\right) .{ }^{13} \mathbf{C} \mathbf{N M R}\left(\mathbf{1 0 0} \mathbf{~ M H z}, \mathbf{C D C l}_{3}\right) \delta 179.38,178.43,164.49,137.31,130.09,129.46$, 129.07, 128.88, 127.67, 114.20, 55.73, 37.31. HRMS (ESI) calculated [M+H] for $\mathrm{C}_{17} \mathrm{H}_{16} \mathrm{NO}_{2} \mathrm{~S}$ : 298.0902, found: 298.0902. FTIR (cm-1) 2962, 2843, 1711, 1673, 1603, 1488, 1377, 1312, 1251, $1175,1025,910,844$.

\section{(S)-6-Phenyl-2-(p-tolyl)-5,6-dihydro-4H-1,3-thiazin-4-one (3w)}

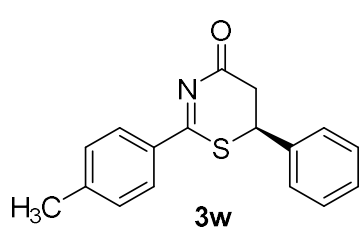

$3 w$

Following the general procedure, treatment of (Z)-2-bromo-3phenylacrylaldehyde $\quad 2 s \quad\left(\begin{array}{llll}63.4 & \mathrm{mg}, & 0.30 & \mathrm{mmol}\end{array}\right)$ and 4methylbenzothioamide $\mathbf{1 w}(37.8 \mathrm{mg}, 0.25 \mathrm{mmol})$ with triazolium salt $\mathbf{C}$ (11.6 $\mathrm{mg}, 0.025 \mathrm{mmol})$, lithium acetate dihydrate $(5.1 \mathrm{mg}, 0.05 \mathrm{mmol})$, $\mathrm{K}_{2} \mathrm{CO}_{3}(52.0 \mathrm{mg}, 0.375 \mathrm{mmol})$ and $4 \AA \mathrm{MS}(100 \mathrm{mg})$ in mesitylene $(2.0 \mathrm{~mL})$ and stirring the reaction mixture at $25^{\circ} \mathrm{C}$ for $72 \mathrm{~h}$ followed by flash column chromatography (Pet. ether-EtOAc: 85:15) to afford(S)-6-phenyl-2-(p-tolyl)-5,6-dihydro-4H-1,3-thiazin-4-one $\mathbf{3 w}$ as a pale yellow solid (40 mg, 57\% yield). 
$\boldsymbol{R}_{\boldsymbol{f}}($ Pet. ether $/ \mathrm{EtOAc}=70 / 30): 0.64 ; \mathrm{er}=93: 7,[\alpha]_{\mathrm{D}}{ }^{25}=-73.8\left(\mathrm{c} 0.1, \mathrm{CHCl}_{3}\right) . \mathbf{H P L C}($ ChiralpakIC, 70:30 Hexane / $i$-PrOH, $1.0 \mathrm{~mL} / \mathrm{min}, 254 \mathrm{~nm}$ ) Minor: $37.2 \mathrm{~min}$, Major: 33.9 min. ${ }^{1}$ H NMR (400 MHz, CDCl$)_{3} \delta 7.99\left(\mathrm{~d}, J_{1}=8.2 \mathrm{~Hz}, 2 \mathrm{H}\right), 7.42-7.38(\mathrm{~m}, 5 \mathrm{H}), 7.27\left(\mathrm{~d}, J_{1}=7.9 \mathrm{~Hz}, 2 \mathrm{H}\right), 4.82(\mathrm{dd}$, $\left.J_{1}=12.7 \mathrm{~Hz}, J_{2}=4.1 \mathrm{~Hz}, 1 \mathrm{H}\right), 3.07\left(\mathrm{dd}, J_{1}=14.2 \mathrm{~Hz}, J_{2}=4.2 \mathrm{~Hz}, 1 \mathrm{H}\right), 3.01-2.94(\mathrm{~m}, 1 \mathrm{H}), 2.43$ (s, 3H). ${ }^{13} \mathbf{C}$ NMR (100 MHz, $\left.\mathbf{C D C l}_{3}\right) \delta 179.39,179.20,144.92,137.18,133.74,129.58,127.96$, 127.67, 44.99, 37.14, 21.82. HRMS (ESI) calculated [M+H] for $\mathrm{C}_{17} \mathrm{H}_{16} \mathrm{NOS}$ : 282.0953, found: 282.0954. FTIR (cm-1) 2958, 2921, 2853, 1713, 1659, 1609, 1488, 1376, 1248, 1185, 1083, 910.

\section{(S)-2-(4-Iodophenyl)-6-phenyl-5,6-dihydro-4H-1,3-thiazin-4-one (3x)}

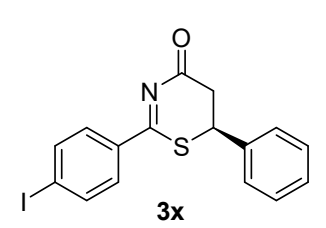

Following the general procedure, treatment of (Z)-2-bromo-3phenylacrylaldehyde $\mathbf{2 a}(63.4 \mathrm{mg}, 0.30 \mathrm{mmol})$ and 4-iodobenzothioamide $\mathbf{1 x}$ (65.8 mg, $0.25 \mathrm{mmol})$ with triazolium salt $\mathbf{C}(11.6 \mathrm{mg}, 0.025 \mathrm{mmol})$, lithium acetate dihydrate $(5.1 \mathrm{mg}, 0.05 \mathrm{mmol}), \mathrm{K}_{2} \mathrm{CO}_{3}(52.0 \mathrm{mg}, 0.375 \mathrm{mmol})$ and 4 $\AA \mathrm{MS}(100 \mathrm{mg})$ in mesitylene $(2.0 \mathrm{~mL})$ and stirring the reaction mixture at $25^{\circ} \mathrm{C}$ for $72 \mathrm{~h}$ followed by flash column chromatography (Pet. ether-EtOAc: 80:20) to afford (S)-2-(4-iodophenyl)-6phenyl-5,6-dihydro-4H-1,3-thiazin-4-one $\mathbf{3 x}$ as a pale yellow solid (68 $\mathrm{mg}, 69 \%$ yield).

$\boldsymbol{R}_{\boldsymbol{f}}($ Pet. ether $/ \mathrm{EtOAc}=70 / 30): 0.56 ; \mathrm{er}=94: 6,[\alpha]_{\mathrm{D}}{ }^{25}=+191.24\left(\mathrm{c} 0.1, \mathrm{CHCl}_{3}\right)$. HPLC (Chiralpak IF, 80:20 Hexane / $i$-PrOH, $0.7 \mathrm{~mL} / \mathrm{min}, 254 \mathrm{~nm}$ ) Minor: $36.3 \mathrm{~min}$, Major: $38.7 \mathrm{~min} .{ }^{1} \mathbf{H}$ NMR (400 MHz, $\left.\mathbf{C D C l}_{3}\right) \delta$ 7.81-7.76 (m, 4H), 7.43-7.34 (m, 5H), $4.84\left(\mathrm{dd}, J_{1}=12.7 \mathrm{~Hz}, J_{2}=4.0 \mathrm{~Hz}\right.$, $1 \mathrm{H}), 3.05\left(\mathrm{dd}, J_{1}=14.3 \mathrm{~Hz}, J_{2}=4.1 \mathrm{~Hz}, 1 \mathrm{H}\right), 2.95\left(\mathrm{dd}, J_{1}=14.1 \mathrm{~Hz}, J_{2}=12.7 \mathrm{~Hz}, 1 \mathrm{H}\right) .{ }^{13} \mathbf{C ~ N M R}$ (100 MHz, $\left.\mathbf{C D C l}_{3}\right) \delta 179.03,178.31,138.06,136.75,135.76,129.46,129.17,129.05,127.57$, 101.60, 45.12, 36.85. HRMS (ESI) calculated [M+H] for $\mathrm{C}_{16} \mathrm{H}_{13} \mathrm{INOS}$ : 393.9763, found: 393.9760. FTIR (cm $\left.{ }^{-1}\right)$ 2922, 2852, 1696, 1576, 1518, 1389, 1308, 1213, 1178, 1127, 1004, 963, 936.

\section{(S)-2-(4-Bromophenyl)-6-phenyl-5,6-dihydro-4H-1,3-thiazin-4-one (3y)}

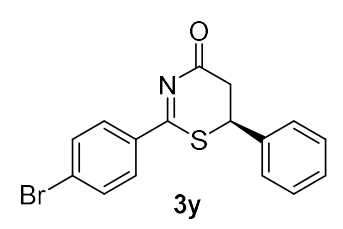

Following the general procedure, treatment of (Z)-2-bromo-3phenylacrylaldehyde $\mathbf{2 a}(63.4 \mathrm{mg}, 0.30 \mathrm{mmol})$ and 4-bromobenzothioamide 1y $(54.0 \mathrm{mg}, 0.25 \mathrm{mmol})$ with triazolium salt $\mathbf{C}(11.6 \mathrm{mg}, 0.025 \mathrm{mmol})$, lithium acetate dihydrate $(5.1 \mathrm{mg}, 0.05 \mathrm{mmol}), \mathrm{K}_{2} \mathrm{CO}_{3}(52.0 \mathrm{mg}, 0.375$ $\mathrm{mmol})$ and $4 \AA \mathrm{MS}(100 \mathrm{mg})$ in mesitylene $(2.0 \mathrm{~mL})$ and stirring the reaction mixture at $25^{\circ} \mathrm{C}$ for 
$72 \mathrm{~h}$ followed by flash column chromatography (Pet. ether-EtOAc: 80:20) to afford $(S)$-2-(4bromophenyl)-6-phenyl-5,6-dihydro-4H-1,3-thiazin-4-one 3y as a pale yellow solid (60 mg, 69\% yield).

$\boldsymbol{R}_{\boldsymbol{f}}($ Pet. ether $/ \mathrm{EtOAc}=80 / 20): 0.5 ; \mathrm{er}=92: 8,[\alpha]_{\mathrm{D}}{ }^{25}=-92.4\left(\mathrm{c} 0.1, \mathrm{CHCl}_{3}\right)$. HPLC $($ Chiralpak IC, 70:30 Hexane / $i$-PrOH, $0.7 \mathrm{~mL} / \mathrm{min}, 254 \mathrm{~nm}$ ) Minor: $40.0 \mathrm{~min}$, Major: $36.0 \mathrm{~min} .{ }^{1} \mathbf{H}$ NMR (400 MHz, $\left.\mathbf{C D C l}_{3}\right) \delta 7.94(\mathrm{~d}, J=8.3 \mathrm{~Hz}, 2 \mathrm{H}), 7.58(\mathrm{~d}, J=8.3 \mathrm{~Hz}, 2 \mathrm{H}), 7.14-7.36(\mathrm{~m}, 5 \mathrm{H}), 4.84\left(\mathrm{dd}, J_{1}\right.$ $\left.=12.9 \mathrm{~Hz}, J_{2}=4.0 \mathrm{~Hz}, 1 \mathrm{H}\right), 3.05\left(\mathrm{dd}, J_{1}=14.2 \mathrm{~Hz}, J_{2}=4.1 \mathrm{~Hz}, 1 \mathrm{H}\right), 2.96(\mathrm{t}, J=13.2 \mathrm{~Hz}, 1 \mathrm{H})$. ${ }^{13}$ C NMR (100 MHz, CDCl $) \delta$ 179.03, 178.04, 136.77, 135.21, 132.18, 132.05, 129.48, 129.21, 128.85, 127.58, 45.18, 36.87. HRMS (ESI) calculated [M+H] for $\mathrm{C}_{16} \mathrm{H}_{13} \mathrm{~B}_{\mathrm{r}} \mathrm{NOS}: 345.9901$, found: 345.9902. FTIR (cm ( $\left.^{-1}\right)$ 3028, 2959, 2922, 2851, 1712, 1661, 1589, 1478, 1407, 1250, 1186, 1070, $1009,907$.

\section{(S)-2-(3-Methoxyphenyl)-6-phenyl-5,6-dihydro-4H-1,3-thiazin-4-one (3z)}

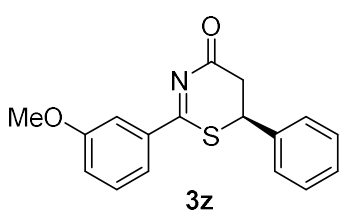

Following the general procedure, treatment of (Z)-2-bromo-3phenylacrylaldehyde $\quad \mathbf{2 a} \quad\left(\begin{array}{llll}63.4 & \mathrm{mg}, & 0.30 & \mathrm{mmol}\end{array}\right)$ and 3 methoxybenzothioamide $\mathbf{1 z}(41.8 \mathrm{mg}, 0.25 \mathrm{mmol})$ with triazolium salt $\mathbf{C}$ (11.6 $\mathrm{mg}, 0.025 \mathrm{mmol})$, lithium acetate dihydrate $(5.1 \mathrm{mg}, 0.05 \mathrm{mmol})$, $\mathrm{K}_{2} \mathrm{CO}_{3}(52.0 \mathrm{mg}, 0.375 \mathrm{mmol})$ and $4 \AA \mathrm{MS}(100 \mathrm{mg})$ in mesitylene $(2.0 \mathrm{~mL})$ and stirring the reaction mixture at $25{ }^{\circ} \mathrm{C}$ for $72 \mathrm{~h}$ followed by flash column chromatography (Pet. ether-EtOAc: 80:20) to afford $(S)$-2-(3-methoxyphenyl)-6-phenyl-5,6-dihydro-4H-1,3-thiazin-4-one $\mathbf{3 z}$ as a yellow oil ( $53 \mathrm{mg}, 72 \%$ yield).

$\boldsymbol{R}_{\boldsymbol{f}}($ Pet. ether $/ \mathrm{EtOAc}=60 / 40): 0.57$; er $=92: 8,[\alpha]_{\mathrm{D}}{ }^{20}=-60.0\left(\mathrm{c} 0.05, \mathrm{CHCl}_{3}\right)$. HPLC $($ Chiralpak IF, 70:30 Hexane / $i$-PrOH, 0.7 mL/min, $254 \mathrm{~nm}$ ) Minor: 26.1 min, Major: 27.4 min. ${ }^{1} \mathbf{H}$ NMR (400 MHz, CDCl $) \delta$ 7.66-7.62 (m, 2H), 7.43-7.32 (m, 6H), 7.14-7.11 (m, 1H), $4.82\left(\mathrm{dd}, J_{1}=12.6\right.$ $\left.\mathrm{Hz}, J_{2}=4.1 \mathrm{~Hz}, 1 \mathrm{H}\right), 3.85(\mathrm{~s}, 3 \mathrm{H}), 3.06\left(\mathrm{dd}, J_{1}=14.2 \mathrm{~Hz}, J_{2}=5.0 \mathrm{~Hz}, 1 \mathrm{H}\right), 2.98\left(\mathrm{dd}, J_{1}=14.0 \mathrm{~Hz}\right.$, $\left.J_{2}=12.6 \mathrm{~Hz}, 1 \mathrm{H}\right) .{ }^{13} \mathbf{C}$ NMR (100 MHz, $\left.\mathbf{C D C l}_{3}\right) \delta 179.32,179.20,159.93,137.78,137.00,129.77$, 129.46, 129.11, 127.61, 120.58, 111.75, 55.64, 45.10, 37.03. HRMS (ESI) calculated [M+H] for $\mathrm{C}_{17} \mathrm{H}_{16} \mathrm{NO}_{2} \mathrm{~S}: 298.0902$, found: 298.0902. FTIR (cm-1) 2932,2836, 1711, 1673, 1597, 1486, 1378, 1286, 1218, 1152, 1041. 
(S)-6-Phenyl-2-(m-tolyl)-5,6-dihydro-4H-1,3-thiazin-4-one (3aa)

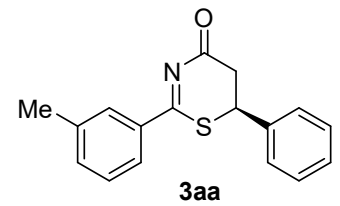

Following the general procedure, treatment of (Z)-2-bromo-3phenylacrylaldehyde $\mathbf{2 a}(63.4 \mathrm{mg}, 0.30 \mathrm{mmol})$ and 3-methylbenzothioamide 1aa $(37.8 \mathrm{mg}, 0.25 \mathrm{mmol})$ with triazolium salt $\mathbf{C}(11.6 \mathrm{mg}, 0.025 \mathrm{mmol})$, lithium acetate dihydrate $(5.1 \mathrm{mg}, 0.05 \mathrm{mmol}), \mathrm{K}_{2} \mathrm{CO}_{3}(52.0 \mathrm{mg}, 0.375$ mmol) and $4 \AA$ MS $(100 \mathrm{mg})$ in mesitylene $(2.0 \mathrm{~mL})$ and stirring the reaction mixture at $25{ }^{\circ} \mathrm{C}$ for $72 \mathrm{~h}$ followed by flash column chromatography (Pet. ether-EtOAc: 80:20) to afford (S)-6-phenyl2-(m-tolyl)-5,6-dihydro-4H-1,3-thiazin-4-one 3aa as a yellow oil (36 mg, 51\% yield).

$\boldsymbol{R}_{\boldsymbol{f}}($ Pet. ether $/ \mathrm{EtOAc}=80 / 20): 0.51 ;$ er $=92: 8,[\alpha]_{\mathrm{D}}{ }^{25}=+62.6\left(\mathrm{c} 0.1, \mathrm{CHCl}_{3}\right)$. HPLC $($ Chiralpak IC, 70:30 Hexane / $i$-PrOH, $0.7 \mathrm{~mL} / \mathrm{min}, 254 \mathrm{~nm}$ ) Minor: $43.3 \mathrm{~min}$, Major: $39.3 \mathrm{~min} .{ }^{1} \mathbf{H}$ NMR $\left(400 \mathrm{MHz}, \mathbf{C D C l}_{3}\right) \delta$ 7.93-7.86 (m, 2H), 7.43-7.32 (m, 7H), $4.82\left(\mathrm{dd}, J_{1}=12.6 \mathrm{~Hz}, J_{2}=4.1 \mathrm{~Hz}\right.$, $1 \mathrm{H}), 3.06\left(\mathrm{dd}, J_{1}=14.3 \mathrm{~Hz}, J_{2}=4.0 \mathrm{~Hz}, 1 \mathrm{H}\right), 2.97\left(\mathrm{dd}, J_{1}=14.0 \mathrm{~Hz}, J_{2}=12.9 \mathrm{~Hz}, 1 \mathrm{H}\right), 2.40(\mathrm{~s}$, 3H). ${ }^{13} \mathbf{C}$ NMR (100 MHz, $\left.\mathbf{C D C l}_{3}\right) \delta$ 179.58, 179.33, 138.80, 137.09, 136.39, 134.65, 129.46, 129.10, 128.71, 127.62, 45.08, 37.05, 21.39. HRMS (ESI) calculated $[\mathrm{M}+\mathrm{H}]$ for $\mathrm{C}_{17} \mathrm{H}_{17} \mathrm{NOS}$ : 282.0953, found: 282.0951. FTIR (cm-1) 3029, 2959, 2922, 2854, 2363, 2329, 1713, 1659, 1606, $1498,1376,1266,1193,1148$.

\section{(S)-2-(3-Bromophenyl)-6-phenyl-5,6-dihydro-4H-1,3-thiazin-4-one (3ab)}

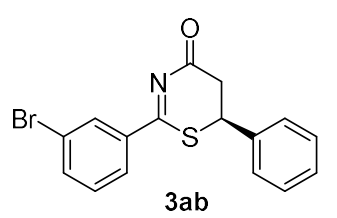

Following the general procedure, treatment of (Z)-2-bromo-3phenylacrylaldehyde $\mathbf{2 a b} \quad\left(\begin{array}{llll}63.4 & \mathrm{mg}, & 0.30 & \mathrm{mmol}\end{array}\right)$ and 3bromobenzothioamide C (54.0 mg, $0.25 \mathrm{mmol})$ with triazolium salt $\mathbf{C}$ (11.6 $\mathrm{mg}, 0.025 \mathrm{mmol})$, lithium acetate dihydrate $(5.1 \mathrm{mg}, 0.05 \mathrm{mmol}), \mathrm{K}_{2} \mathrm{CO}_{3}$ $(52.0 \mathrm{mg}, 0.375 \mathrm{mmol})$ and $4 \AA \mathrm{MS}(100 \mathrm{mg})$ in mesitylene $(2.0 \mathrm{~mL})$ and stirring the reaction mixture at $25^{\circ} \mathrm{C}$ for $72 \mathrm{~h}$ followed by flash column chromatography (Pet. ether-EtOAc: 80:20) to afford (S)-2-(3-bromophenyl)-6-phenyl-5,6-dihydro-4H-1,3-thiazin-4-one 3ab as a pale yellow solid (70 mg, 81\% yield).

$\boldsymbol{R}_{\boldsymbol{f}}($ Pet. ether $/$ EtOAc $=70 / 30): 0.58$; er $=92: 8,[\alpha]_{\mathrm{D}}{ }^{25}=-60.8\left(\mathrm{c} 0.1, \mathrm{CHCl}_{3}\right)$. HPLC $($ Chiralpak IC, 70:30 Hexane / $i$-PrOH, $0.7 \mathrm{~mL} / \mathrm{min}, 254 \mathrm{~nm}$ ) Minor: $39.3 \mathrm{~min}$, Major: $35.8 \mathrm{~min} .{ }^{1} \mathbf{H}$ NMR (400 MHz, $\left.\mathbf{C D C l}_{3}\right) \delta 8.24(\mathrm{t}, J=1.4 \mathrm{~Hz}, 1 \mathrm{H}), 7.98-7.96(\mathrm{~m}, 1 \mathrm{H}), 7.70-7.67(\mathrm{~m}, 1 \mathrm{H}), 7.29-7.43(\mathrm{~m}$, $6 \mathrm{H}), 4.85\left(\mathrm{dd}, J_{1}=12.7 \mathrm{~Hz}, J_{2}=4.2 \mathrm{~Hz}, 1 \mathrm{H}\right), 3.06\left(\mathrm{dd}, J_{1}=14.2 \mathrm{~Hz}, J_{2}=4.1 \mathrm{~Hz}, 1 \mathrm{H}\right), 2.97(\mathrm{dd}$, $\left.J_{1}=14.0 \mathrm{~Hz}, J_{2}=12.7 \mathrm{~Hz}, 1 \mathrm{H}\right) .{ }^{13} \mathbf{C} \mathbf{N M R}\left(\mathbf{1 0 0} \mathbf{M H z}, \mathbf{C D C l}_{3}\right) \delta 178.93,177.68,138.18,136.66$, 
136.36, 130.60, 130.28, 129.51, 129.22, 127.58, 126.29, 123.07, 45.25, 36.82. HRMS (ESI) calculated $[\mathrm{M}+\mathrm{H}]$ for $\mathrm{C}_{16} \mathrm{H}_{13} \mathrm{BrNOS}$ : 345.9901, found: 345.9904. FTIR (cm $\left.{ }^{-1}\right)$ 3028, 2922, 1713, $1658,1562,1498,1380,1243,1149,1074,748,697$.

\section{(S)-2-(3-Nitrophenyl)-6-phenyl-5,6-dihydro-4H-1,3-thiazin-4-one (3ac)}

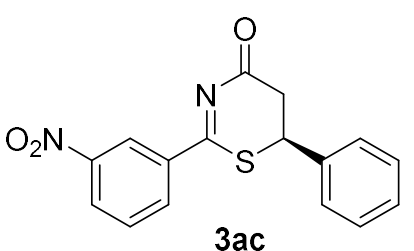

Following the general procedure, treatment of (Z)-2-bromo-3phenylacrylaldehyde $\mathbf{2 a}\left(\begin{array}{llll}63.4 & \mathrm{mg}, & 0.30 \mathrm{mmol}) & \text { and } 3-\end{array}\right.$ nitrobenzothioamide 1ac $(45.5 \mathrm{mg}, 0.25 \mathrm{mmol})$ with triazolium salt C (11.6 mg, $0.025 \mathrm{mmol})$, lithium acetate dihydrate $(5.1 \mathrm{mg}, 0.05$

$\mathrm{mmol}), \mathrm{K}_{2} \mathrm{CO}_{3}(52.0 \mathrm{mg}, 0.375 \mathrm{mmol})$ and $4 \AA \mathrm{MS}(100 \mathrm{mg})$ in mesitylene $(2.0 \mathrm{~mL})$ and stirring the reaction mixture at $25{ }^{\circ} \mathrm{C}$ for $72 \mathrm{~h}$ followed by flash column chromatography (Pet. etherEtOAc: $85: 15)$ to afford(S)-2-(3-nitrophenyl)-6-phenyl-5,6-dihydro-4H-1,3-thiazin-4-one 3ac as a pale yellow solid(39 $\mathrm{mg}, 50 \%$ yield).

$\boldsymbol{R}_{f}($ Pet. ether $/ \mathrm{EtOAc}=70 / 30): 0.53 ; \mathrm{er}=92: 8,[\alpha]_{\mathrm{D}}{ }^{20}=-62.0\left(\mathrm{c} 0.05, \mathrm{CHCl}_{3}\right)$. HPLC $($ ChiralpakIF, 70:30 Hexane / $i$-PrOH, $0.7 \mathrm{~mL} / \mathrm{min}, 254 \mathrm{~nm}$ ) Minor: 50.7 min, Major: 57.4 min. ${ }^{1}$ H NMR (400 MHz, $\left.\mathbf{C D C l}_{3}\right) \delta 8.32-8.23(\mathrm{~m}, 4 \mathrm{H}), 7.44-7.38(\mathrm{~m}, 5 \mathrm{H}), 4.92\left(\mathrm{dd}, J_{1}=12.6 \mathrm{~Hz}, J_{2}=4.1 \mathrm{~Hz}, 1 \mathrm{H}\right)$,

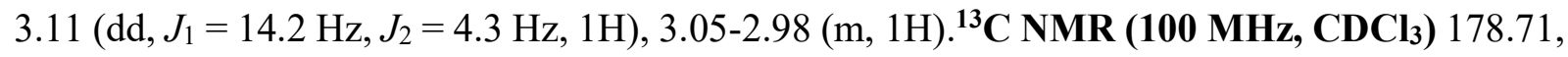
$176.81,150.76,141.71,136.34,129.67,129.48,128.85,127.62,124.00,45.74,36.67$. HRMS (ESI) calculated [M+H] for $\mathrm{C}_{16} \mathrm{H}_{13} \mathrm{~N}_{2} \mathrm{O}_{3} \mathrm{~S}: 313.0647$, found: 313.0647. FTIR (cm $\left.{ }^{-1}\right)$ 2961, 2923, 2854, 1716, 1603, 1525, 1383, 1347, 1249, 1186, 1107, 916.

\section{(S)-2-(2-Fluorophenyl)-6-phenyl-5,6-dihydro-4H-1,3-thiazin-4-one (3ad)}

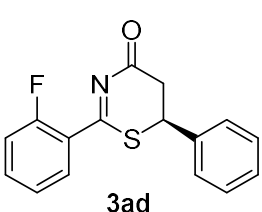

Following the general procedure, treatment of (Z)-2-bromo-3phenylacrylaldehyde $\mathbf{2 a}(63.4 \mathrm{mg}, 0.30 \mathrm{mmol})$ and 2-fluorobenzothioamide 1ad (38.8 $\mathrm{mg}, 0.25 \mathrm{mmol})$ with triazolium salt $\mathbf{C}(11.6 \mathrm{mg}, 0.025 \mathrm{mmol})$, lithium acetate dihydrate $(5.1 \mathrm{mg}, 0.05 \mathrm{mmol}), \mathrm{K}_{2} \mathrm{CO}_{3}(52.0 \mathrm{mg}, 0.375 \mathrm{mmol})$ and $4 \AA$ MS $(100 \mathrm{mg})$ in mesitylene $(2.0 \mathrm{~mL})$ and stirring the reaction mixture at $25^{\circ} \mathrm{C}$ for $72 \mathrm{~h}$ followed by flash column chromatography (Pet. ether-EtOAc: 85:15) to afford (S)-2-(2-fluorophenyl)-6phenyl-5,6-dihydro-4H-1,3-thiazin-4-one (3ad) 3ad as a pale yellow solid (46 mg, 65\% yield). $\boldsymbol{R}_{\boldsymbol{f}}($ Pet. ether $/ \mathrm{EtOAc}=80 / 20): 0.52 ; \mathrm{er}=90: 10,[\alpha]_{\mathrm{D}}{ }^{25}=+136.9\left(\mathrm{c} 0.1, \mathrm{CHCl}_{3}\right)$. HPLC (Chiralpak IA, 80:20 Hexane / $i$-PrOH, $0.7 \mathrm{~mL} / \mathrm{min}, 254 \mathrm{~nm}$ ) Minor: $15.9 \mathrm{~min}$, Major: $14.2 \mathrm{~min} .{ }^{1} \mathbf{H}$ NMR 


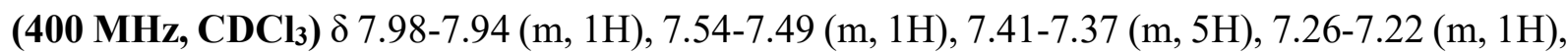
7.18-7.14 (m, 1H), $4.86\left(\mathrm{dd}, J_{1}=12.2 \mathrm{~Hz}, J_{2}=4.6 \mathrm{~Hz}, 1 \mathrm{H}\right), 3.10-2.98(\mathrm{~m}, 2 \mathrm{H}) .{ }^{13} \mathbf{C}$ NMR (100 MHz, $\left.\mathbf{C D C l}_{3}\right) \delta 178.48,176.17(\mathrm{~d}, J=4.3 \mathrm{~Hz}), 160.8(\mathrm{~d}, J=257 \mathrm{~Hz}), 136.72,134.21(\mathrm{~d}, J=9.2$ $\mathrm{Hz}), 130.68,130.33,129.49,129.16,127.65,124.90,124.55,124.52,116.96$ (d, $J=22.2 \mathrm{~Hz})$, 109.37, 77.48, 77.16, 76.84, $45.82(\mathrm{~d}, J=3.5 \mathrm{~Hz})$. HRMS (ESI) calculated [M+H] for $\mathrm{C}_{16} \mathrm{H}_{13}$ FNOS: 286.0702, found: 286.0702. FTIR (cm $\left.{ }^{-1}\right)$ 2922, 2852, 1645, 1521, 1485, 1451, $1373,1277,1225,1153,1022$.

\section{(S)-2-(Naphthalen-1-yl)-6-phenyl-5,6-dihydro-4H-1,3-thiazin-4-one (3ae)}

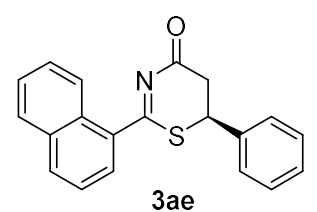

Following the general procedure, treatment of (Z)-2-bromo-3phenylacrylaldehyde $\mathbf{1 a}(63.4 \mathrm{mg}, \quad 0.30 \mathrm{mmol})$ and naphthalene-1carbothioamide 2 ae $(46.8 \mathrm{mg}, 0.25 \mathrm{mmol})$ with triazolium salt C (11.6 mg, $0.025 \mathrm{mmol})$, lithium acetate dihydrate $(5.1 \mathrm{mg}, 0.05 \mathrm{mmol}), \mathrm{K}_{2} \mathrm{CO}_{3}(52.0 \mathrm{mg}$, $0.375 \mathrm{mmol})$ and $4 \AA \mathrm{MS}(100 \mathrm{mg})$ in mesitylene $(2.0 \mathrm{~mL})$ and stirring the reaction mixture at 25 ${ }^{\circ} \mathrm{C}$ for $72 \mathrm{~h}$ followed by flash column chromatography (Pet. ether-EtOAc: 80:20) to afford $(S)$-2(naphthalen-1-yl)-6-phenyl-5,6-dihydro-4H-1,3-thiazin-4-one 3ae as a pale yellow solid (50 mg, $63 \%$ yield).

$\boldsymbol{R}_{f}($ Pet. ether $/$ EtOAc $=70 / 30): 0.63$; er $=91: 9,[\alpha]_{\mathrm{D}}^{25}=-54.4\left(\right.$ c $\left.0.1, \mathrm{CHCl}_{3}\right)$. HPLC $($ Chiralpak IC, 60:40 Hexane / i-PrOH, 0.7 mL/min, $254 \mathrm{~nm}$ ) Minor: 39.2 min, Major: 34.5 min. ${ }^{1}$ H NMR $\left(400 \mathrm{MHz}, \mathbf{C D C l}_{3}\right) \delta 8.54(\mathrm{~d}, \mathrm{~J}=8.4 \mathrm{~Hz}, 1 \mathrm{H}), 7.99(\mathrm{~d}, J=8.1 \mathrm{~Hz}, 1 \mathrm{H}), 7.89-7.82(\mathrm{~m}, 2 \mathrm{H}), 7.62-$ $7.54(\mathrm{~m}, 2 \mathrm{H}), 7.52-7.47(\mathrm{~m}, 1 \mathrm{H}), 7.42-7.37(\mathrm{~m}, 5 \mathrm{H}), 4.91\left(\mathrm{dd}, J_{1}=11.8 \mathrm{~Hz}, J_{2}=5.1 \mathrm{~Hz}, 1 \mathrm{H}\right), 3.16-$ 3.05 (m, 2H). ${ }^{13} \mathbf{C}$ NMR (100 MHz, $\left.\mathbf{C D C l}_{3}\right) \delta 181.30,178.66,140.32,136.85,134.48,133.90$, $133.60,132.62$, 129.52, 129.09, 128.59, 127.58, 126.82, 125.18, 125.14, 124.66, 124.58, 45.88, 36.62. HRMS (ESI) calculated [M+H] for $\mathrm{C}_{20} \mathrm{H}_{16} \mathrm{NOS}: 318.0953$, found: 318.0958 . FTIR (cm $\left.{ }^{-1}\right)$ 3057, 2922, 2852, 1709, 1649, 1507, 1453, 1413, 1374, 1247, 1194, 1149, 1029.

\section{(S)-6-Phenyl-2-(thiophen-2-yl)-5,6-dihydro-4H-1,3-thiazin-4-one (3af)}

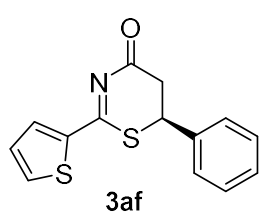

Following the general procedure, treatment of (Z)-2-bromo-3phenylacrylaldehyde $\mathbf{2 a}(63.4 \mathrm{mg}, 0.30 \mathrm{mmol})$ and thiophene-2-carbothioamide 1af $(35.8 \mathrm{mg}, 0.25 \mathrm{mmol})$ with triazolium salt $\mathbf{C}(11.6 \mathrm{mg}, 0.025 \mathrm{mmol})$, lithium acetate dihydrate $(5.1 \mathrm{mg}, 0.05 \mathrm{mmol}), \mathrm{K}_{2} \mathrm{CO}_{3}(52.0 \mathrm{mg}, 0.375 \mathrm{mmol})$ and $4 \AA$ 
MS (100 mg) in mesitylene $(2.0 \mathrm{~mL})$ and stirring the reaction mixture at $25{ }^{\circ} \mathrm{C}$ for $72 \mathrm{~h}$ followed by flash column chromatography (Pet. ether-EtOAc: 80:20) to afford $(S)$-6-phenyl-2-(thiophen-2yl)-5,6-dihydro-4H-1,3-thiazin-4-one 3af as a yellow oil (34 mg, $49 \%$ yield).

$\boldsymbol{R}_{\boldsymbol{f}}($ Pet. ether $/$ EtOAc $=80 / 20): 0.41$; er $=86: 14,[\alpha]_{\mathrm{D}}{ }^{25}=-42.6\left(\mathrm{c} 0.1, \mathrm{CHCl}_{3}\right)$. HPLC $($ Chiralpak IC, 70:30 Hexane / i-PrOH, $0.7 \mathrm{~mL} / \mathrm{min}, 254 \mathrm{~nm}$ ) Minor: $57.5 \mathrm{~min}$, Major: $53.4 \mathrm{~min} .{ }^{1} \mathbf{H}$ NMR (400 MHz, $\left.\mathbf{C D C l}_{3}\right) \delta$ 7.86-7.85 (m, 1H), 7.69-7.67 (m, 1H), 7.43-7.35 (m, 5H), 7.15-7.13 (m, 1H), $4.84\left(\mathrm{dd}, J_{1}=12.5 \mathrm{~Hz}, J_{2}=4.0 \mathrm{~Hz}, 1 \mathrm{H}\right), 3.08\left(\mathrm{dd}, J_{1}=14.5 \mathrm{~Hz}, J_{2}=4.0 \mathrm{~Hz}, 1 \mathrm{H}\right), 2.98\left(\mathrm{dd}, J_{1}=\right.$ $\left.14.7 \mathrm{~Hz}, J_{2}=12.5 \mathrm{~Hz}, 1 \mathrm{H}\right) .{ }^{13} \mathbf{C}$ NMR (100 MHz, $\left.\mathbf{C D C l}_{3}\right) \delta 178.77,172.33,141.51,136.96$, 131.81, 129.47, 129.15, 128.59, 128.54, 127.61, 45.23, 37.67. HRMS (ESI) calculated [M+H] for $\mathrm{C}_{14} \mathrm{H}_{12} \mathrm{NO} \mathrm{S}$ 2: 274.0360, found: 274.0359. FTIR (cm-1) 2960, 2923, 2852, 1711, 1651, 1523, $1489,1419,1260,1149,728,697$. 


\section{7. ${ }^{1} \mathrm{H}$ and ${ }^{13} \mathrm{C}$ NMR Spectra of Functionalized Thiazinones}

(S)-2,6-Diphenyl-5,6-dihydro-4H-1,3-thiazin-4-one (3a)
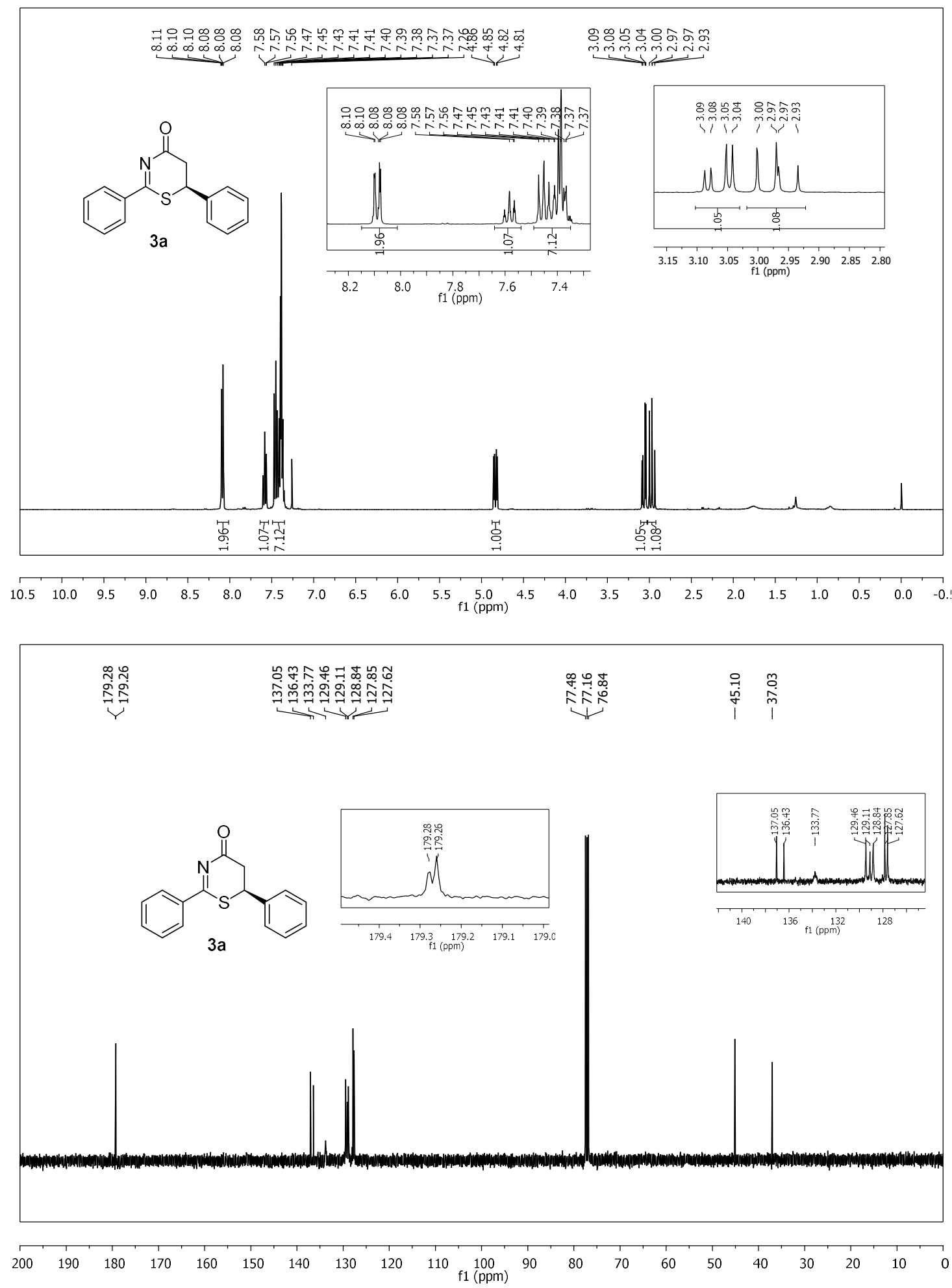
(S)-6-(4-Methoxyphenyl)-2-phenyl-5,6-dihydro-4H-1,3-thiazin-4-one (3b)
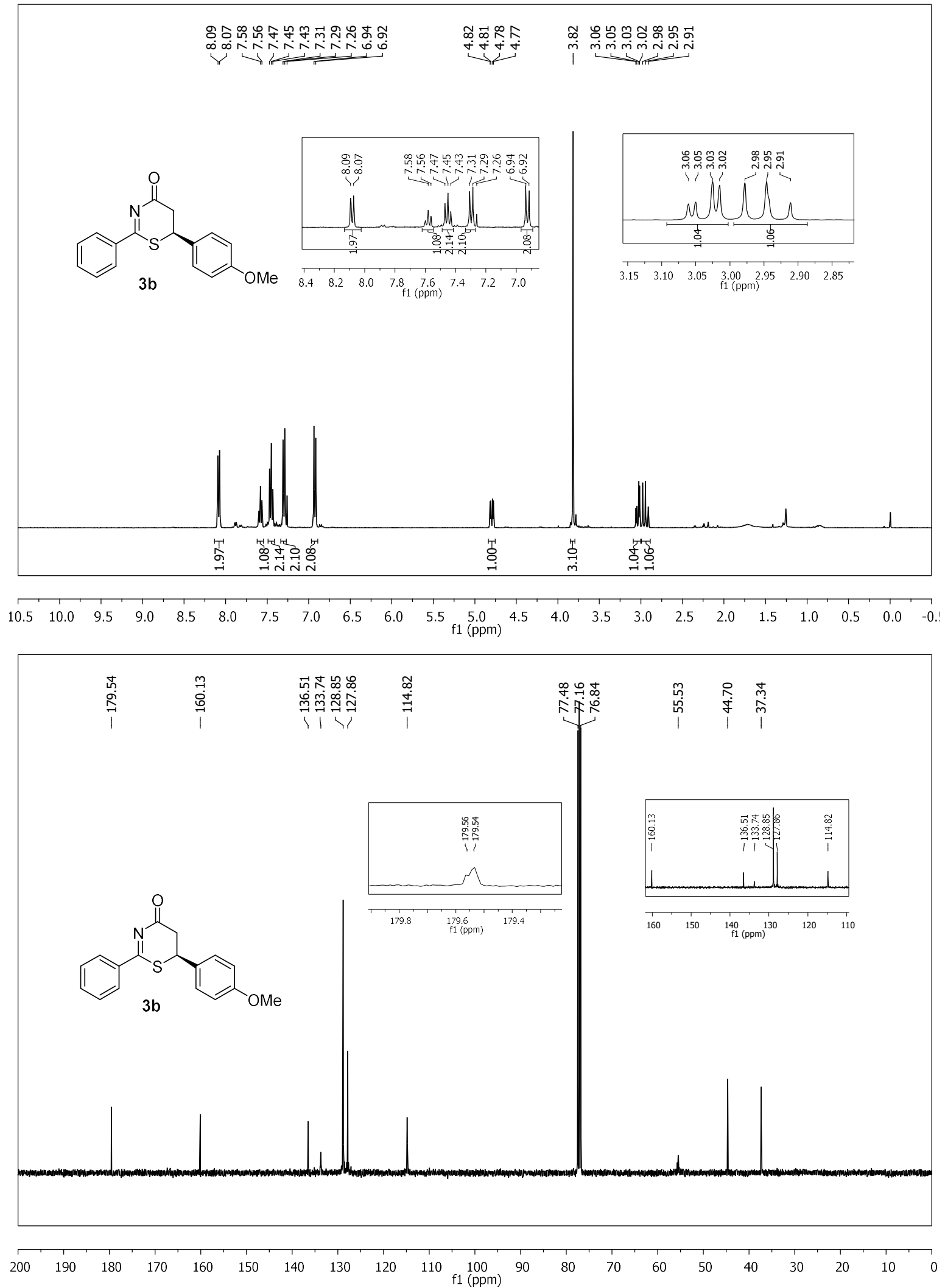
(S)-2-Phenyl-6-(p-tolyl)-5,6-dihydro-4H-1,3-thiazin-4-one (3c)
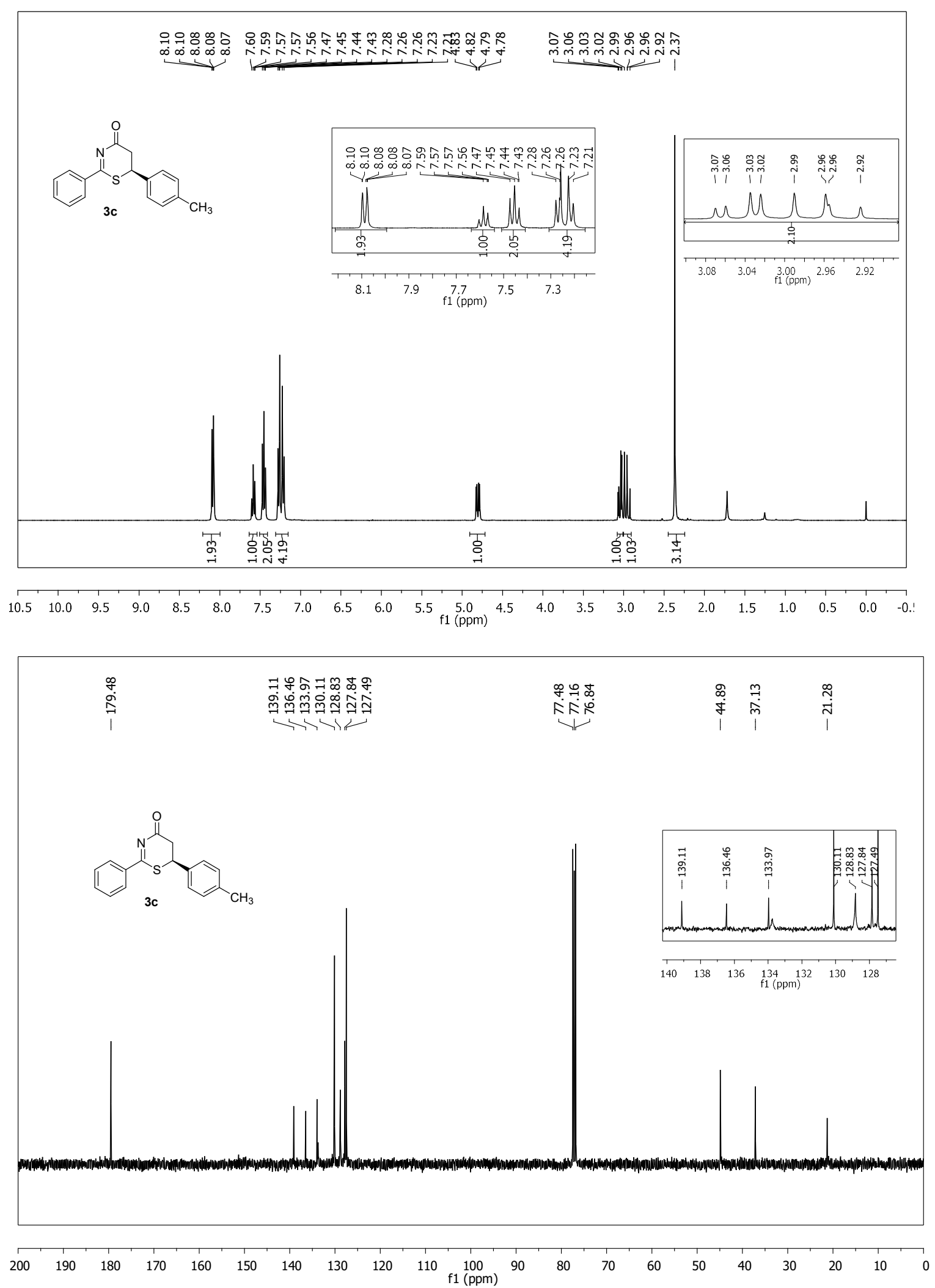
(S)-6-(4-Bromophenyl)-2-phenyl-5,6-dihydro-4H-1,3-thiazin-4-one (3d)
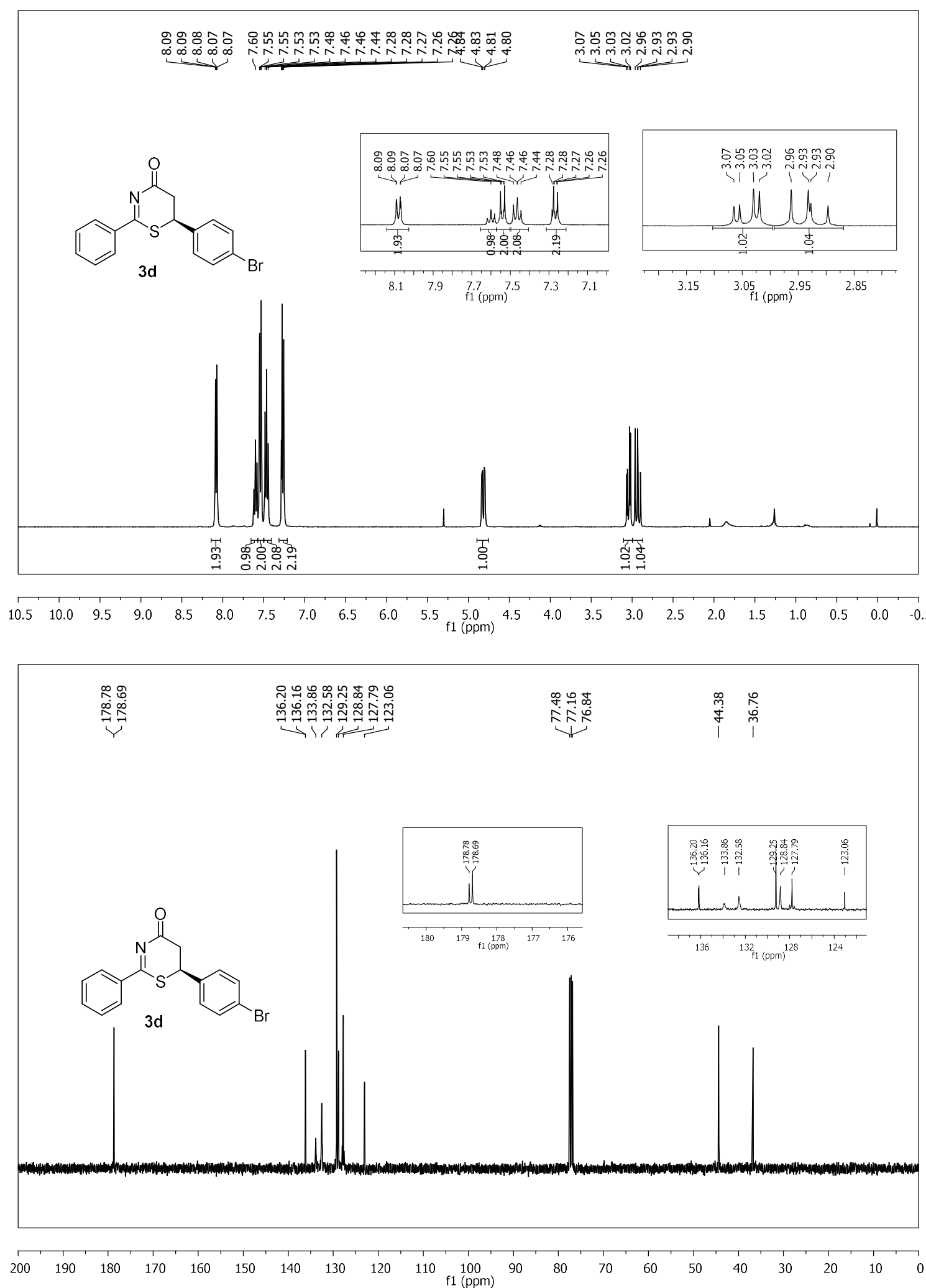
(S)-6-(4-Chlorophenyl)-2-phenyl-5,6-dihydro-4H-1,3-thiazin-4-one (3e)
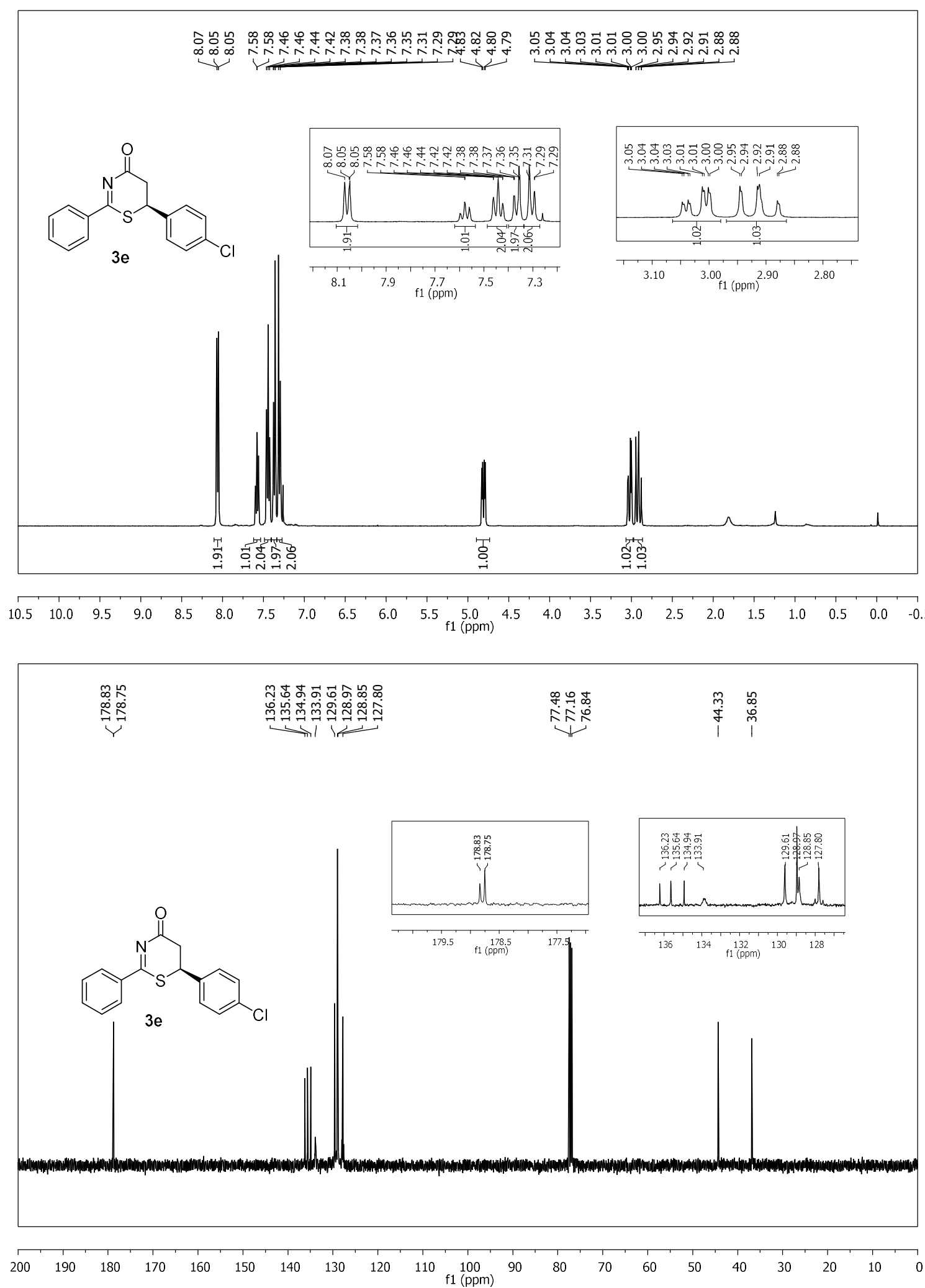
(S)-6-(4-Fluorophenyl)-2-phenyl-5,6-dihydro-4H-1,3-thiazin-4-one (3f)
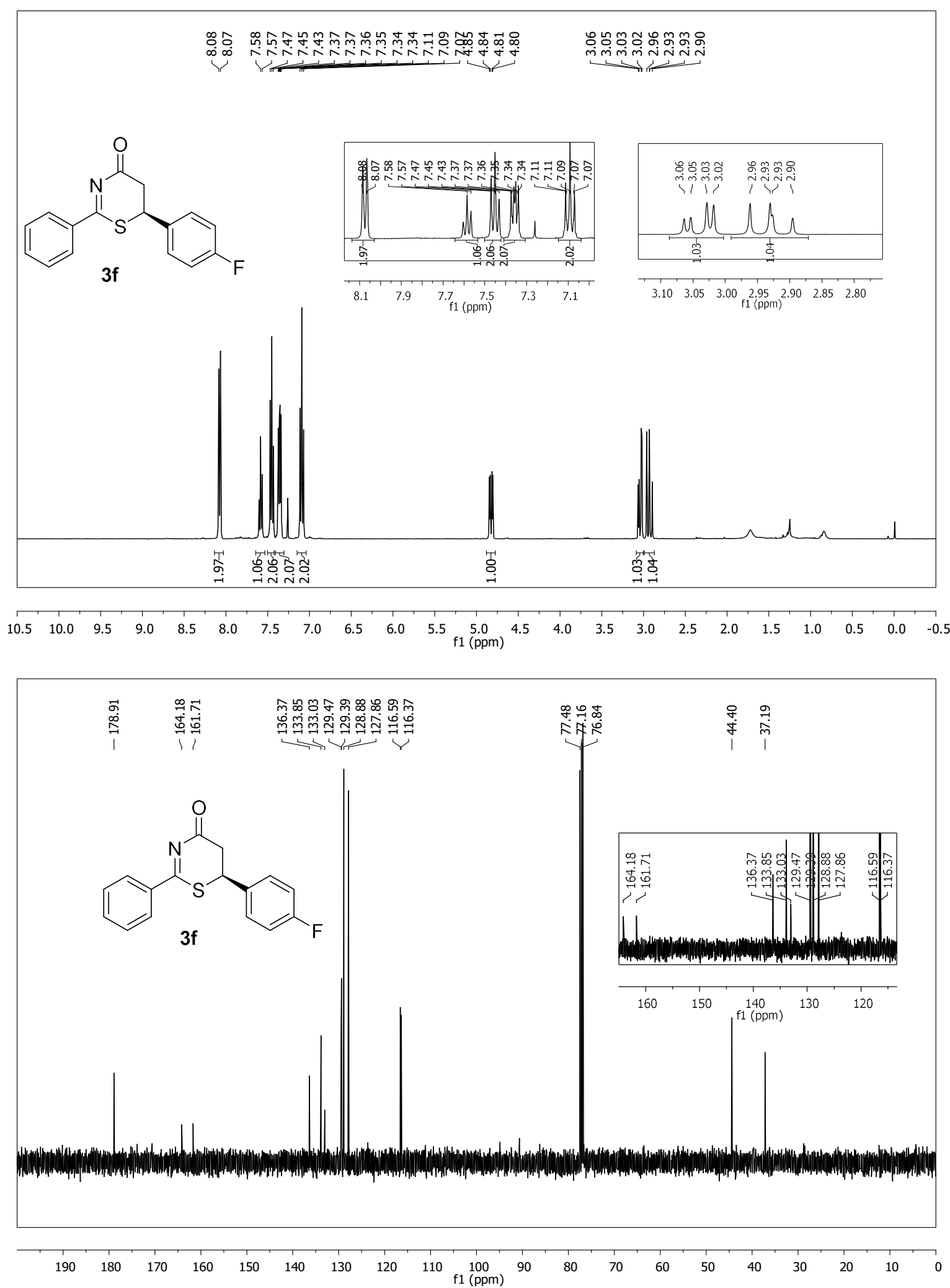
(S)-2-Phenyl-6-(4-(trifluoromethyl)phenyl)-5,6-dihydro-4H-1,3-thiazin-4-one (3g)

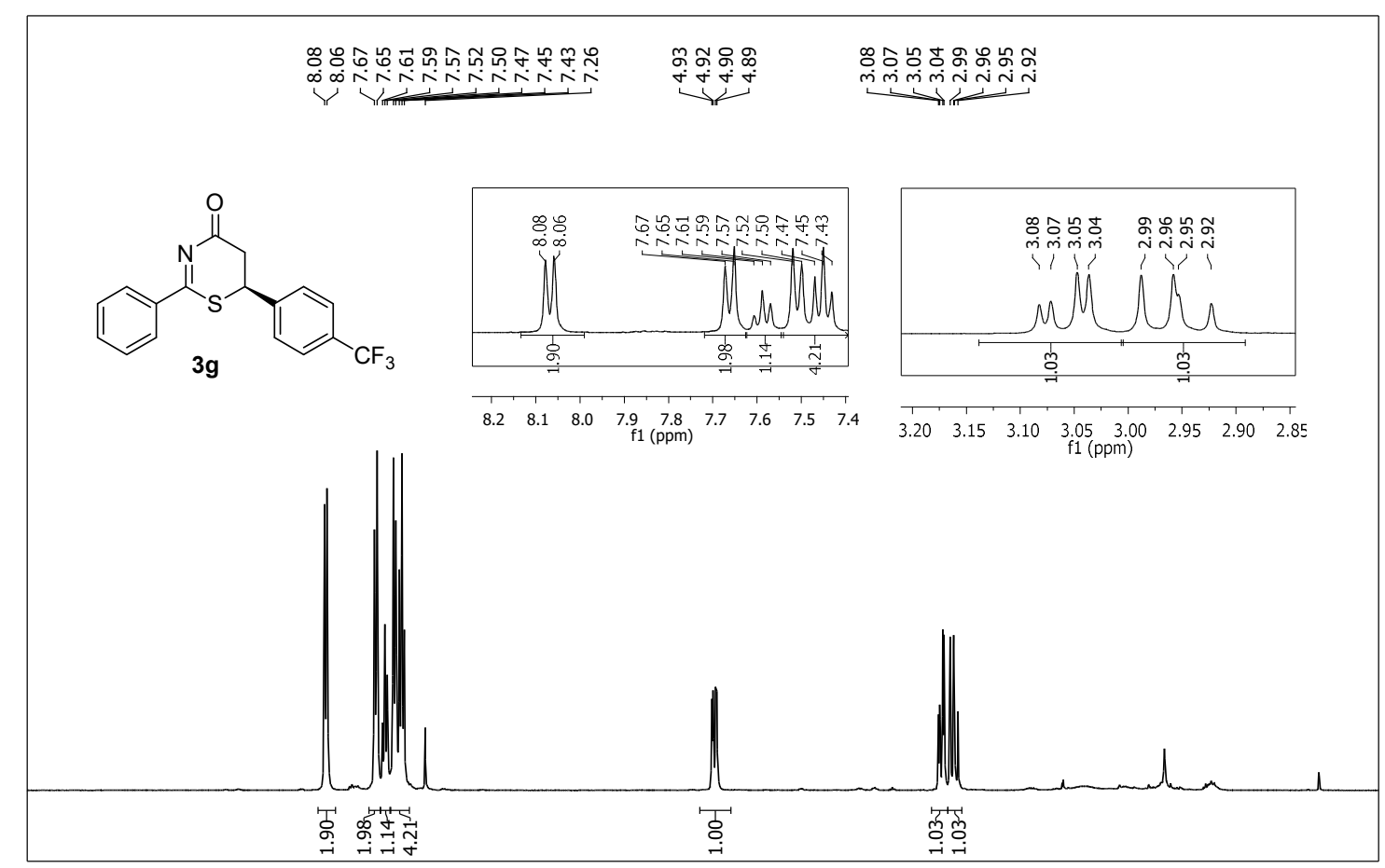

\begin{tabular}{rllllllllllllllllllllllll}
\hline 10.5 & 10.0 & 9.5 & 9.0 & 8.5 & 8.0 & 7.5 & 7.0 & 6.5 & 6.0 & 5.5 & $\begin{array}{c}5.0 \\
\mathrm{f} 1\end{array}(\mathrm{ppm})$ & 4.5 & 4.0 & 3.5 & 3.0 & 2.5 & 2.0 & 1.5 & 1.0 & 0.5 & 0.0 & -0.
\end{tabular}

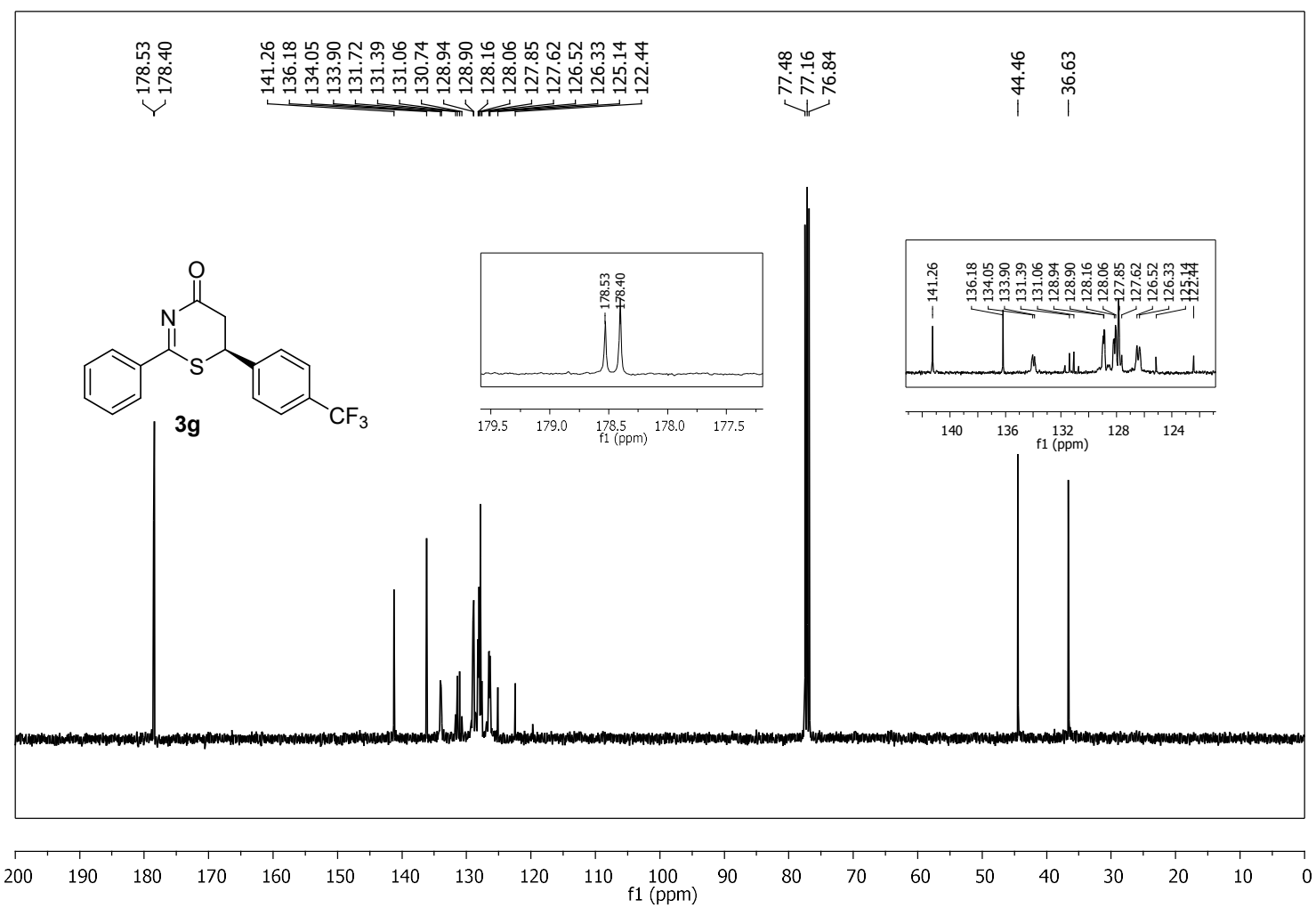


(S)-6-(4-Nitrophenyl)-2-phenyl-5,6-dihydro-4H-1,3-thiazin-4-one (3h)
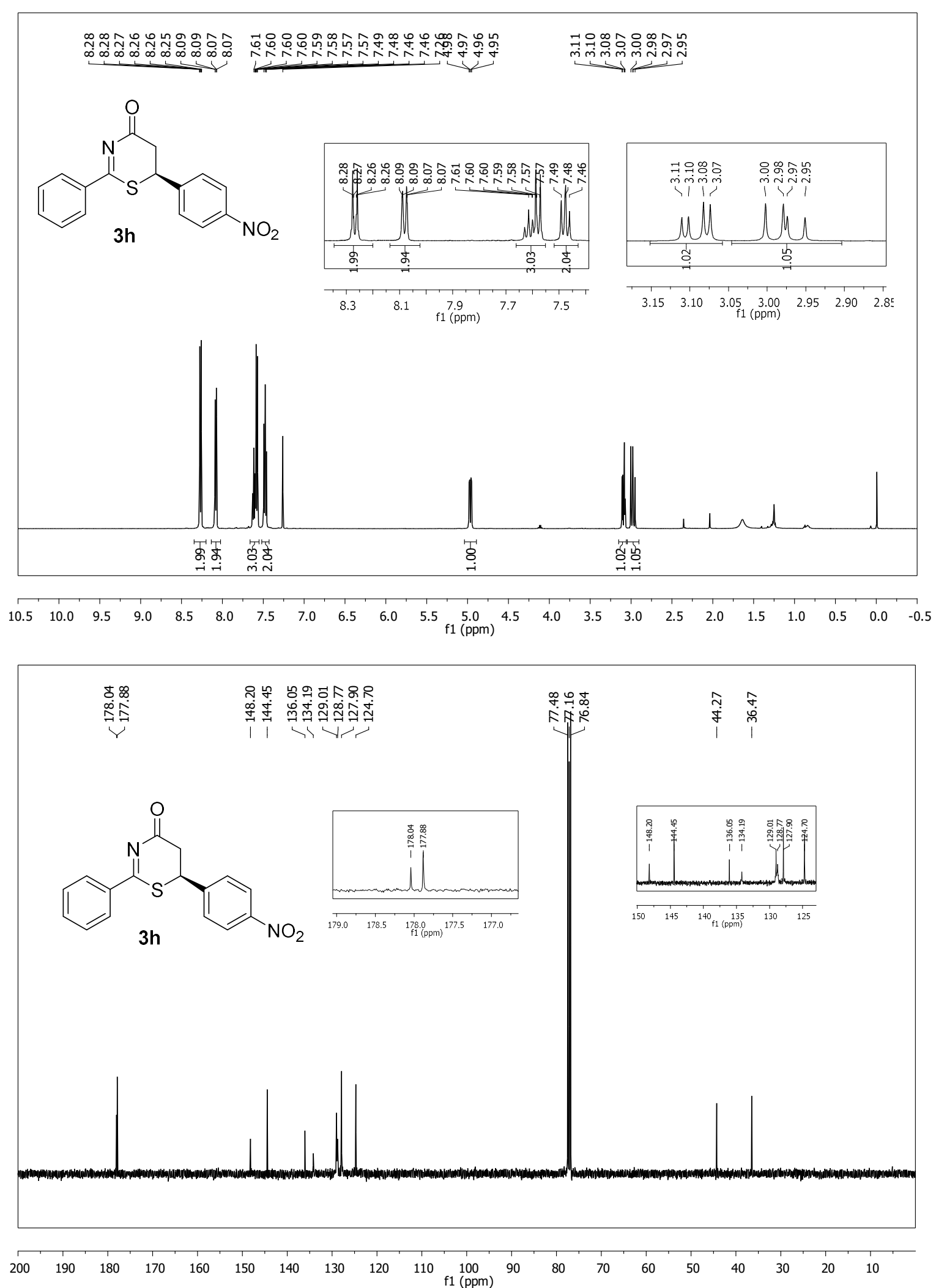
(S)-6-(3-Bromophenyl)-2-phenyl-5,6-dihydro-4H-1,3-thiazin-4-one (3i)
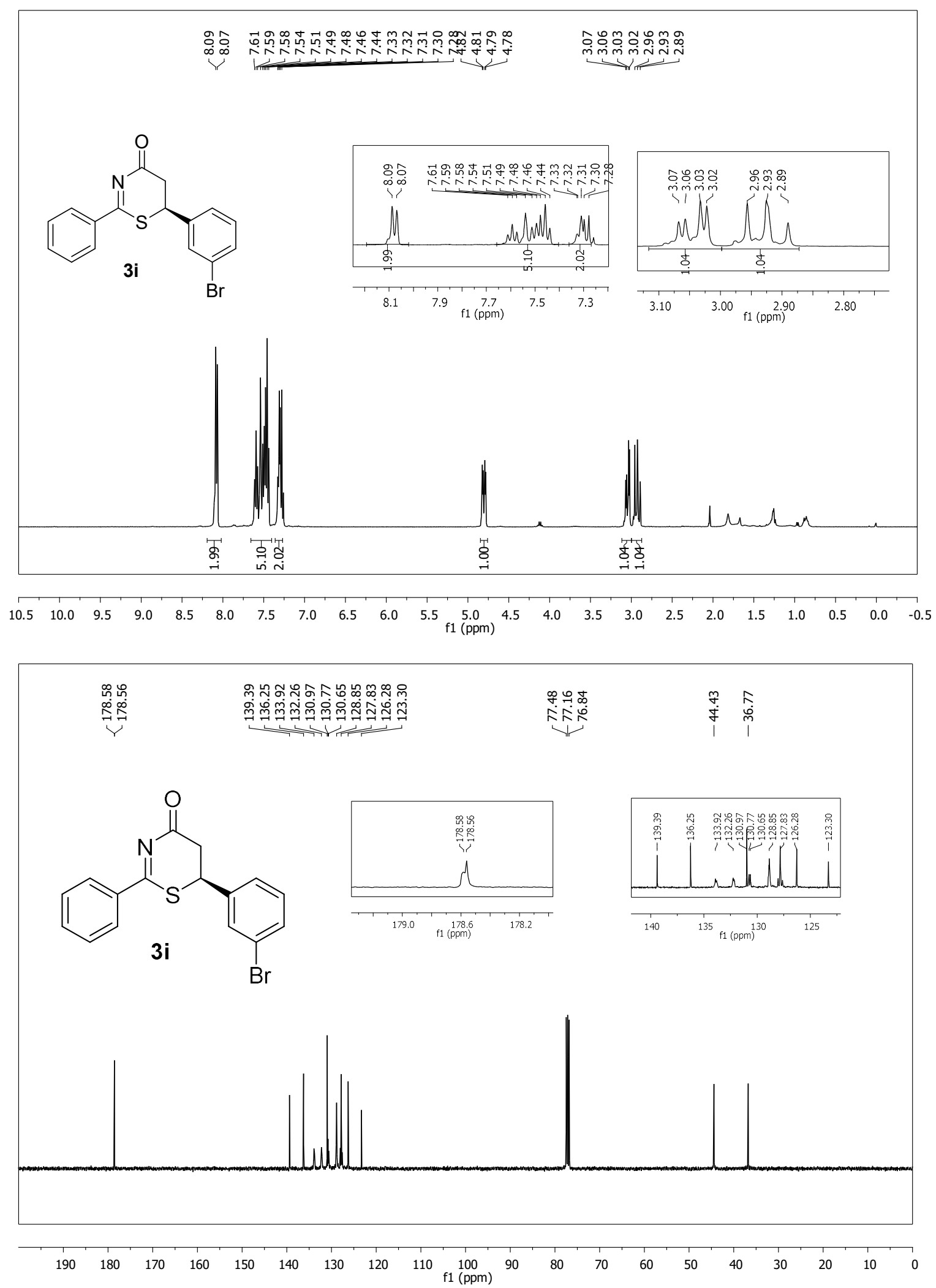
(S)-6-(3-Chlorophenyl)-2-phenyl-5,6-dihydro-4H-1,3-thiazin-4-one (3j)

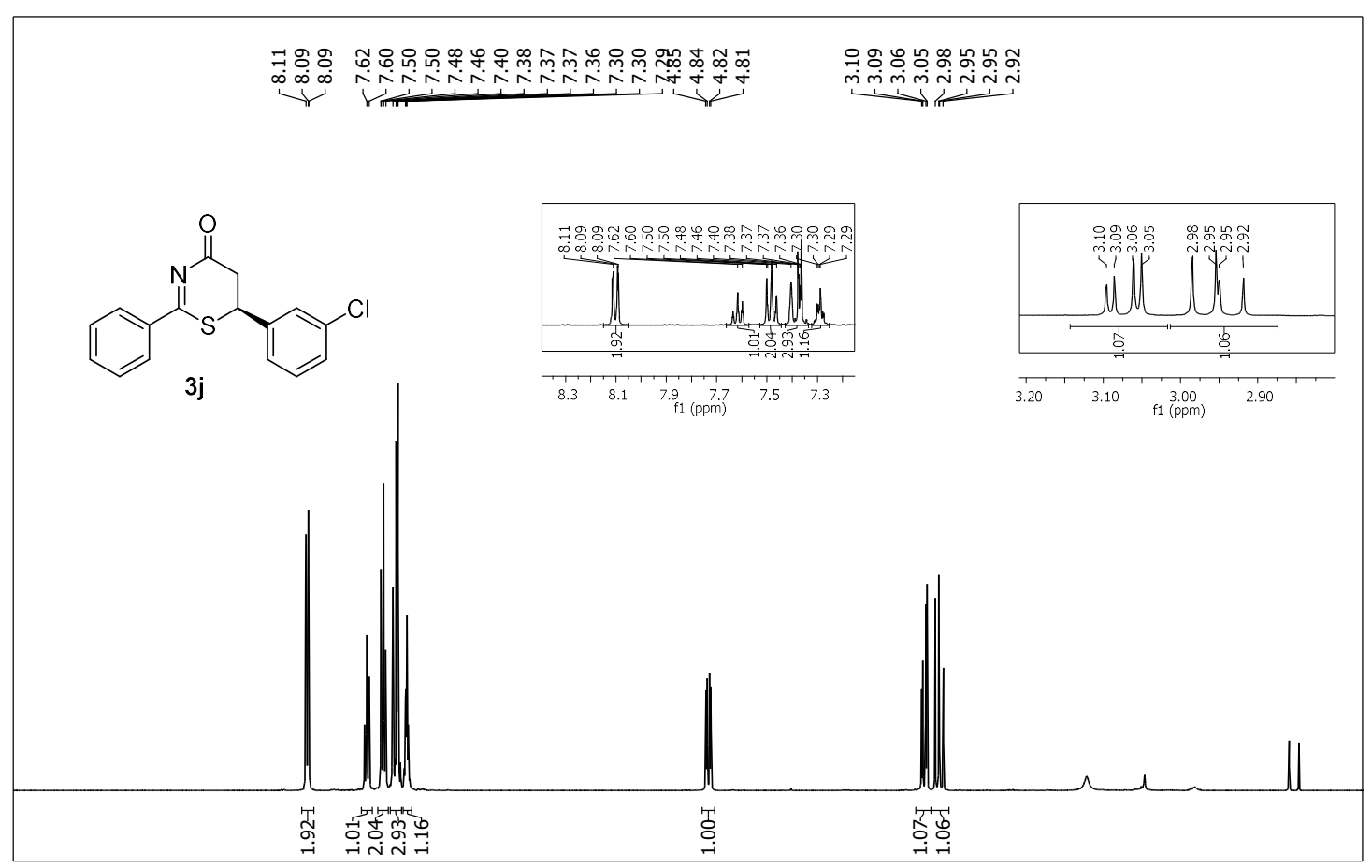

$\begin{array}{rllllllllllllllllllllll}10.5 & 10.0 & 9.5 & 9.0 & 8.5 & 8.0 & 7.5 & 7.0 & 6.5 & 6.0 & 5.5 & \underset{f 1}{5(\mathrm{ppm})} & 4.5 & 4.0 & 3.5 & 3.0 & 2.5 & 2.0 & 1.5 & 1.0 & 0.5 & 0.0\end{array}$

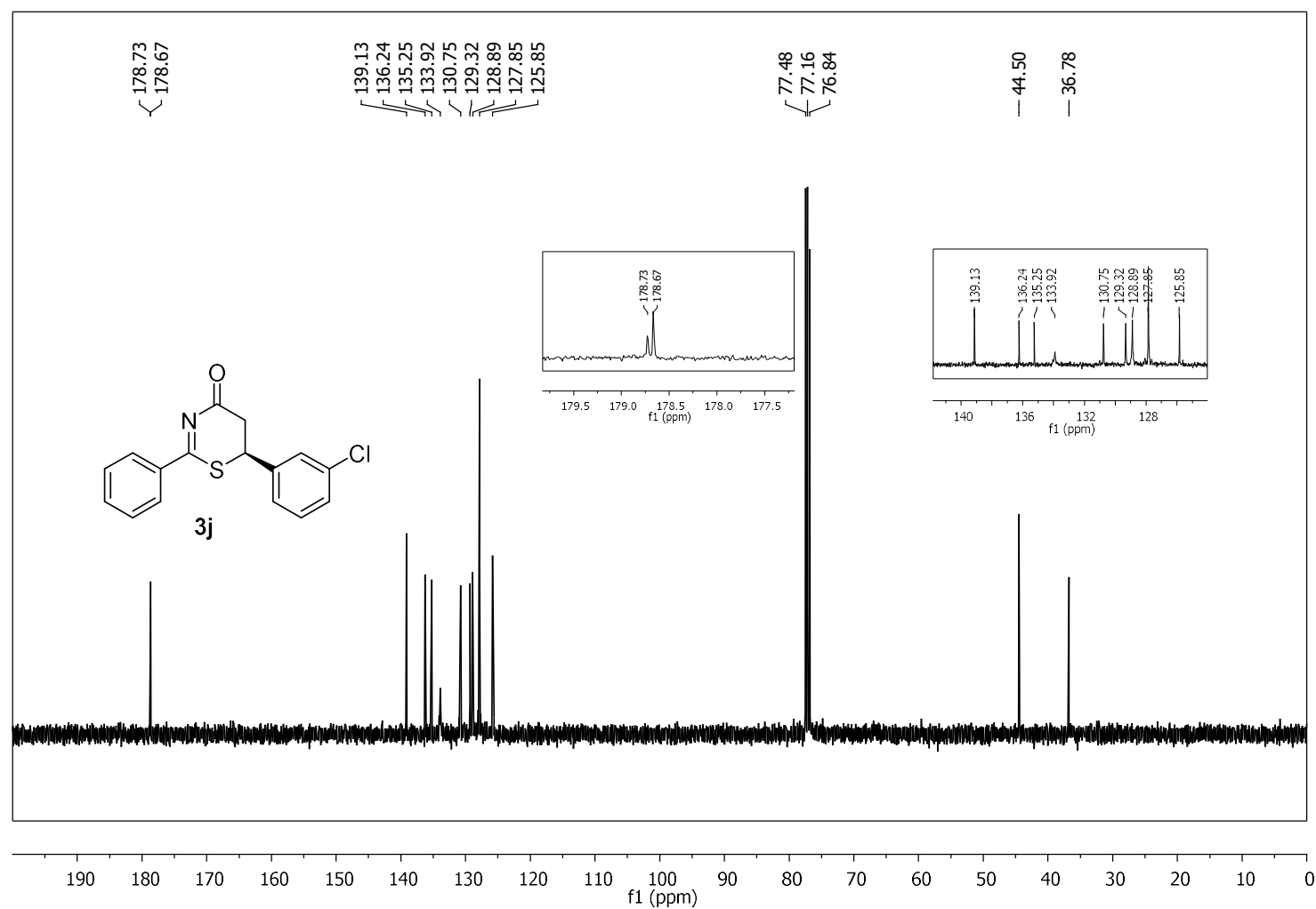


(S)-6-(3-Nitrophenyl)-2-phenyl-5,6-dihydro-4H-1,3-thiazin-4-one (3k)
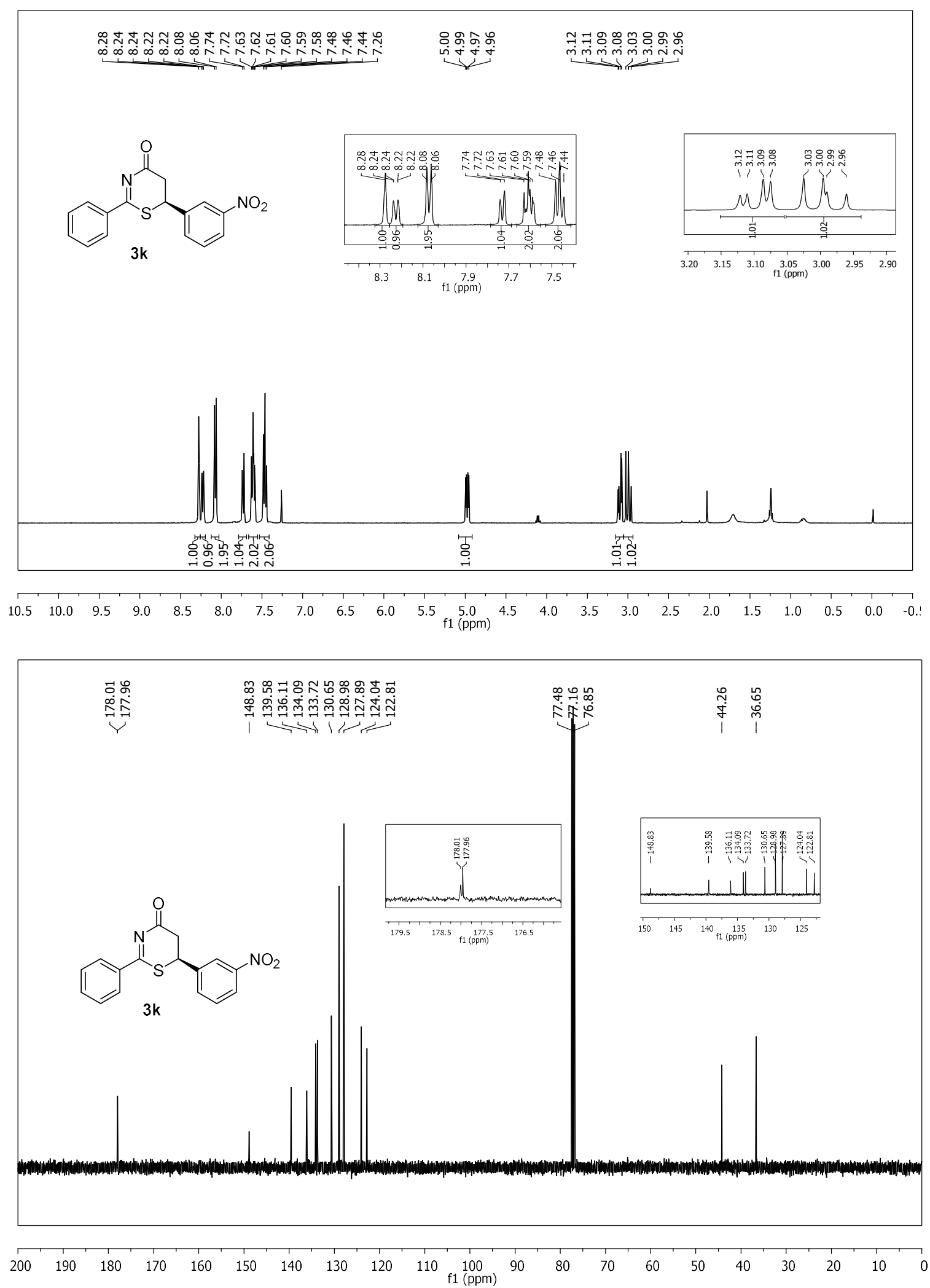
(S)-6-(2-Methoxyphenyl)-2-phenyl-5,6-dihydro-4H-1,3-thiazin-4-one (3I)
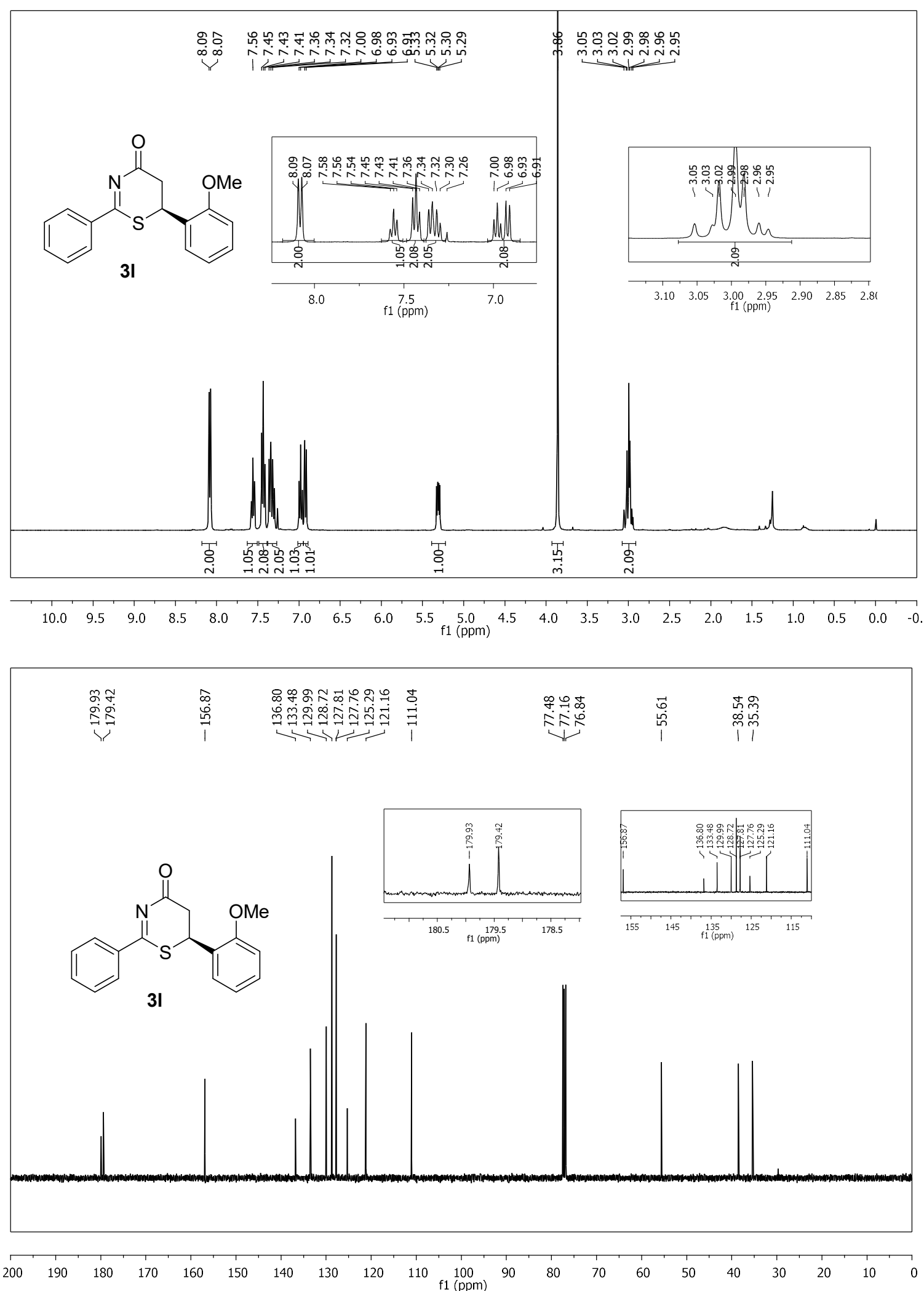
(S)-6-(2-Bromophenyl)-2-phenyl-5,6-dihydro-4H-1,3-thiazin-4-one (3m)
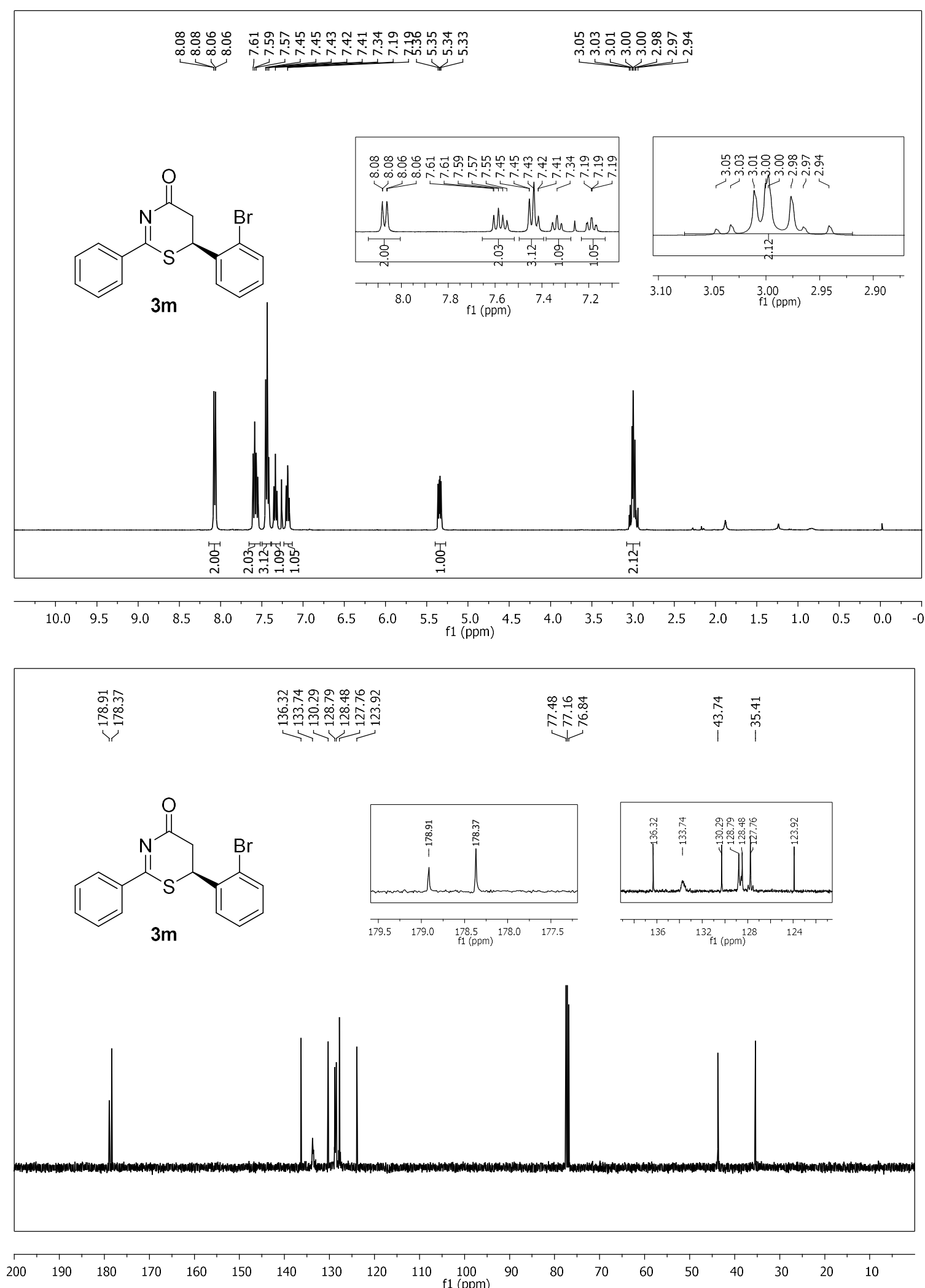
(S)-6-(2-Chlorophenyl)-2-phenyl-5,6-dihydro-4H-1,3-thiazin-4-one (3n)
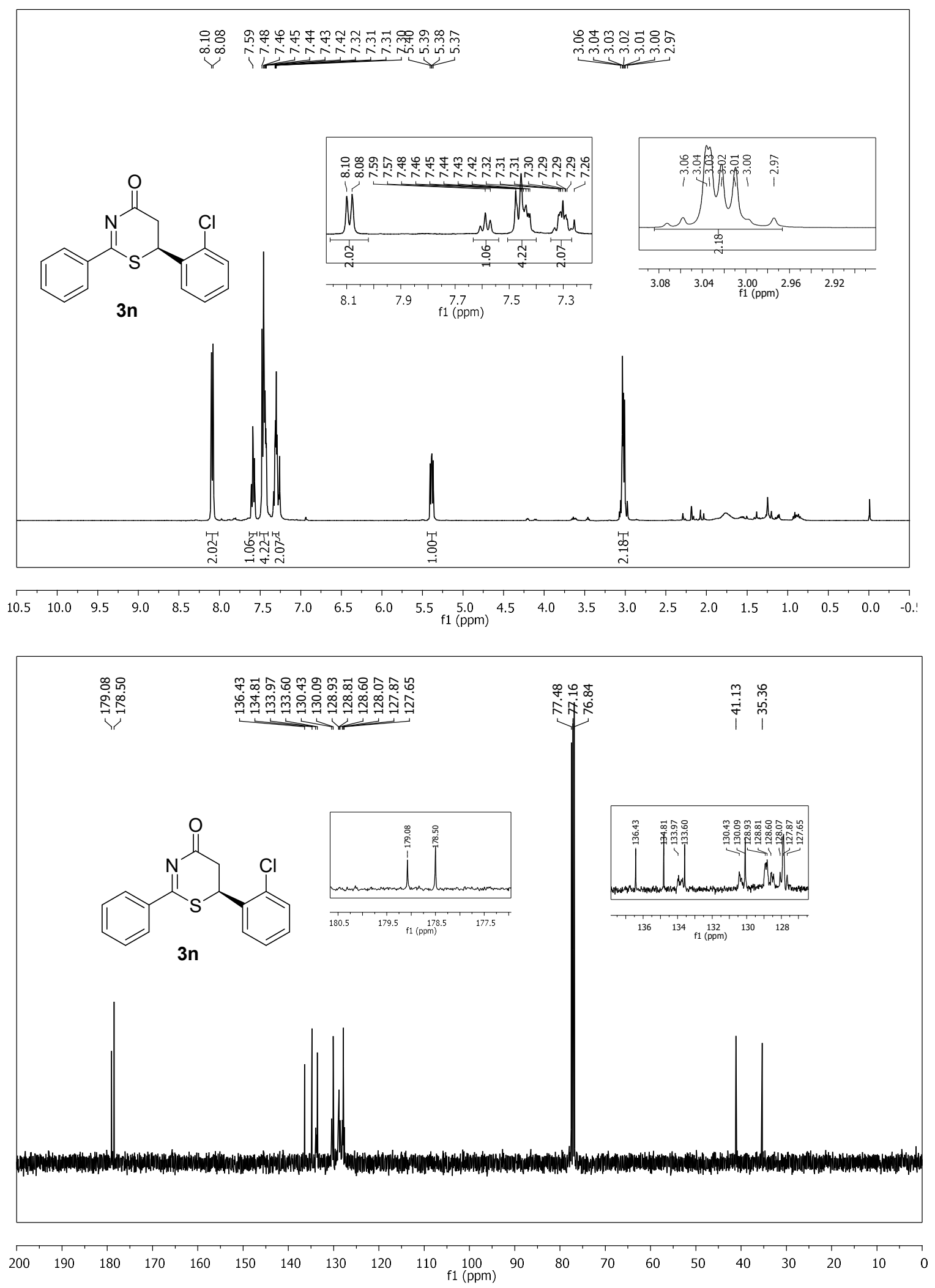
(S)-6-(3,4-Dichlorophenyl)-2-phenyl-5,6-dihydro-4H-1,3-thiazin-4-one (3o)
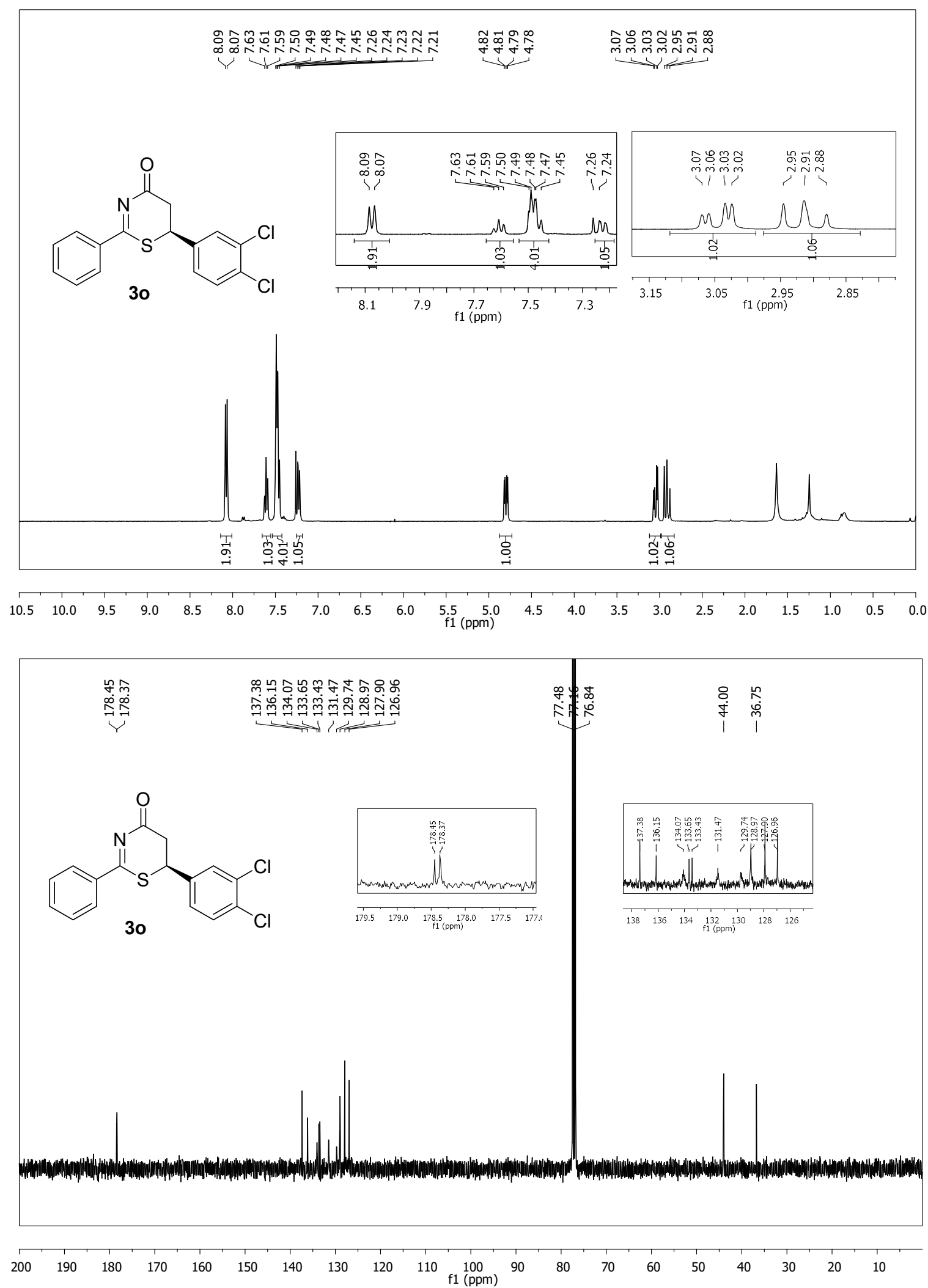
(S)-6-(3-Bromo-4-methoxyphenyl)-2-phenyl-5,6-dihydro-4H-1,3-thiazin-4-one (3p)
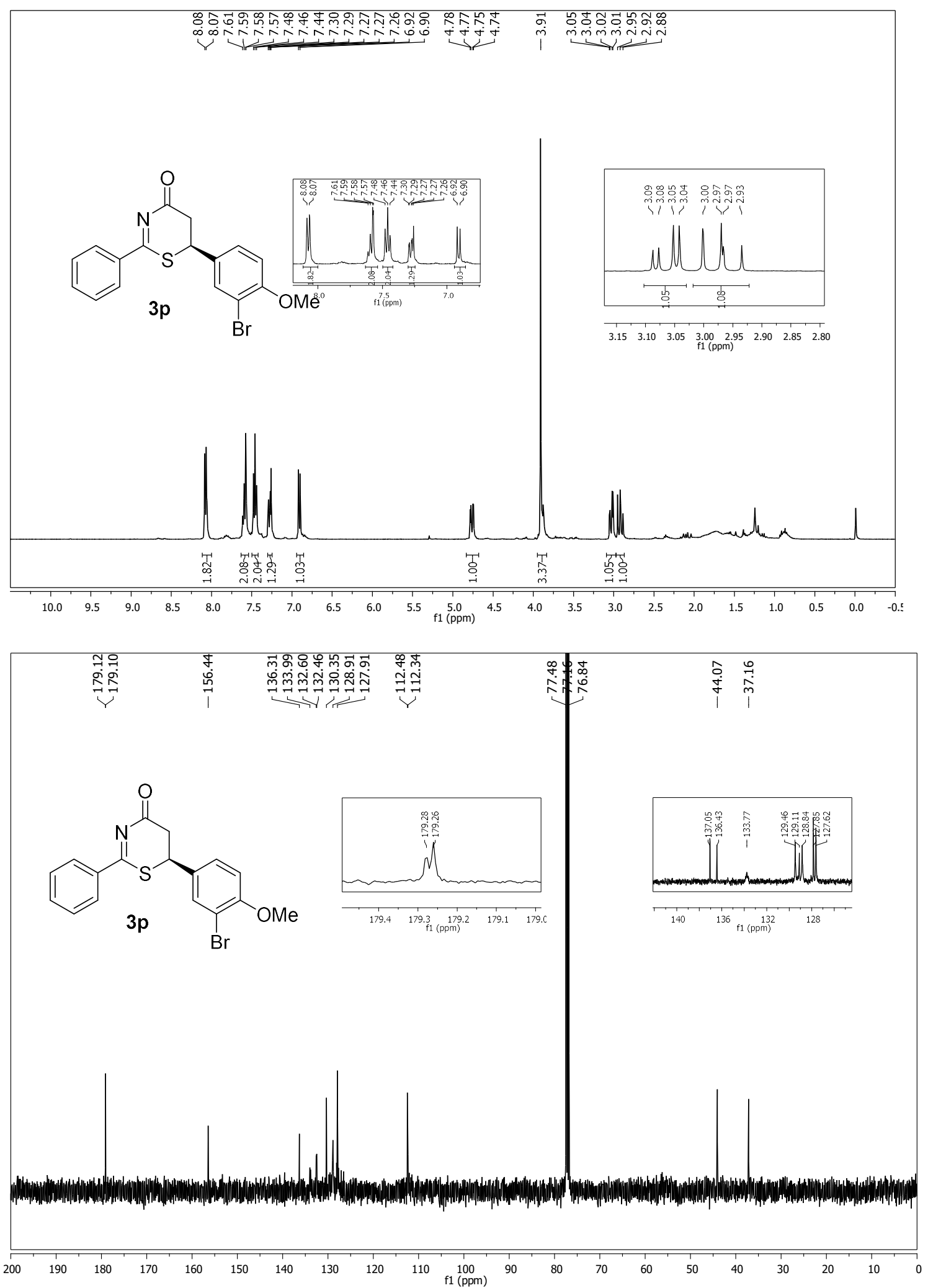
(S)-6-(2-Bromo-4,5-dimethoxyphenyl)-2-phenyl-5,6-dihydro-4H-1,3-thiazin-4-one (3q)
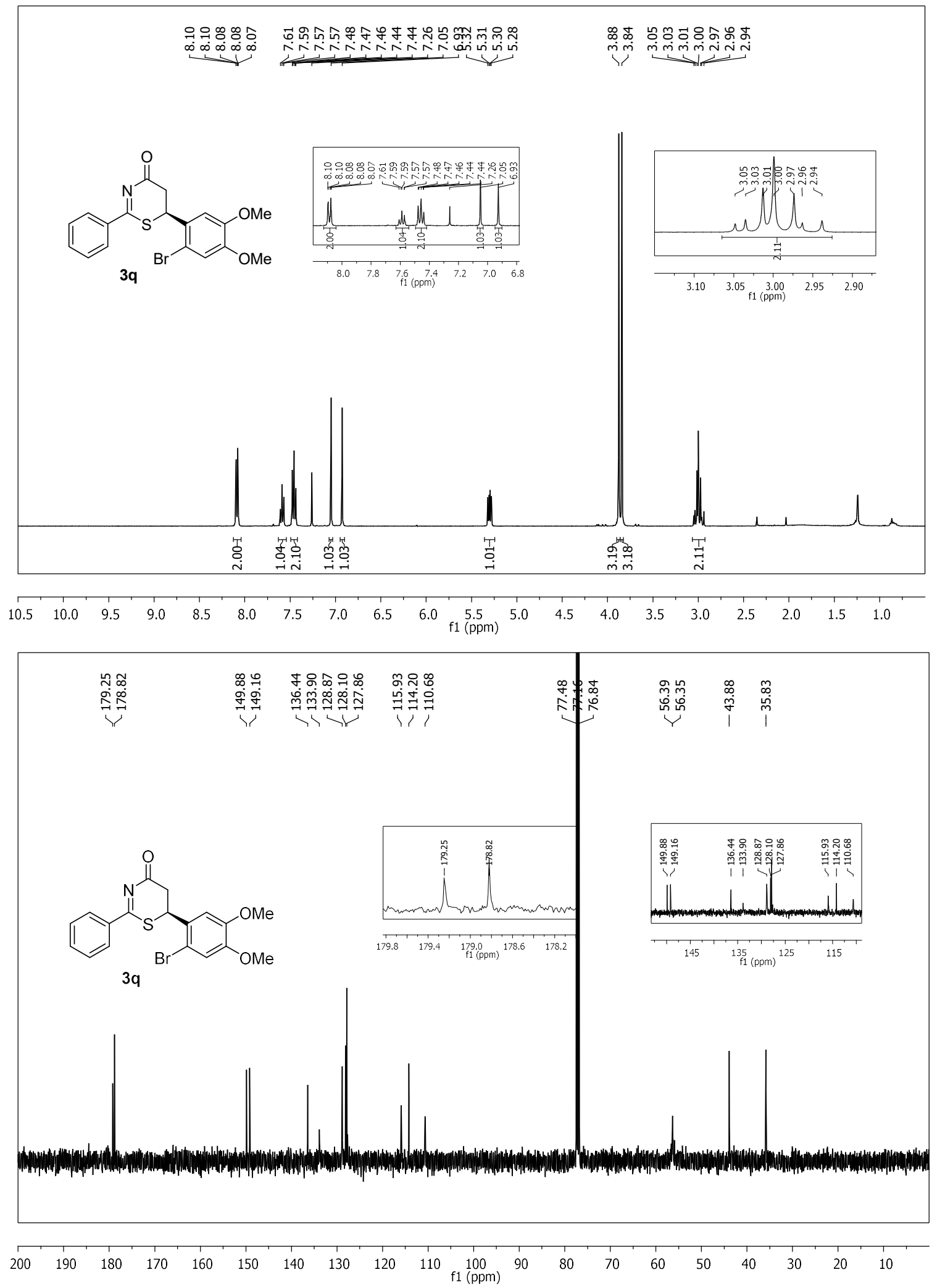
(S)-6-(Furan-2-yl)-2-phenyl-5,6-dihydro-4H-1,3-thiazin-4-one (3r)
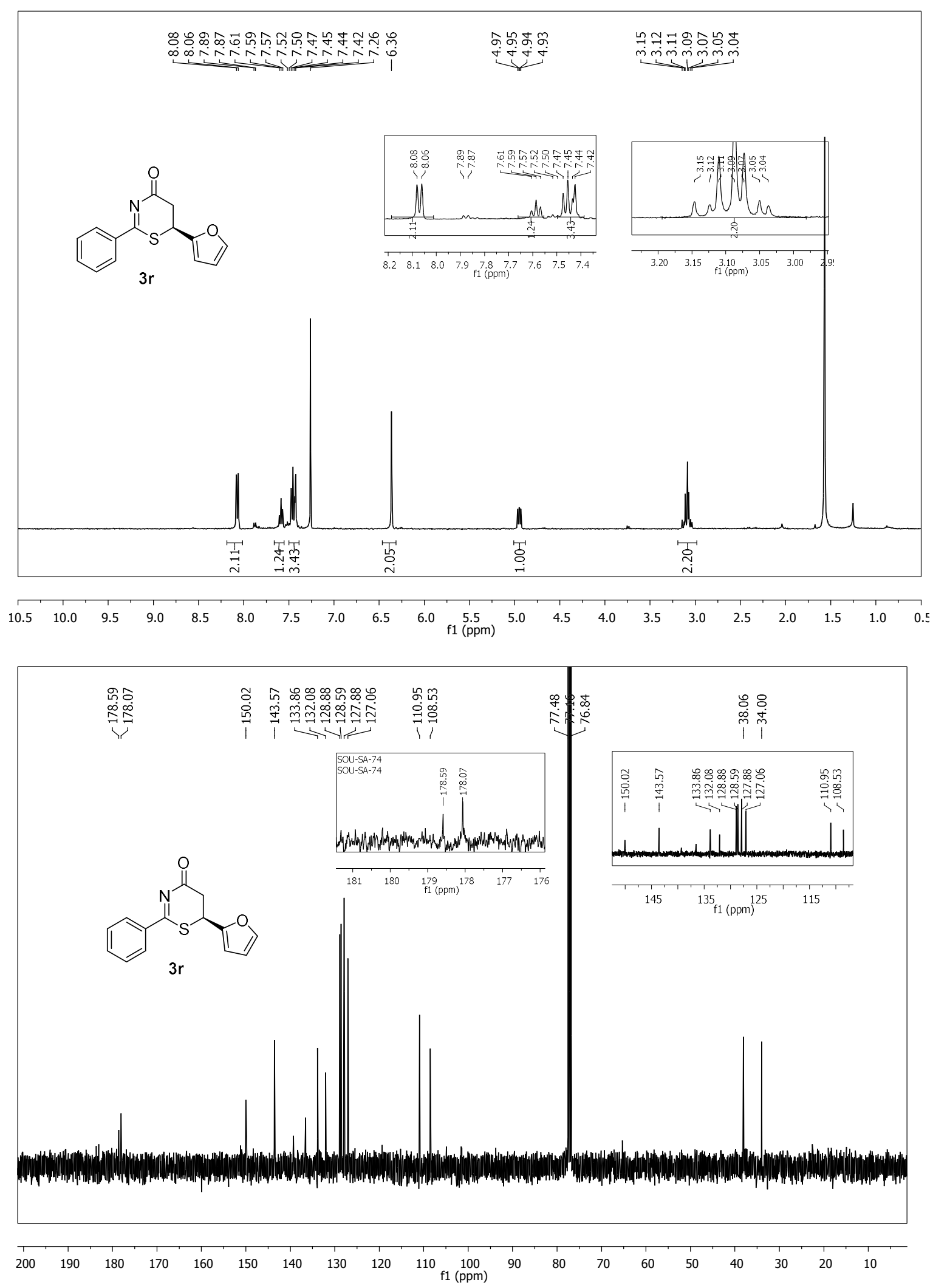
(S)-2-Phenyl-6-(thiophen-2-yl)-5,6-dihydro-4H-1,3-thiazin-4-one (3s)
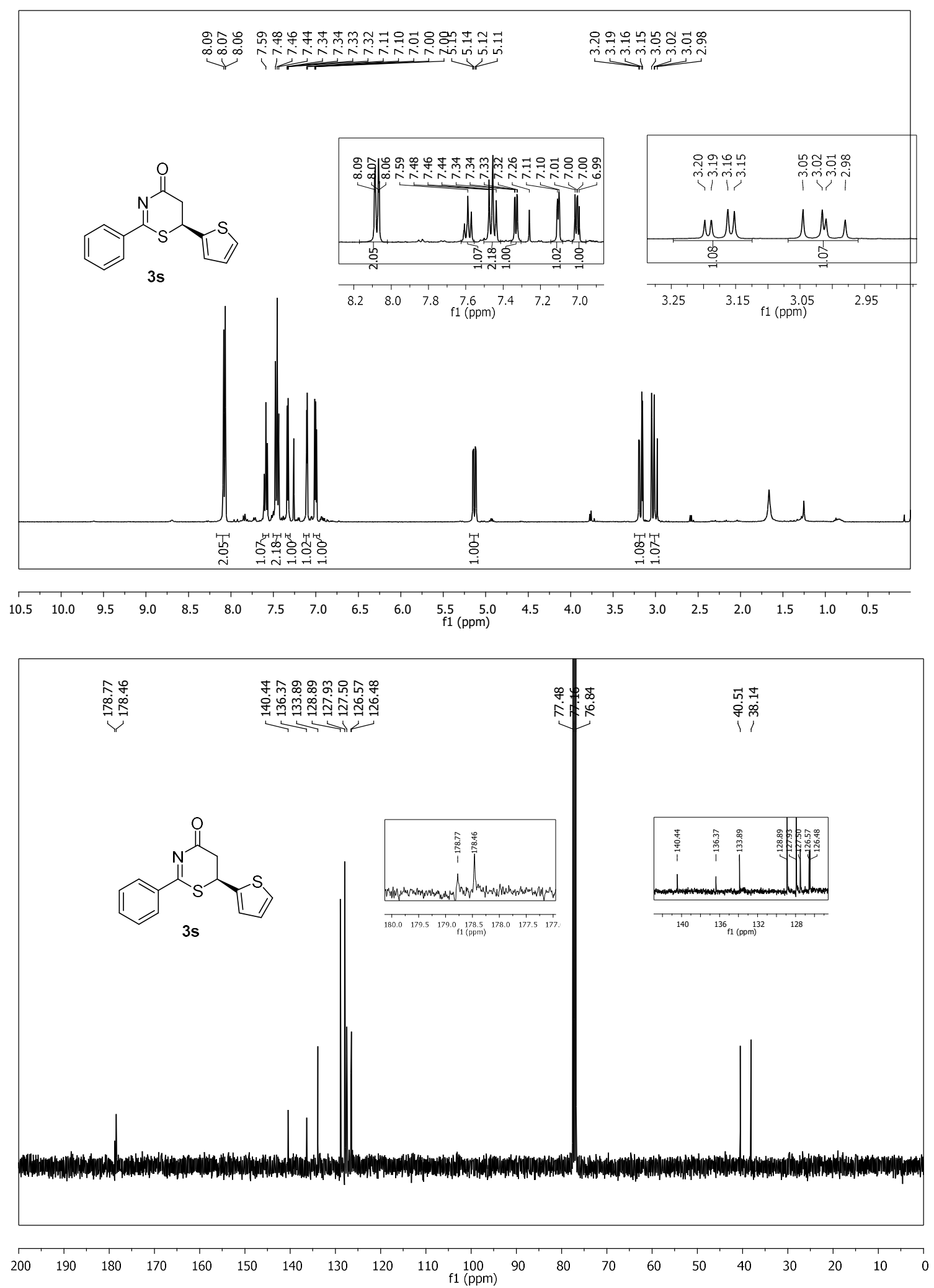
(R)-6-Methyl-2-phenyl-5,6-dihydro-4H-1,3-thiazin-4-one (3t)
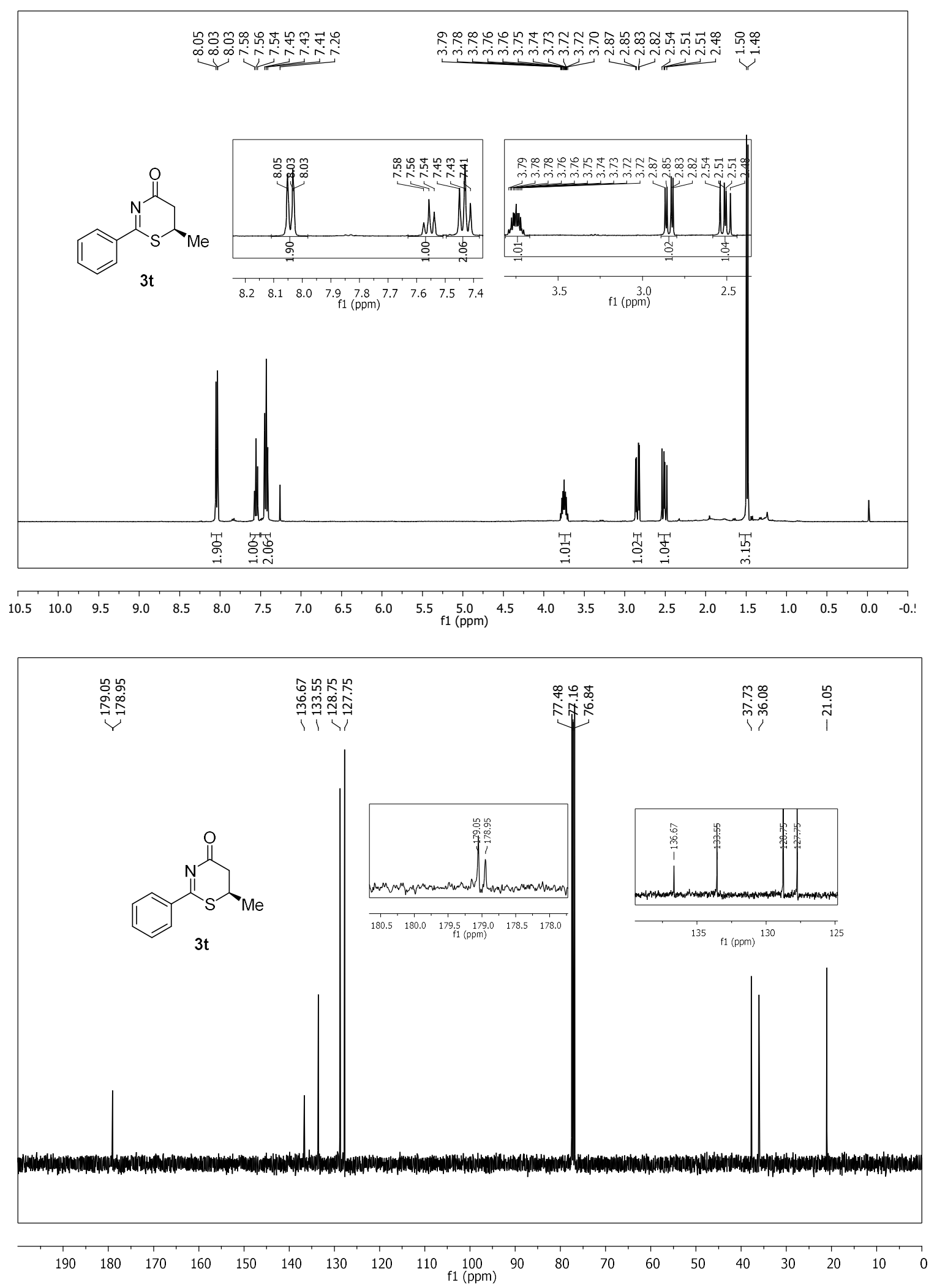
(R)-6-Ethyl-2-phenyl-5,6-dihydro-4H-1,3-thiazin-4-one(3u)
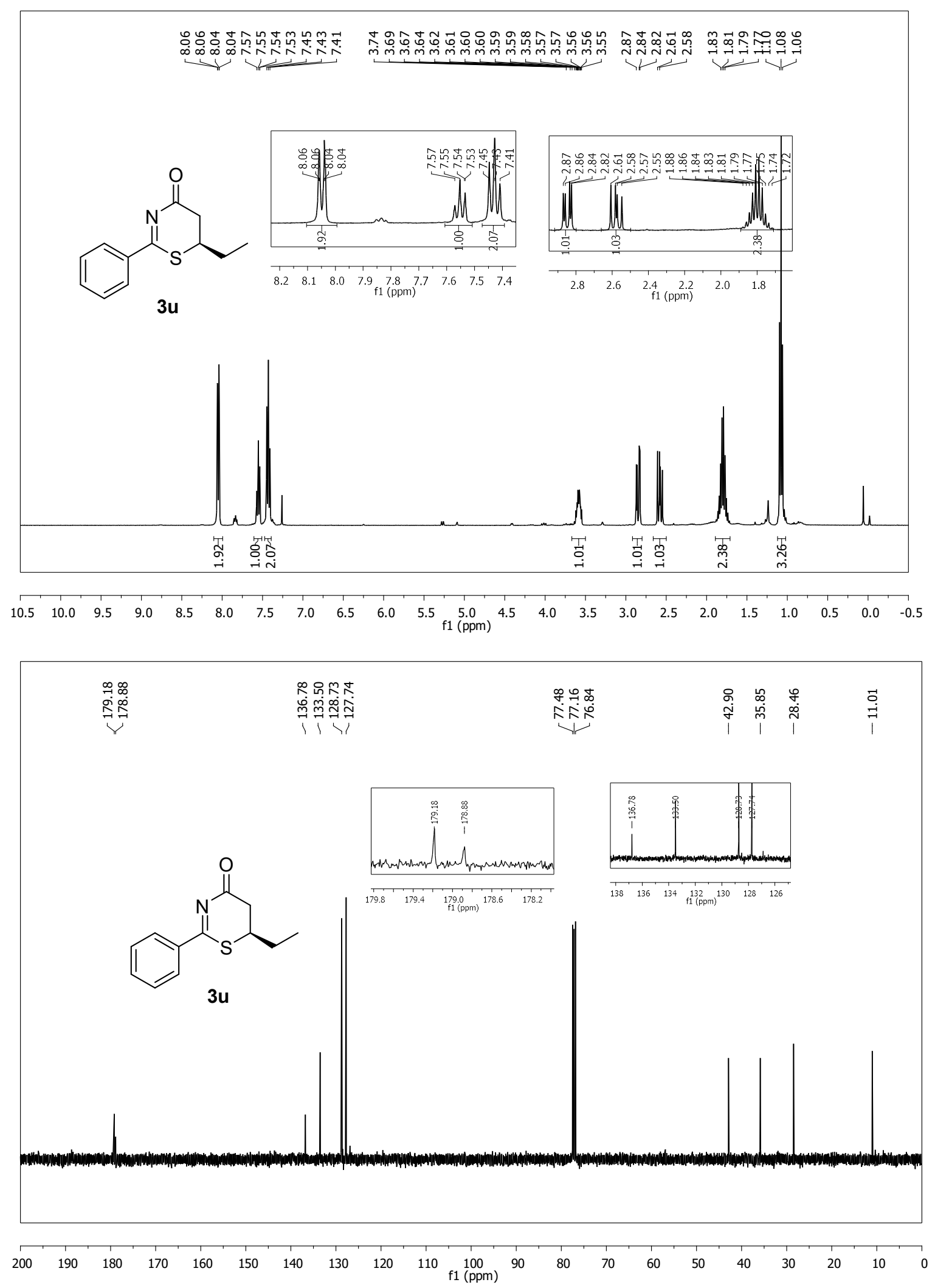
(S)-2-(4-Methoxyphenyl)-6-phenyl-5,6-dihydro-4H-1,3-thiazin-4-one (3v)
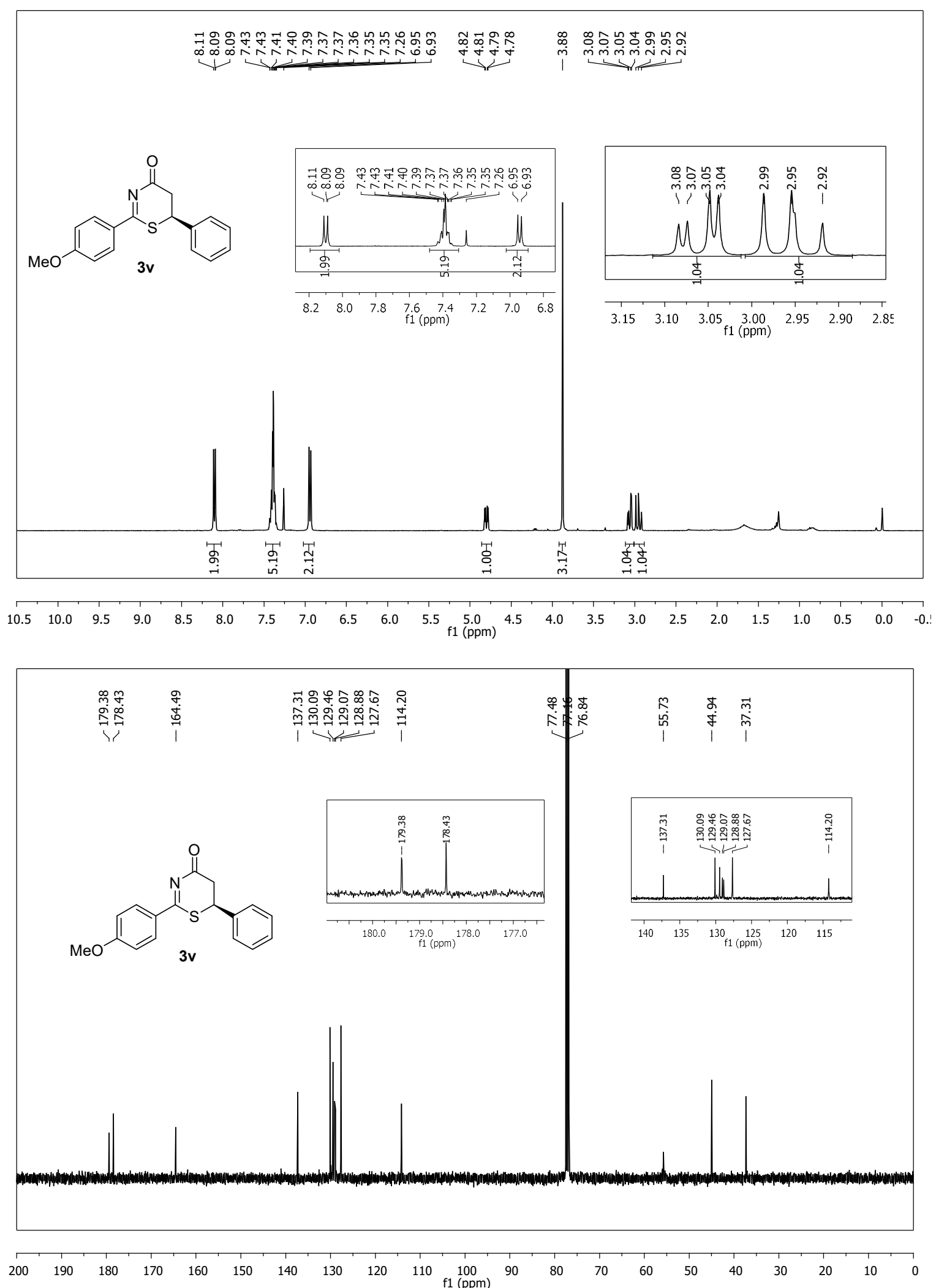
(S)-6-Phenyl-2-(p-tolyl)-5,6-dihydro-4H-1,3-thiazin-4-one (3w)
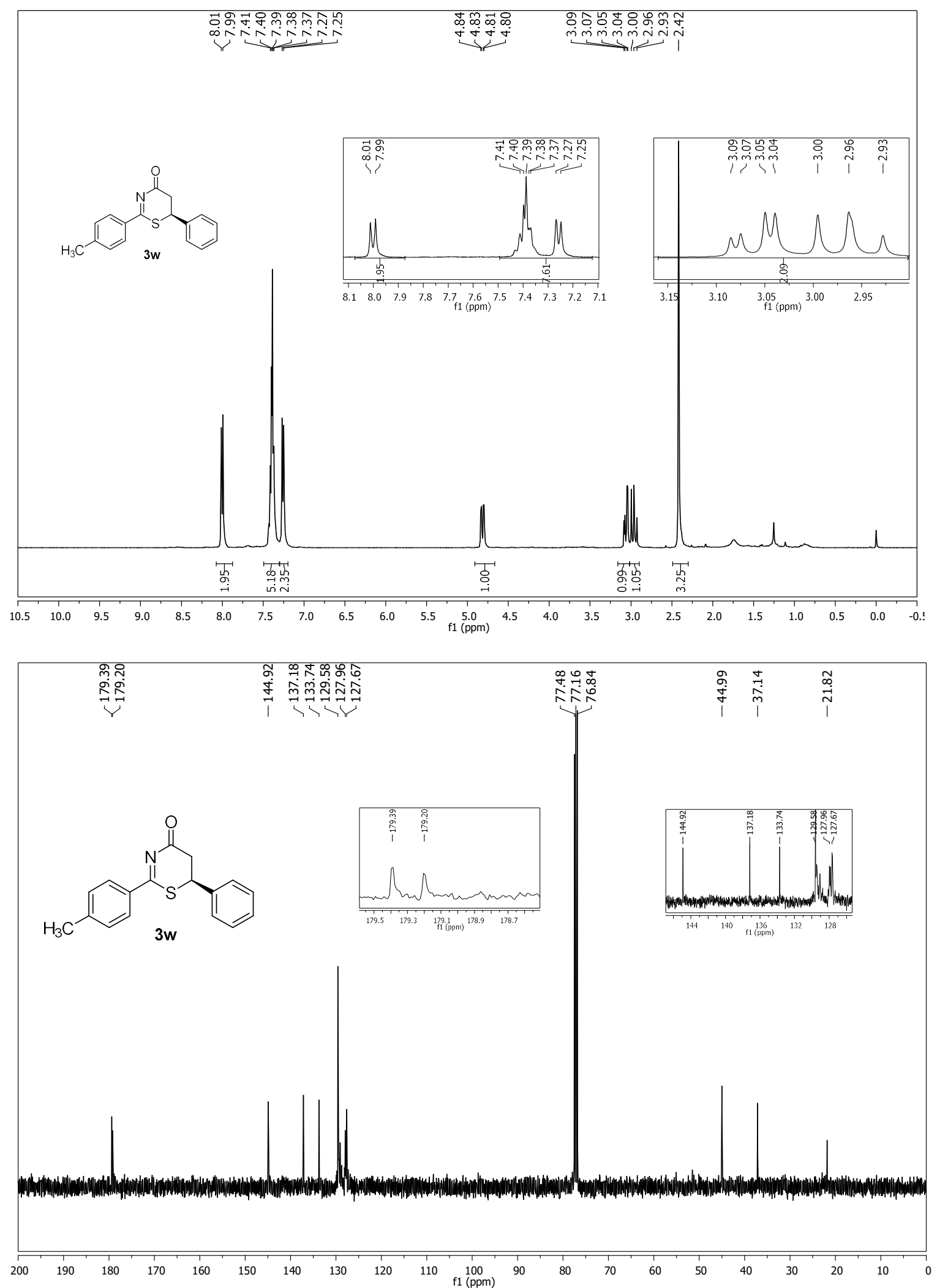
(S)-2-(4-Iodophenyl)-6-phenyl-5,6-dihydro-4H-1,3-thiazin-4-one (3x)
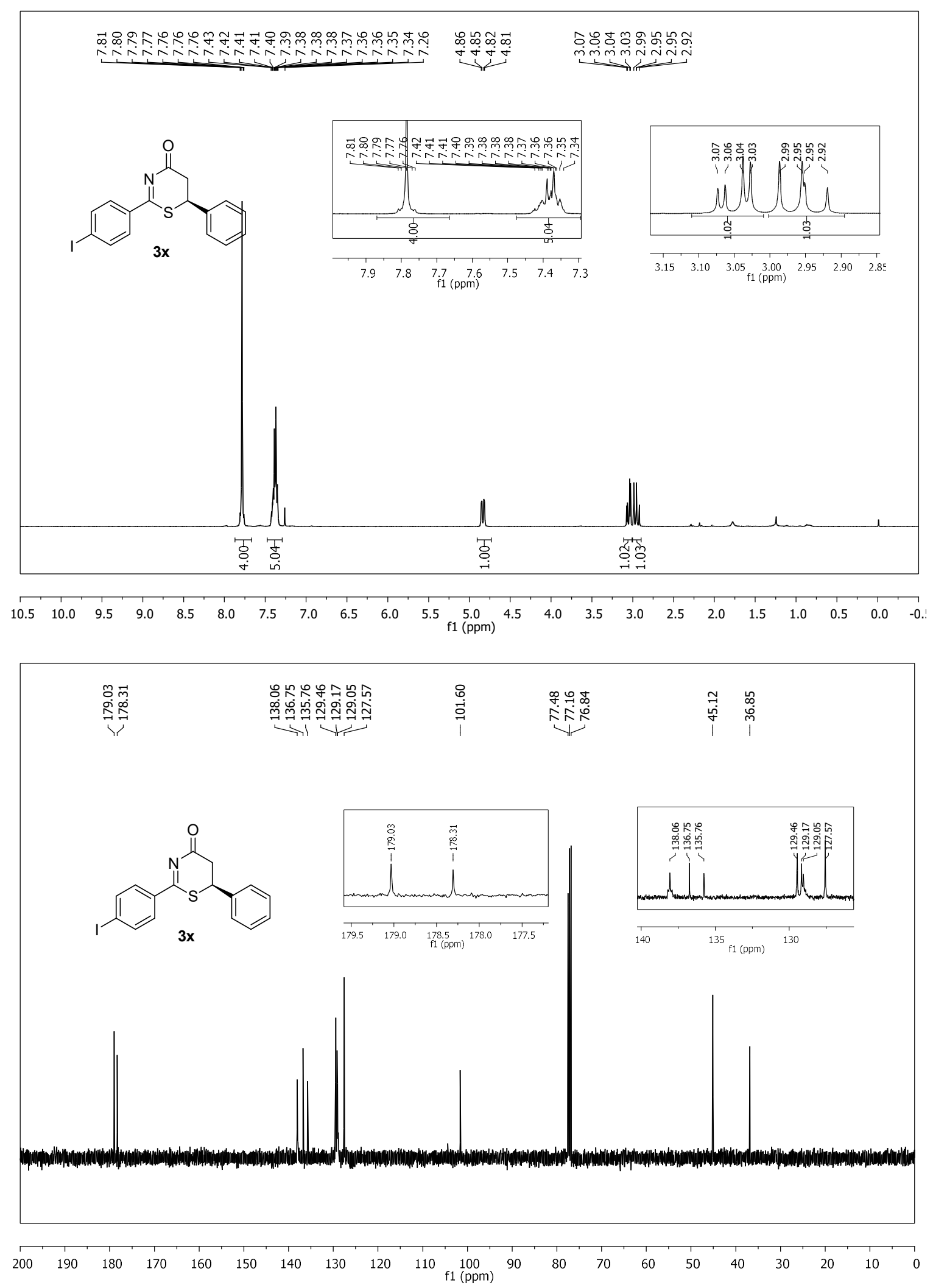
(S)-2-(4-Bromophenyl)-6-phenyl-5,6-dihydro-4H-1,3-thiazin-4-one (3y)
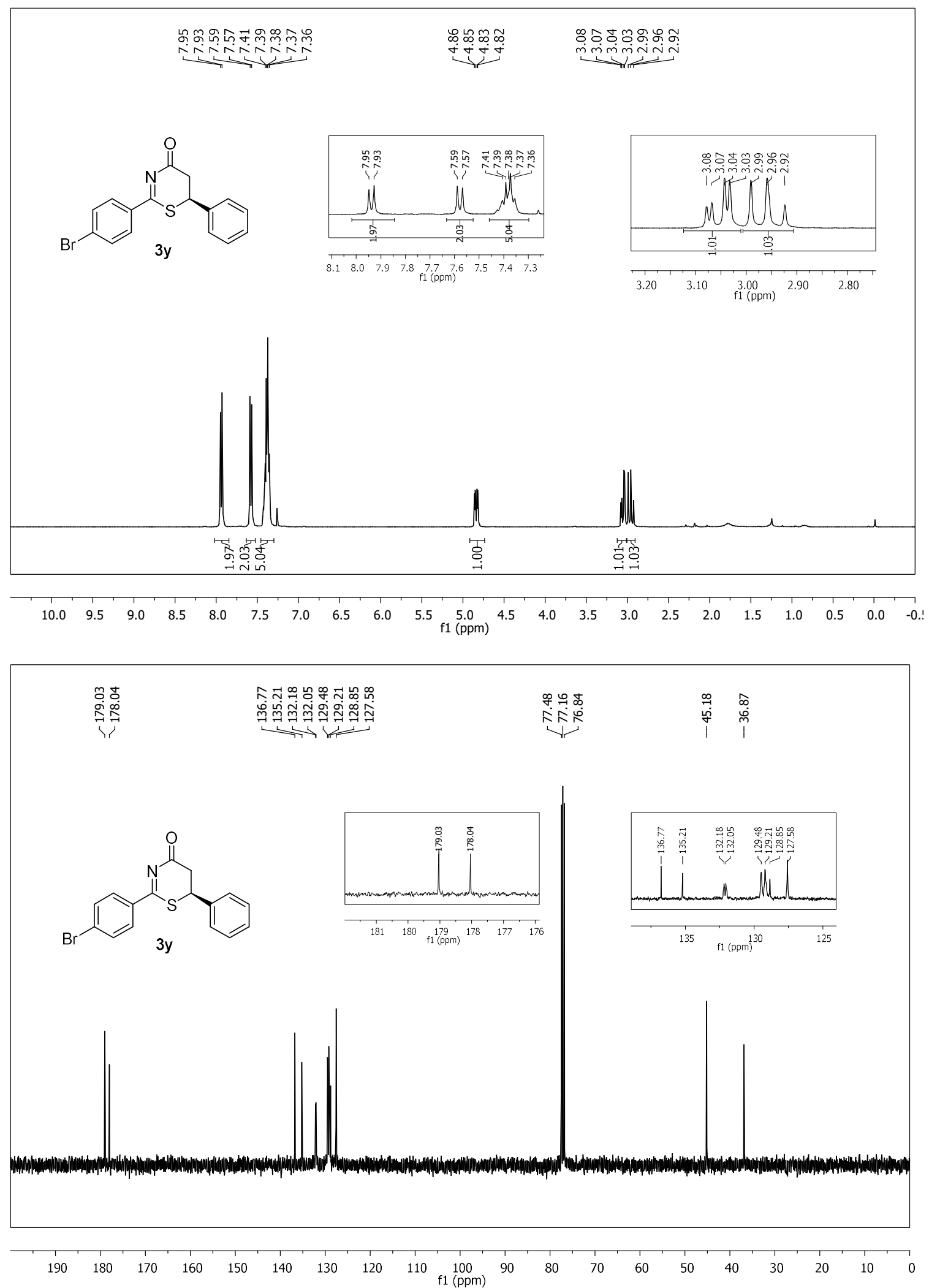
(S)-2-(3-Methoxyphenyl)-6-phenyl-5,6-dihydro-4H-1,3-thiazin-4-one (3z)
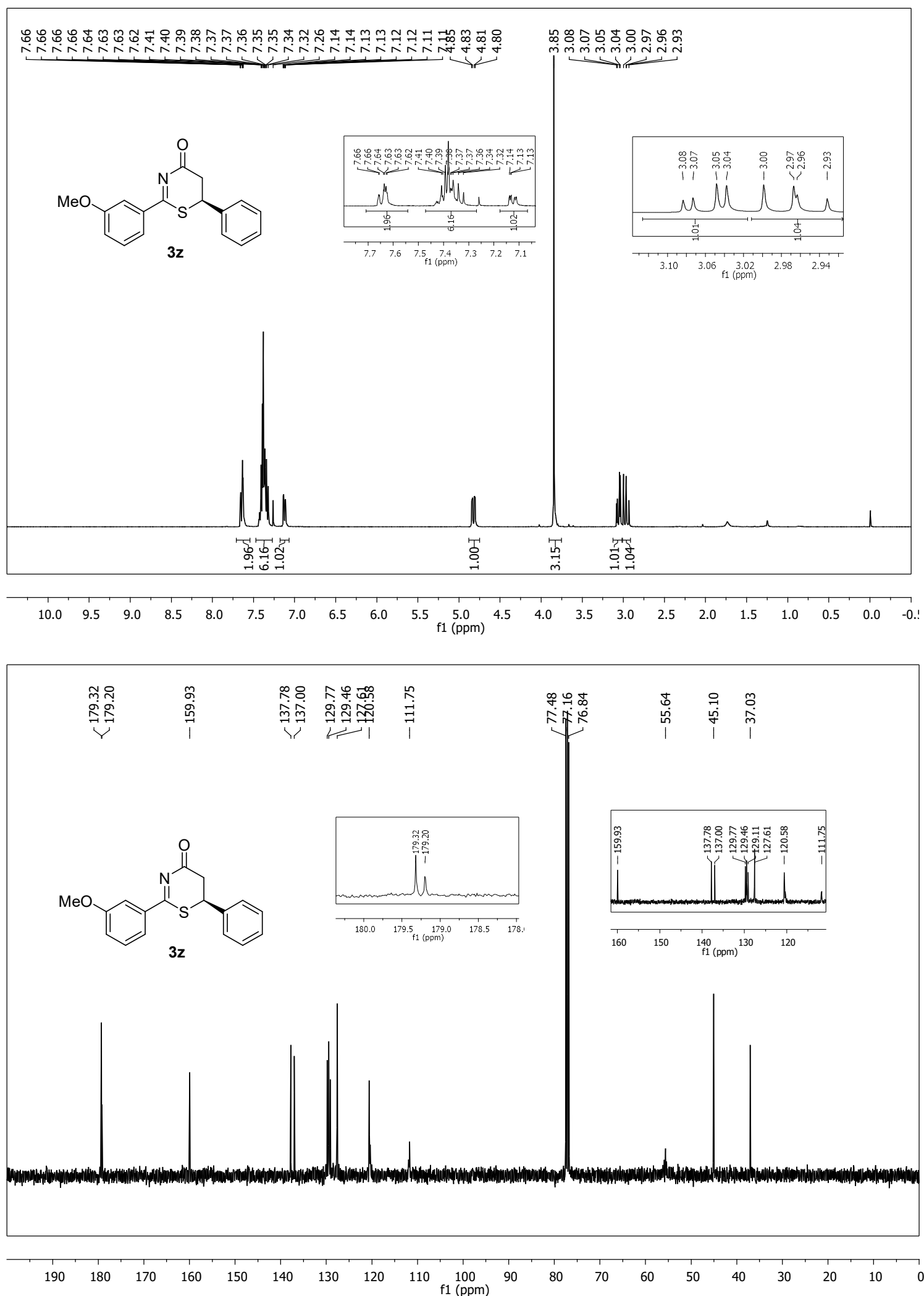
(S)-6-Phenyl-2-(m-tolyl)-5,6-dihydro-4H-1,3-thiazin-4-one (3aa)
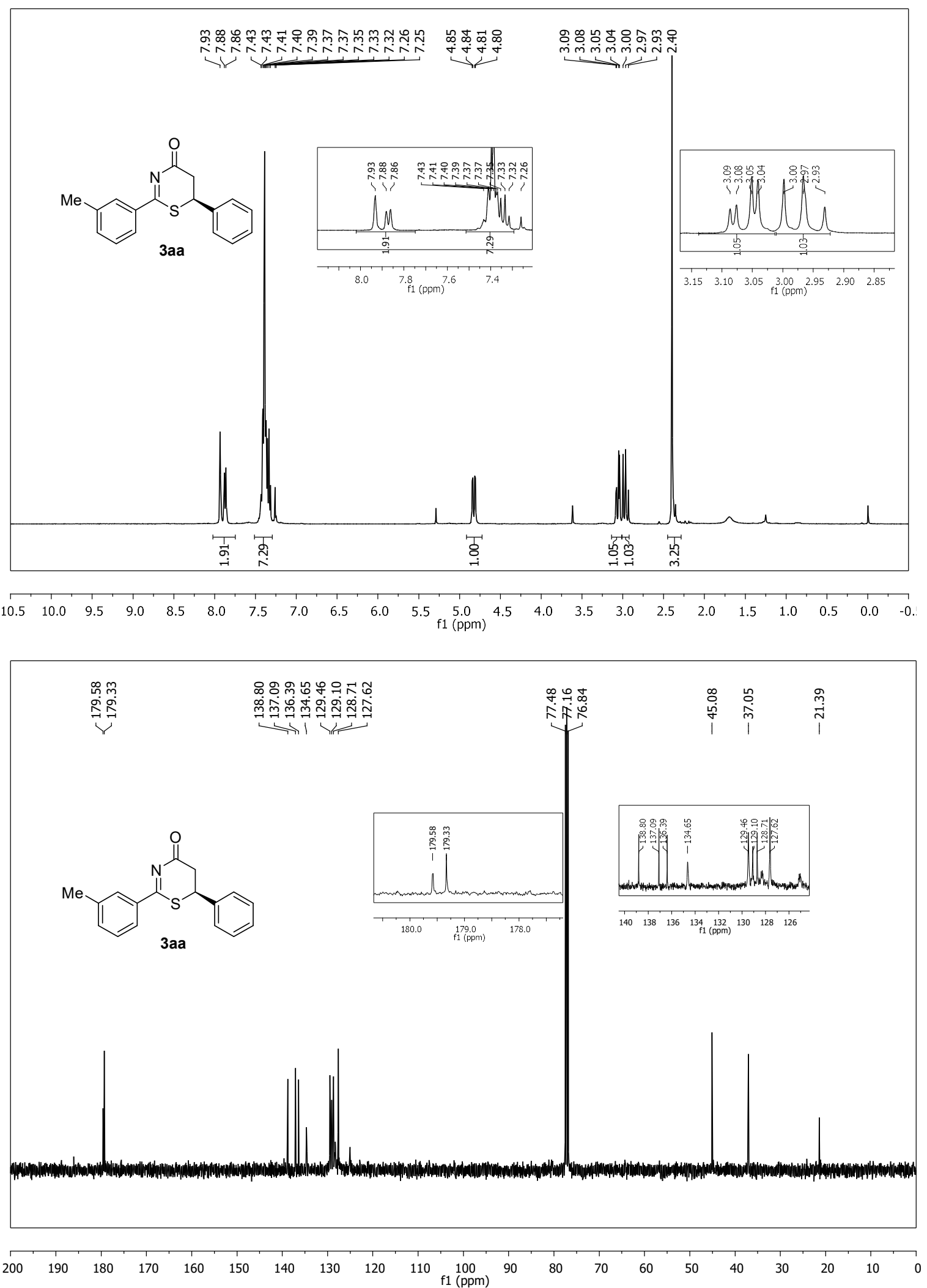
(S)-2-(3-Bromophenyl)-6-phenyl-5,6-dihydro-4H-1,3-thiazin-4-one (3ab)
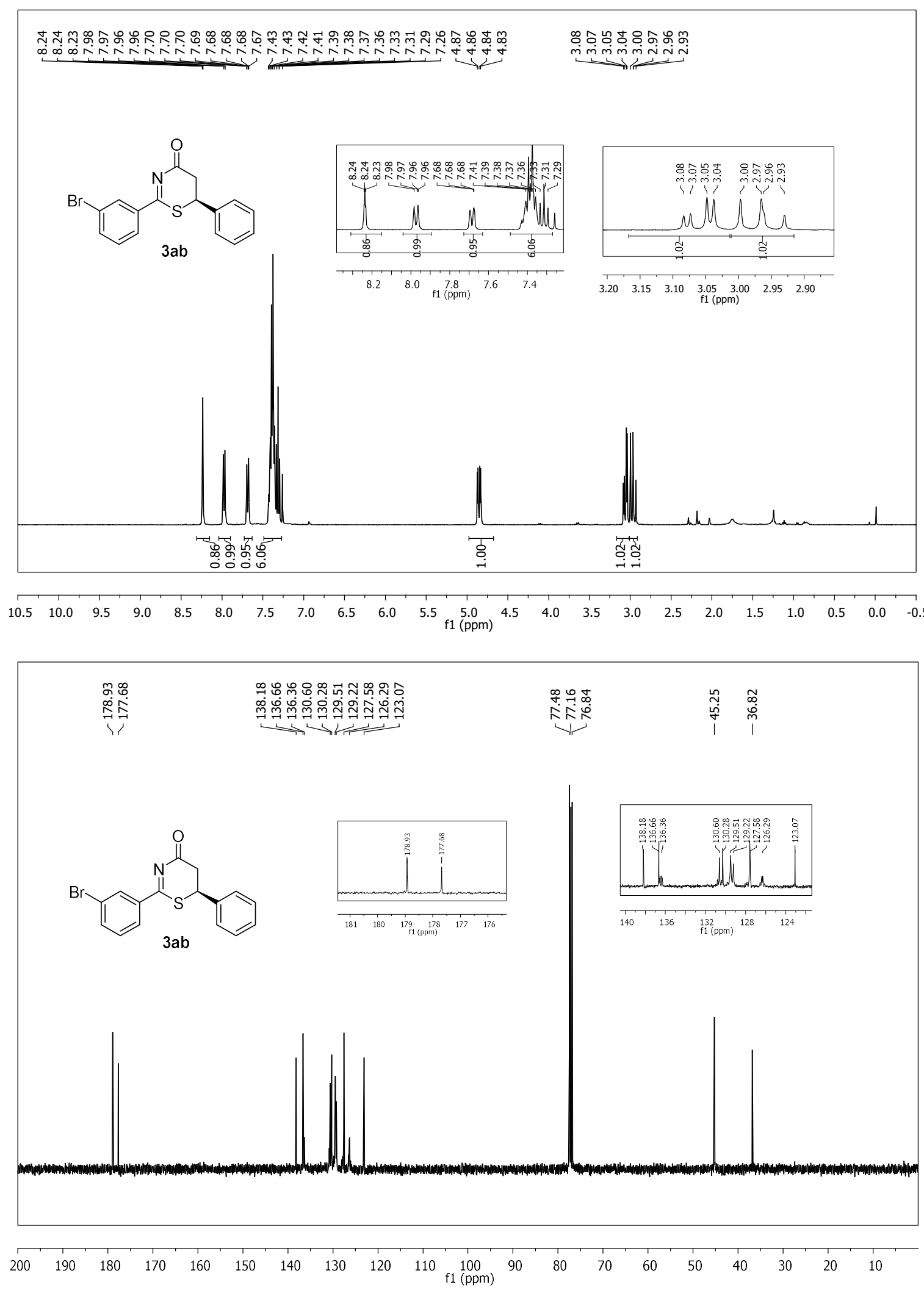
(S)-2-(3-Nitrophenyl)-6-phenyl-5,6-dihydro-4H-1,3-thiazin-4-one (3ac)
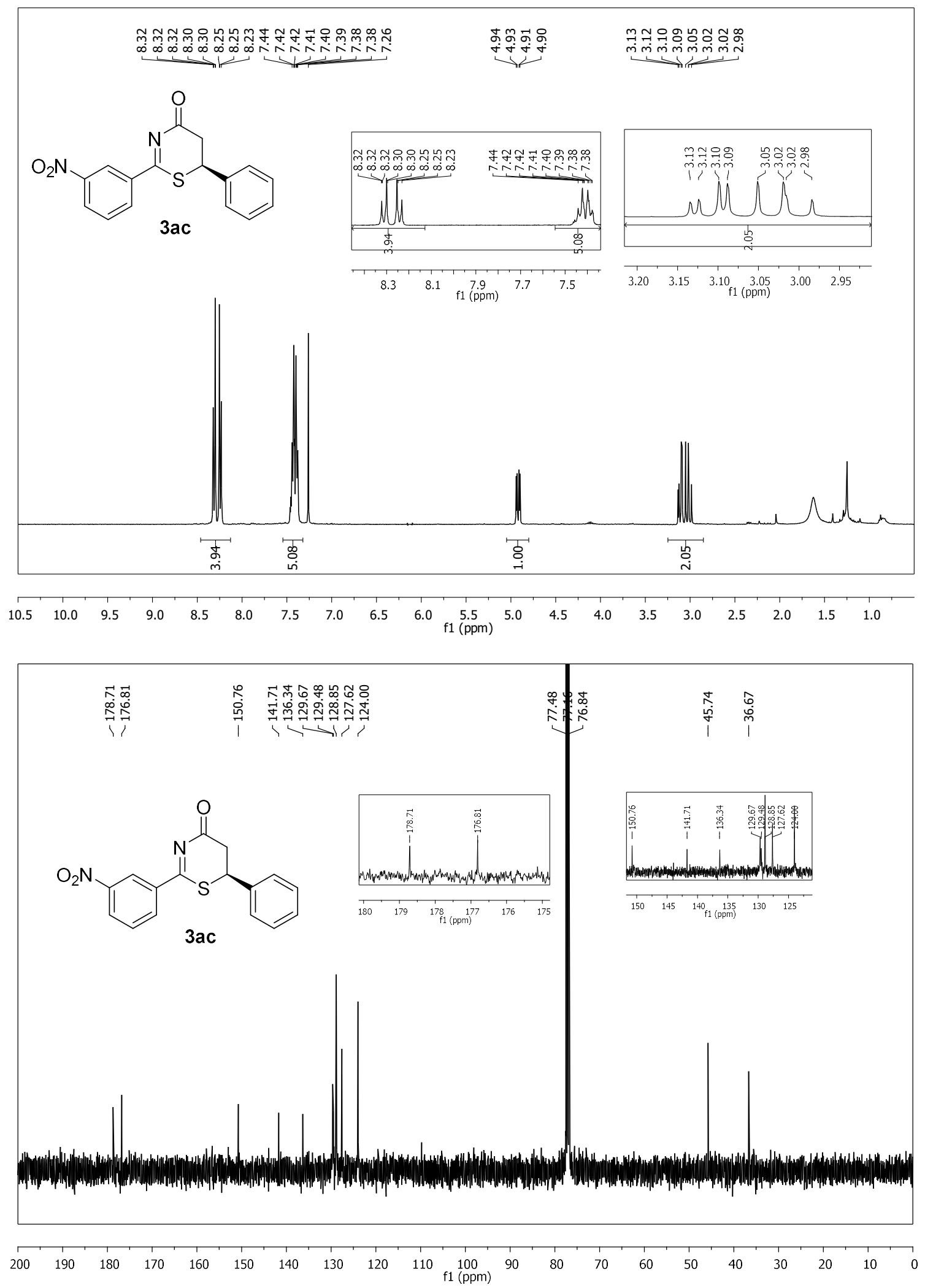
(S)-2-(2-Fluorophenyl)-6-phenyl-5,6-dihydro-4H-1,3-thiazin-4-one (3ad)
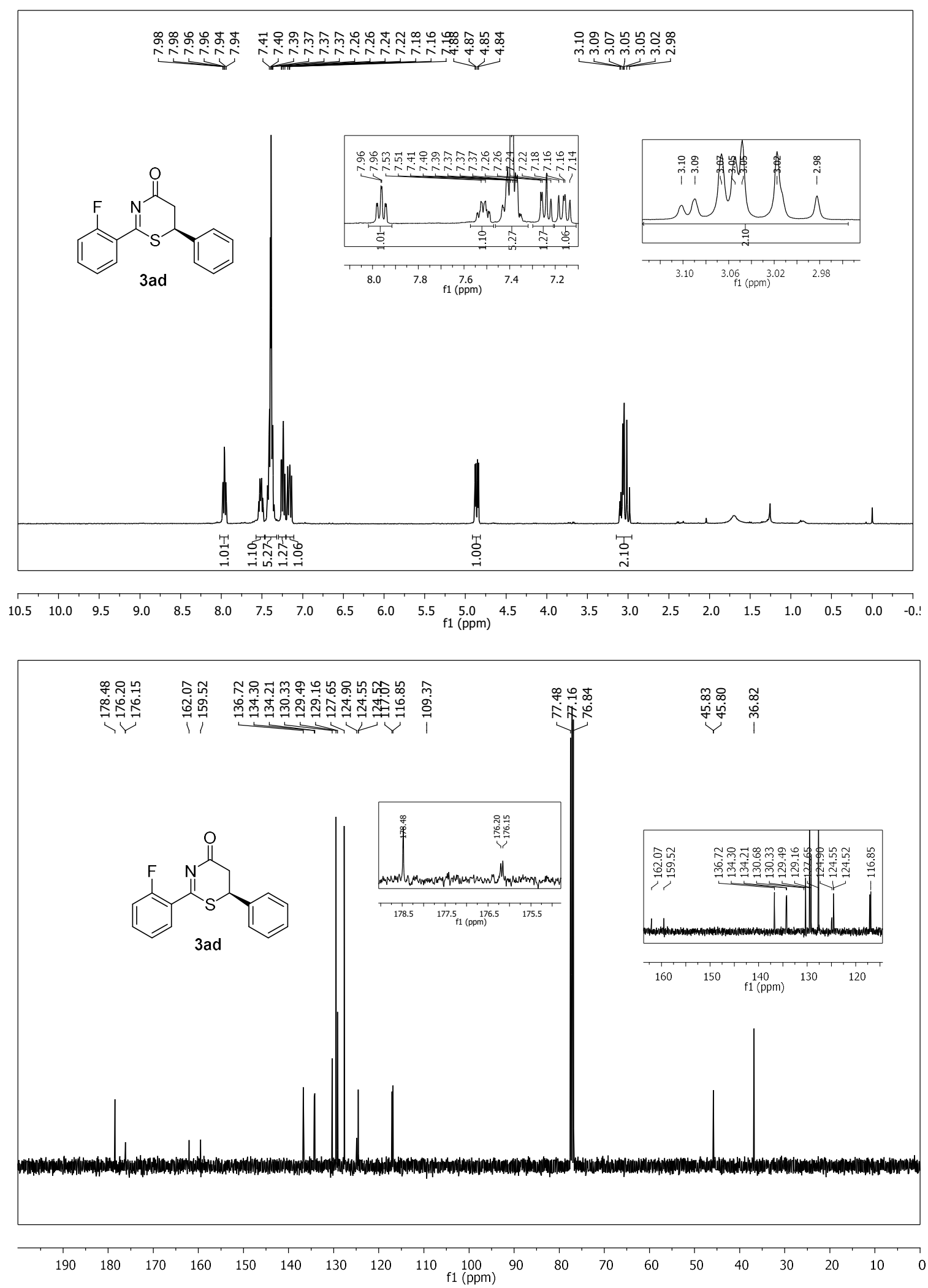
(S)-2-(Naphthalen-1-yl)-6-phenyl-5,6-dihydro-4H-1,3-thiazin-4-one (3ae)
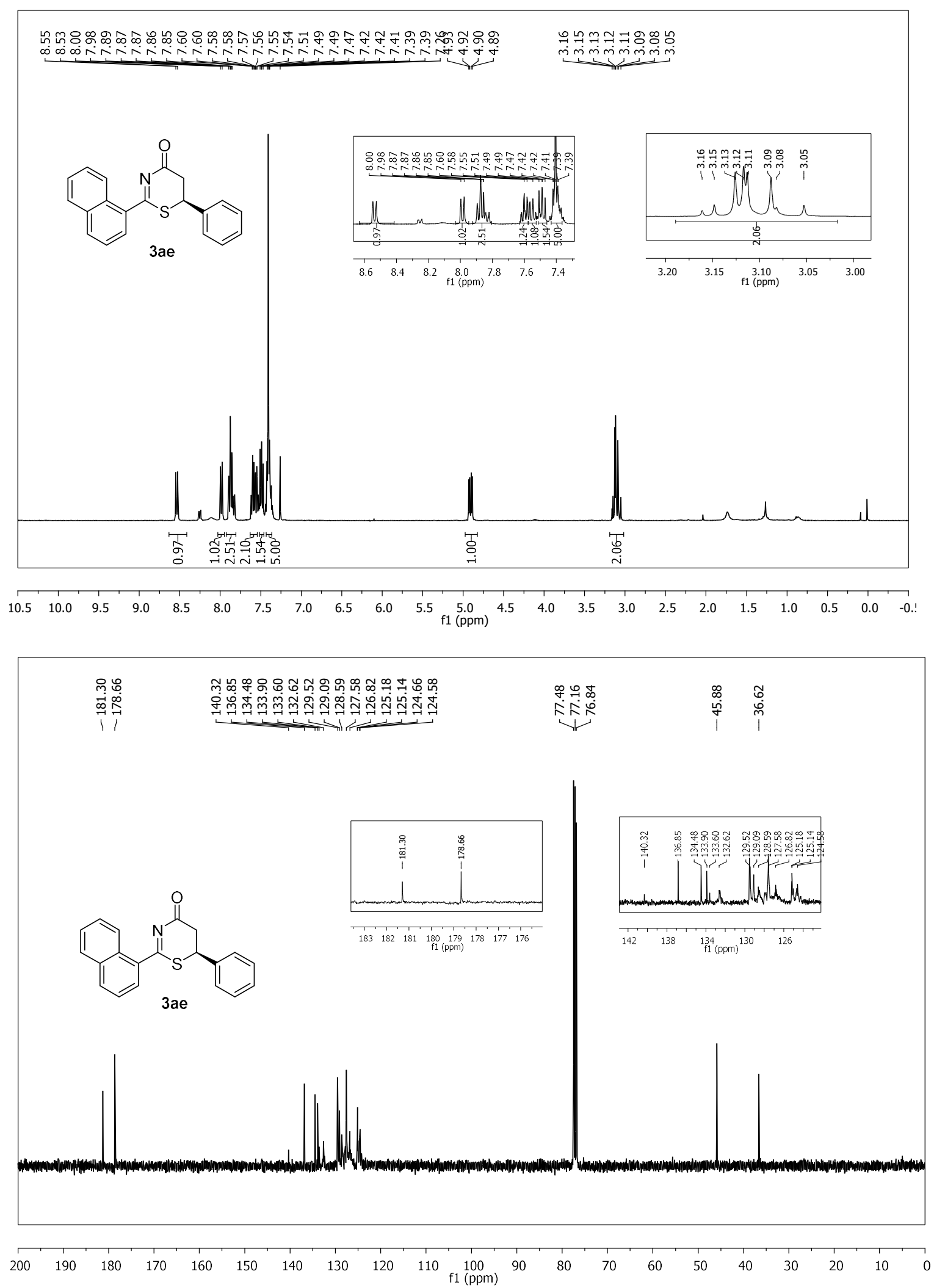
(S)-6-Phenyl-2-(thiophen-2-yl)-5,6-dihydro-4H-1,3-thiazin-4-one (3af)
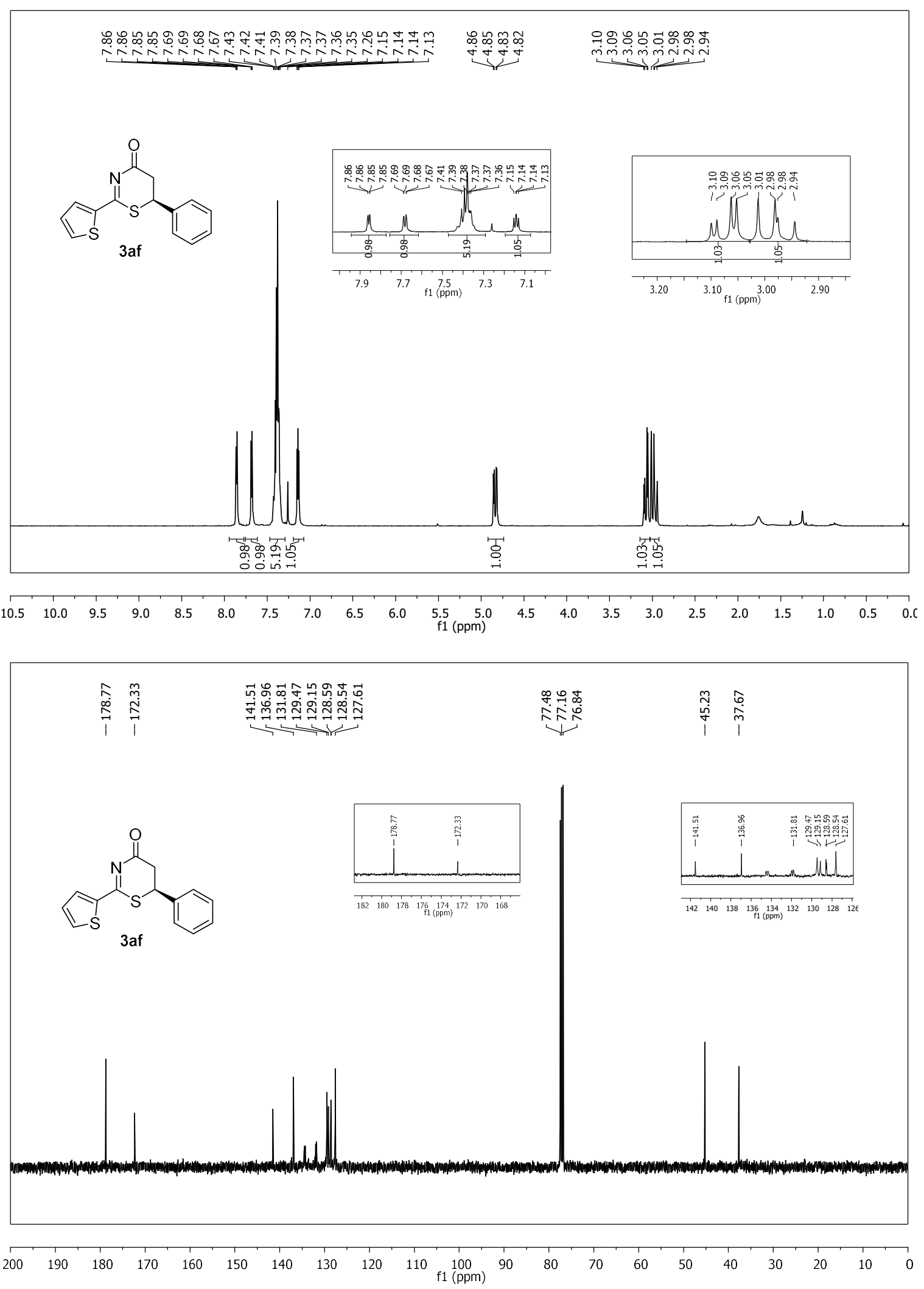


\section{HPLC Data of Functionalized Thiazinones}

(S)-2,6-Diphenyl-5,6-dihydro-4H-1,3-thiazin-4-one (3a)
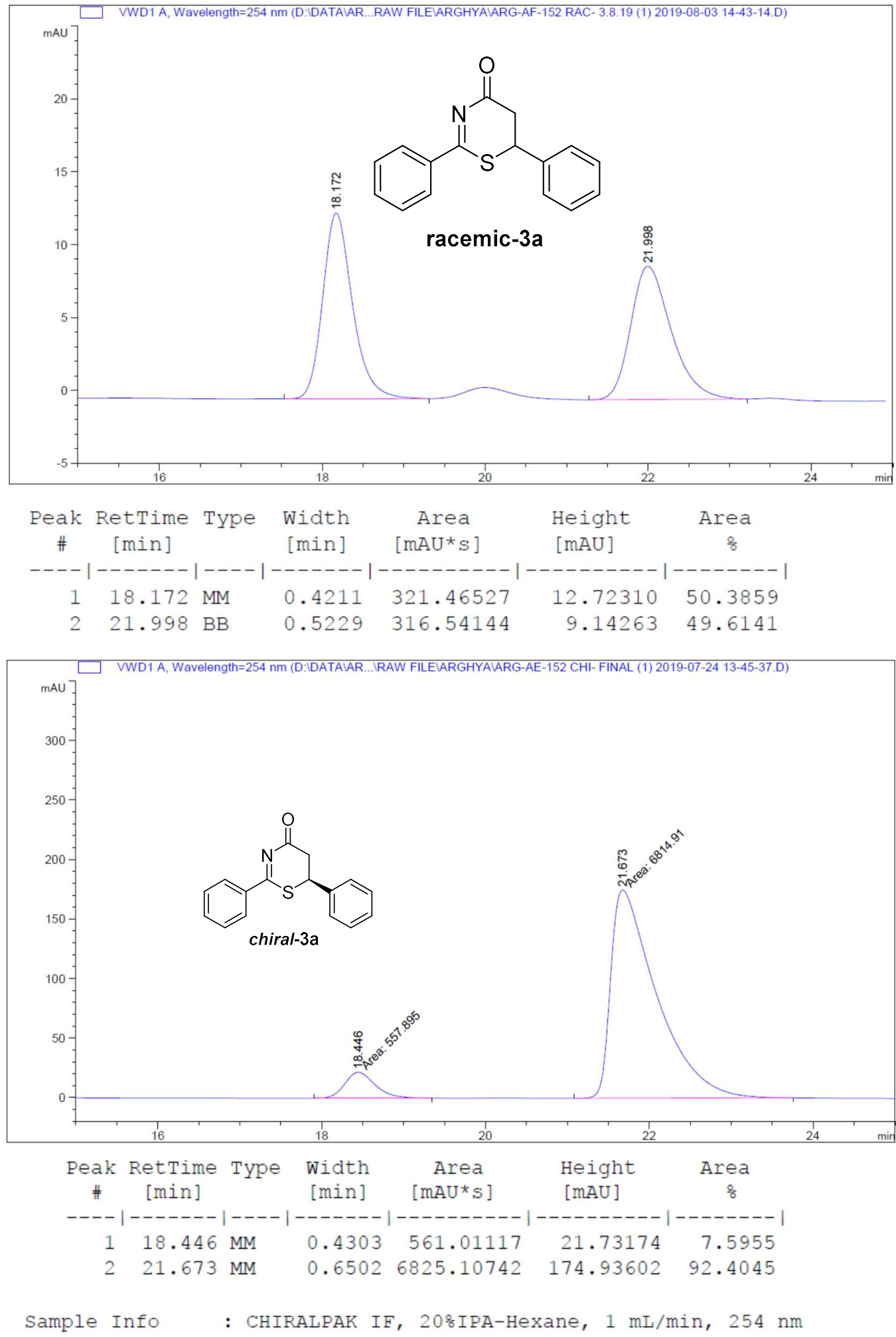
(S)-6-(4-Methoxyphenyl)-2-phenyl-5,6-dihydro-4H-1,3-thiazin-4-one (3b)
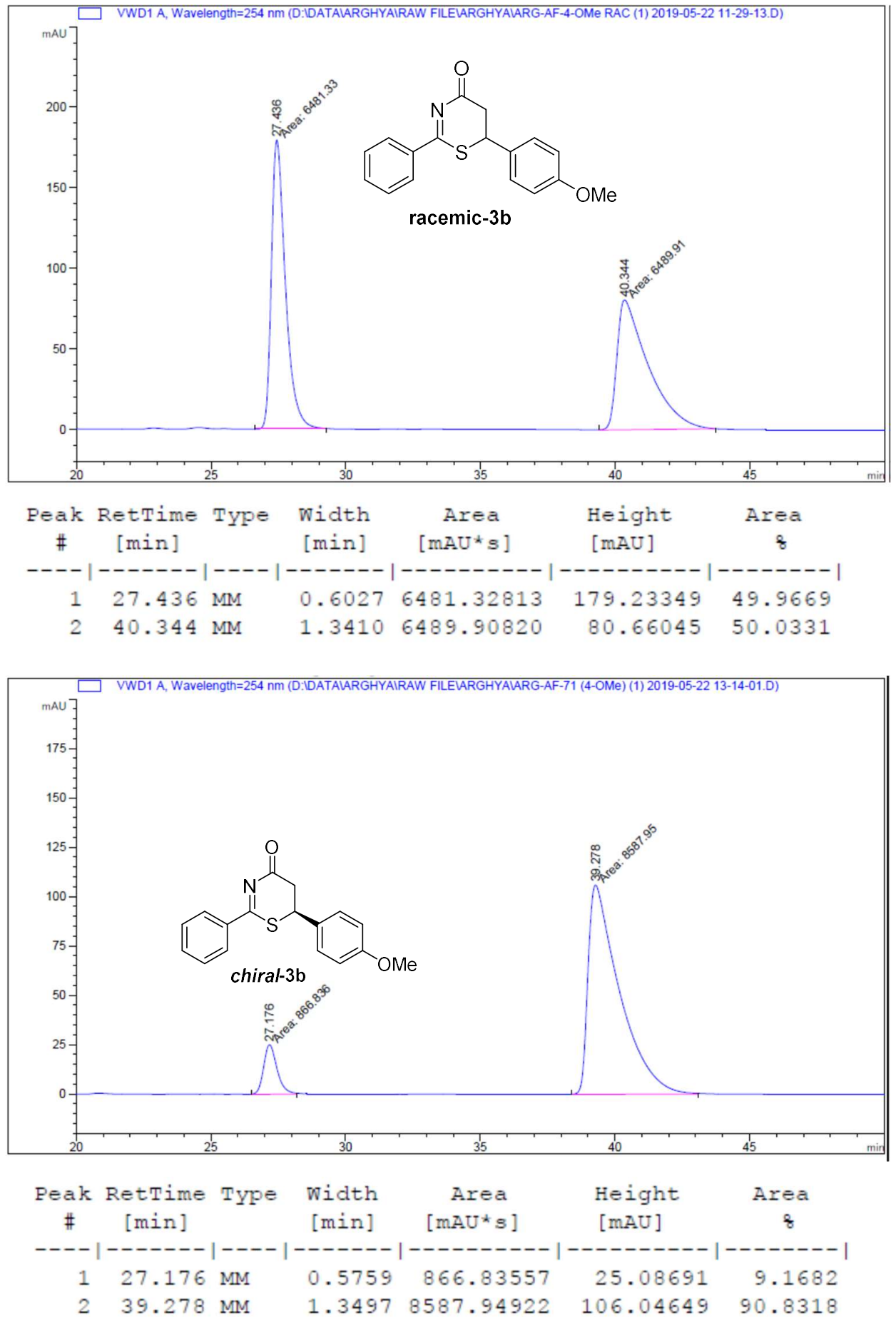

Sample Info: CHIRALPAK IF, 258 IPA-Hexane, $.7 \mathrm{~mL} / \mathrm{min}, 254 \mathrm{~nm}$ 


\section{(S)-2-Phenyl-6-(p-tolyl)-5,6-dihydro-4H-1,3-thiazin-4-one (3c)}
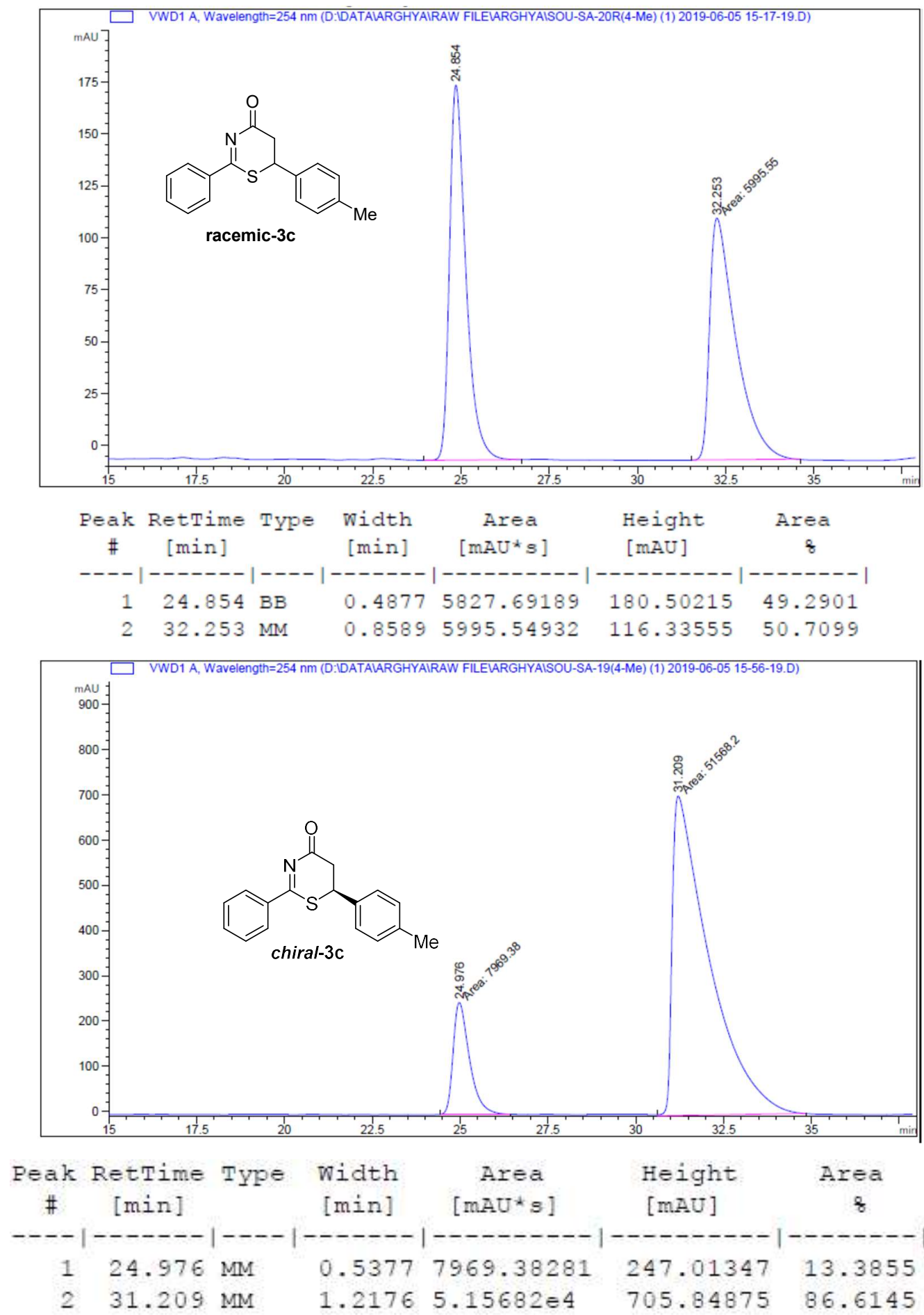

Sample Info : CHIRALPAR IF, 208 IPA-Hexane, $.7 \mathrm{~mL} / \mathrm{min}, 254 \mathrm{~nm}$ 


\section{(S)-6-(4-Bromophenyl)-2-phenyl-5,6-dihydro-4H-1,3-thiazin-4-one (3d)}
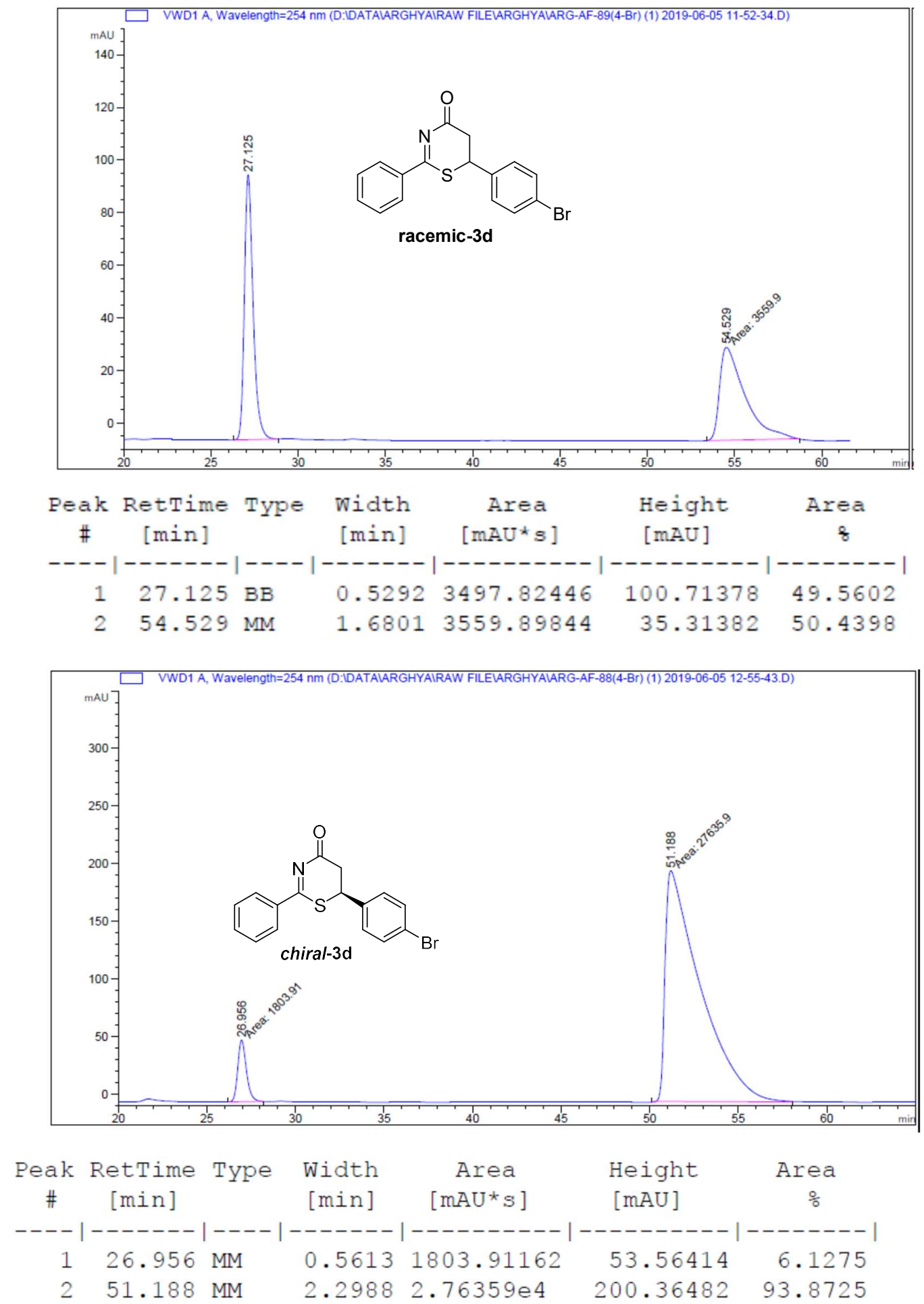

Sample Info : CHIRALPAK IF, 208 IPA-Hexane, $.7 \mathrm{~mL} / \mathrm{min}, 254 \mathrm{~nm}$ 
(S)-6-(4-Chlorophenyl)-2-phenyl-5,6-dihydro-4H-1,3-thiazin-4-one (3e)
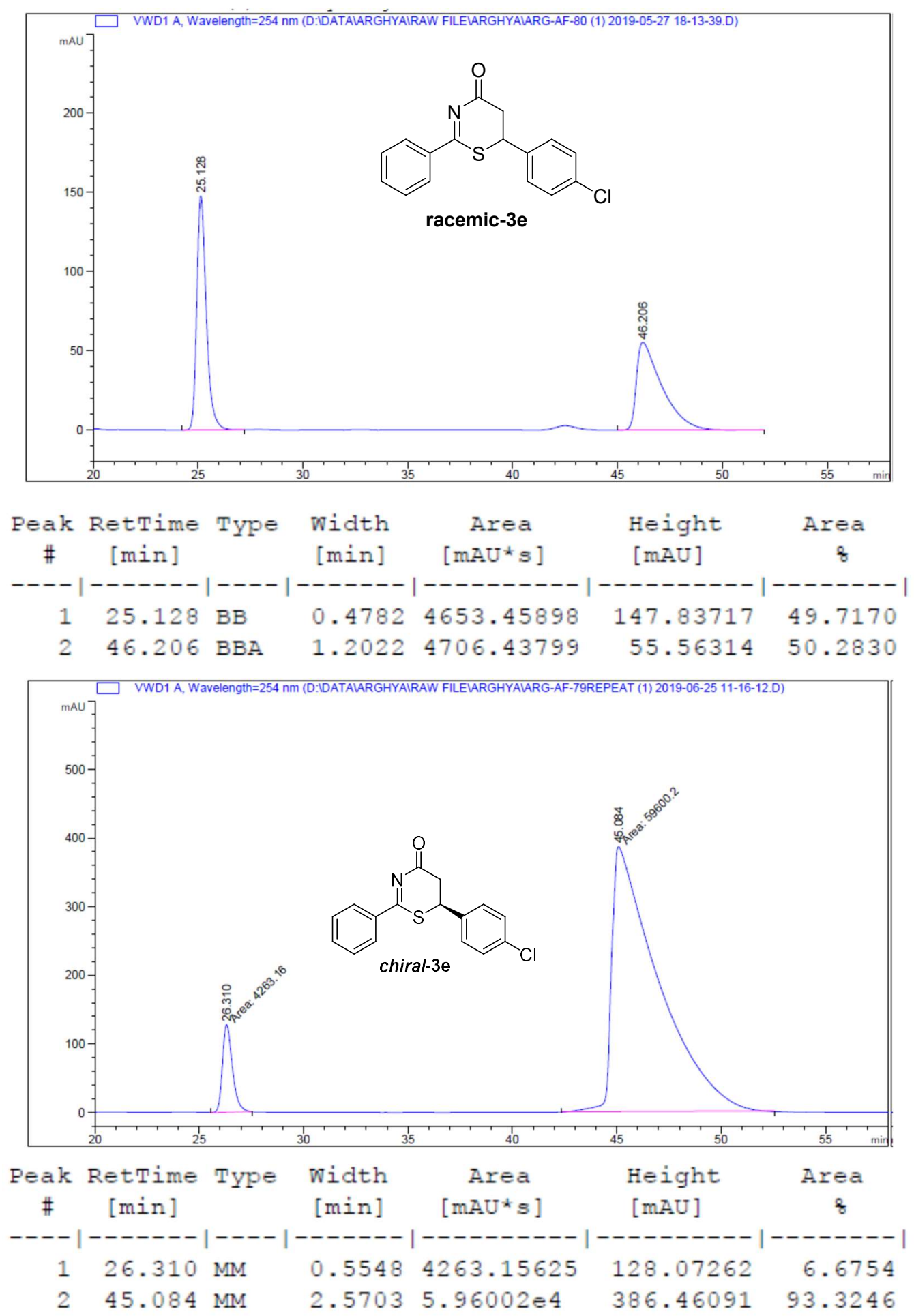

Sample Info : ChIRALPAR IF, 208IpA-Hexane, $.70 \mathrm{~mL} / \mathrm{min}, 254 \mathrm{~nm}$

S66 


\section{(S)-6-(4-Fluorophenyl)-2-phenyl-5,6-dihydro-4H-1,3-thiazin-4-one (3f)}
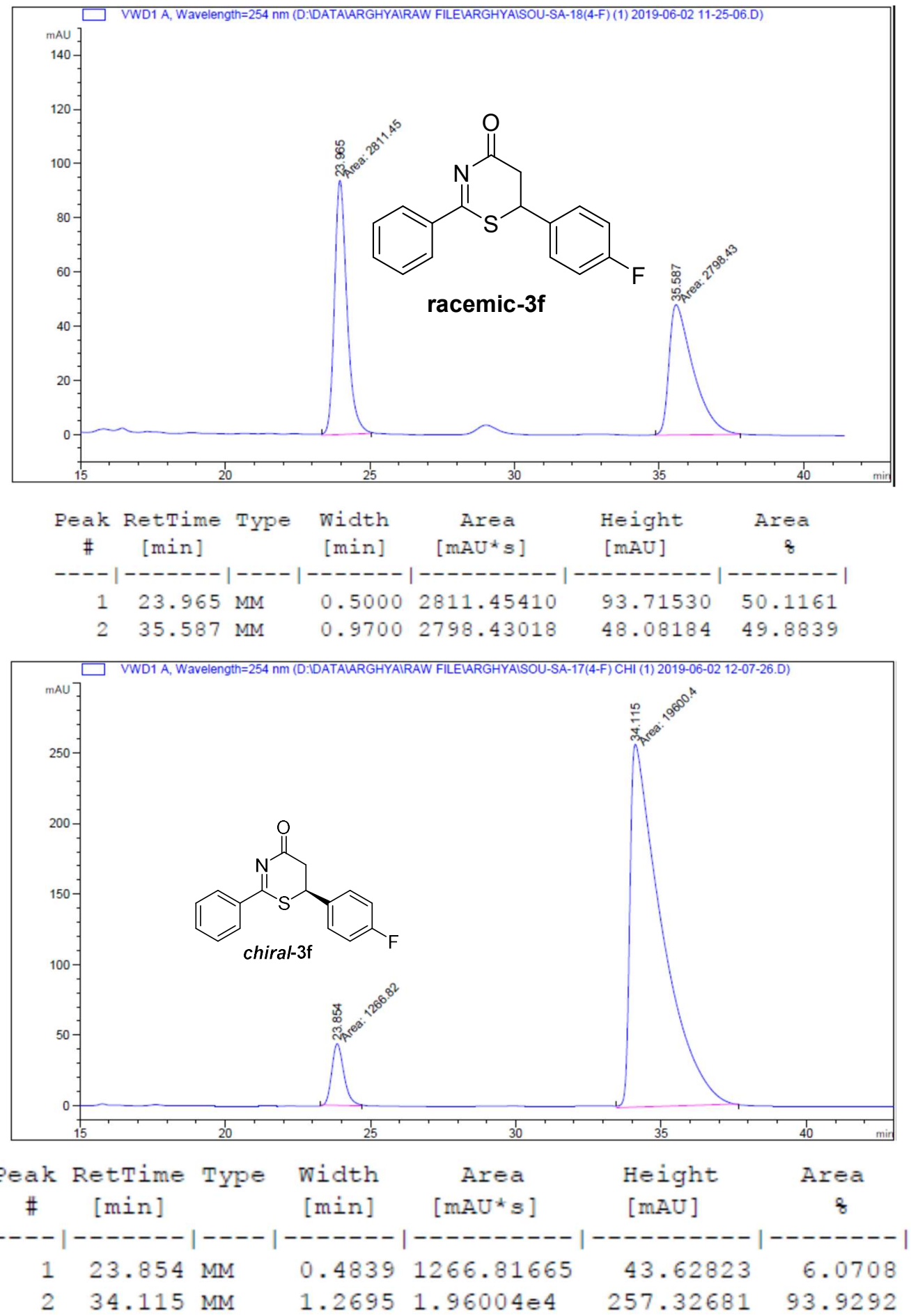

Sample Info

: CHIRALPAR IF, 208IPA-Hexane, .7 mL/min, $254 \mathrm{~nm}$ 
(S)-2-Phenyl-6-(4-(trifluoromethyl)phenyl)-5,6-dihydro-4H-1,3-thiazin-4-one (3g)
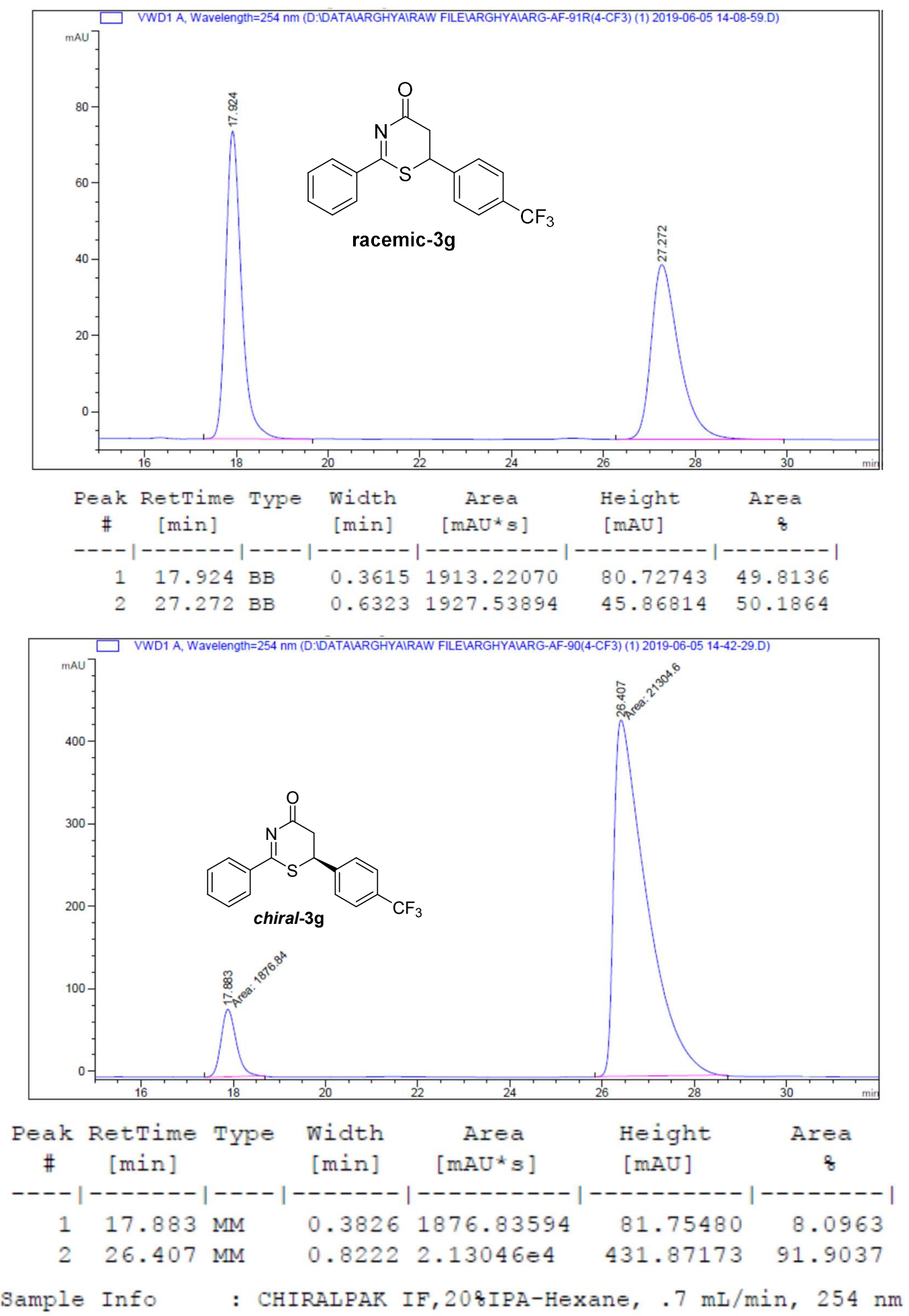
(S)-6-(4-Nitrophenyl)-2-phenyl-5,6-dihydro-4H-1,3-thiazin-4-one (3h)
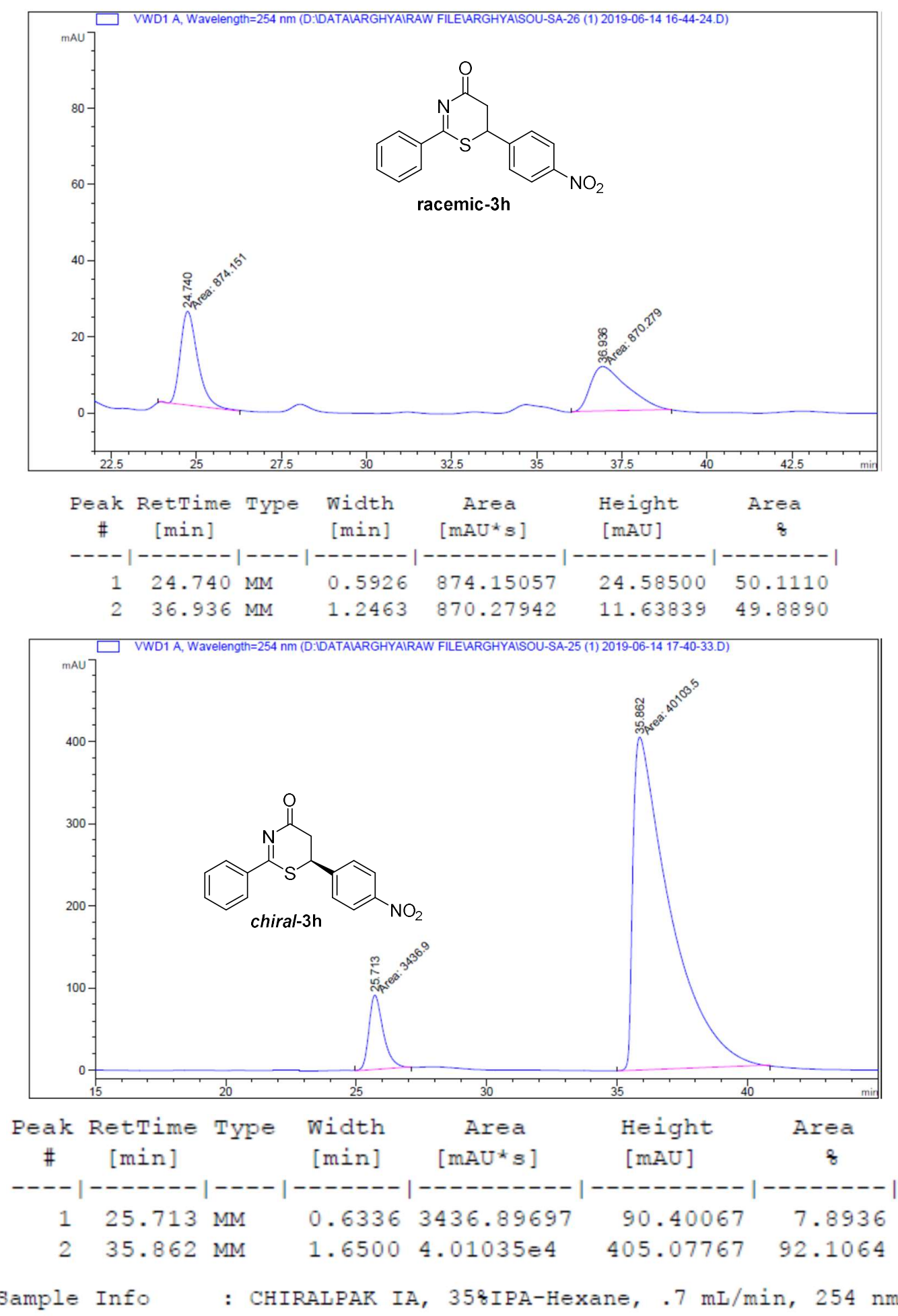


\section{( S)-6-(3-Bromophenyl)-2-phenyl-5,6-dihydro-4H-1,3-thiazin-4-one (3i)}
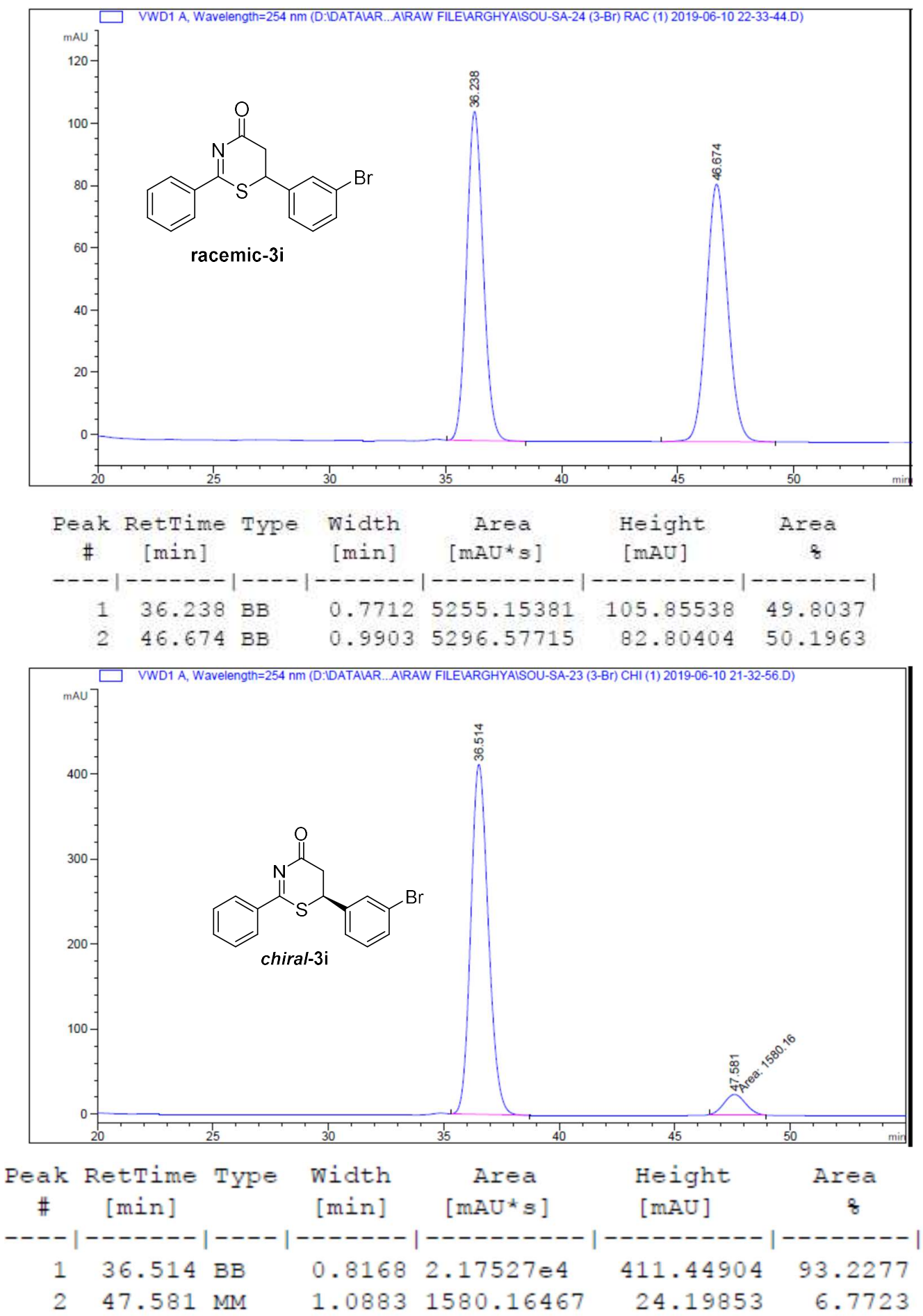

Sample Info : CHIRALPAK IC, 308 IPA-Hexane, $.7 \mathrm{~mL} / \mathrm{min}, 254 \mathrm{~nm}$ 


\section{( S)-6-(3-Chlorophenyl)-2-phenyl-5,6-dihydro-4H-1,3-thiazin-4-one (3j)}
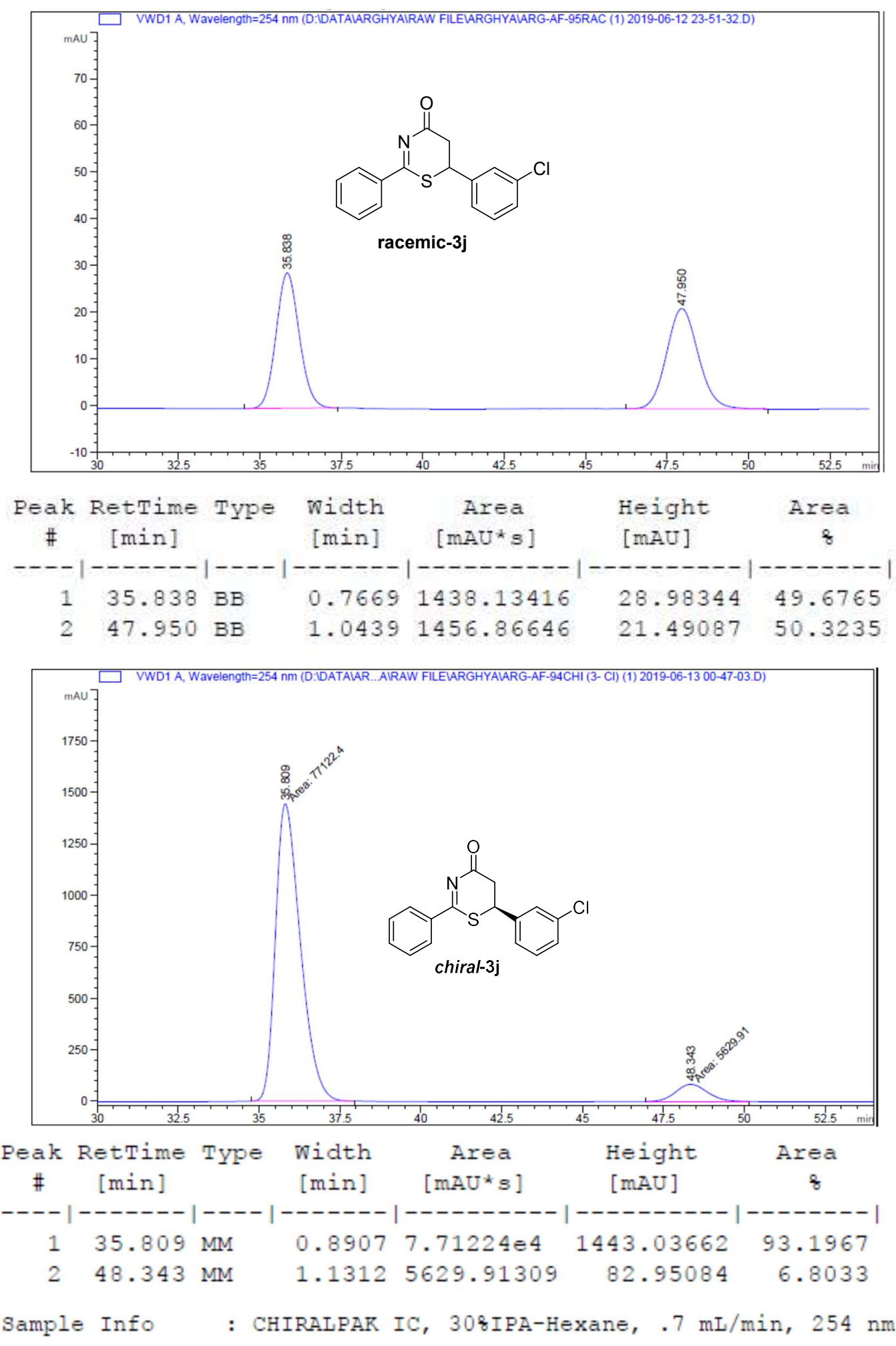
(S)-6-(3-Nitrophenyl)-2-phenyl-5,6-dihydro-4H-1,3-thiazin-4-one (3k)
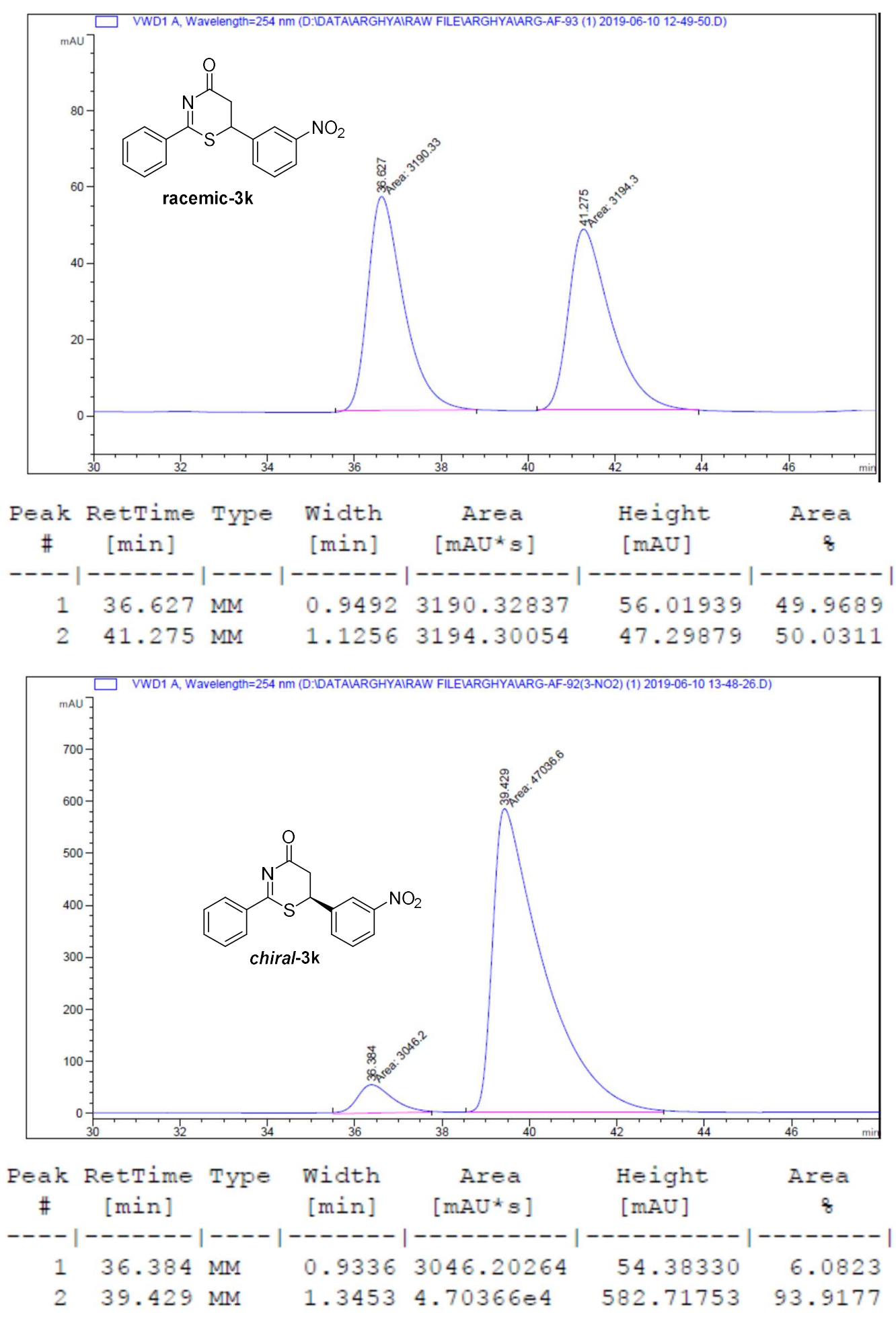

Sample Info : CHIRALPAR IF, 308 IPA-Hexane, $.7 \mathrm{~mL} / \mathrm{min}, 254 \mathrm{~nm}$ 


\section{(S)-6-(2-Methoxyphenyl)-2-phenyl-5,6-dihydro-4H-1,3-thiazin-4-one (3I)}
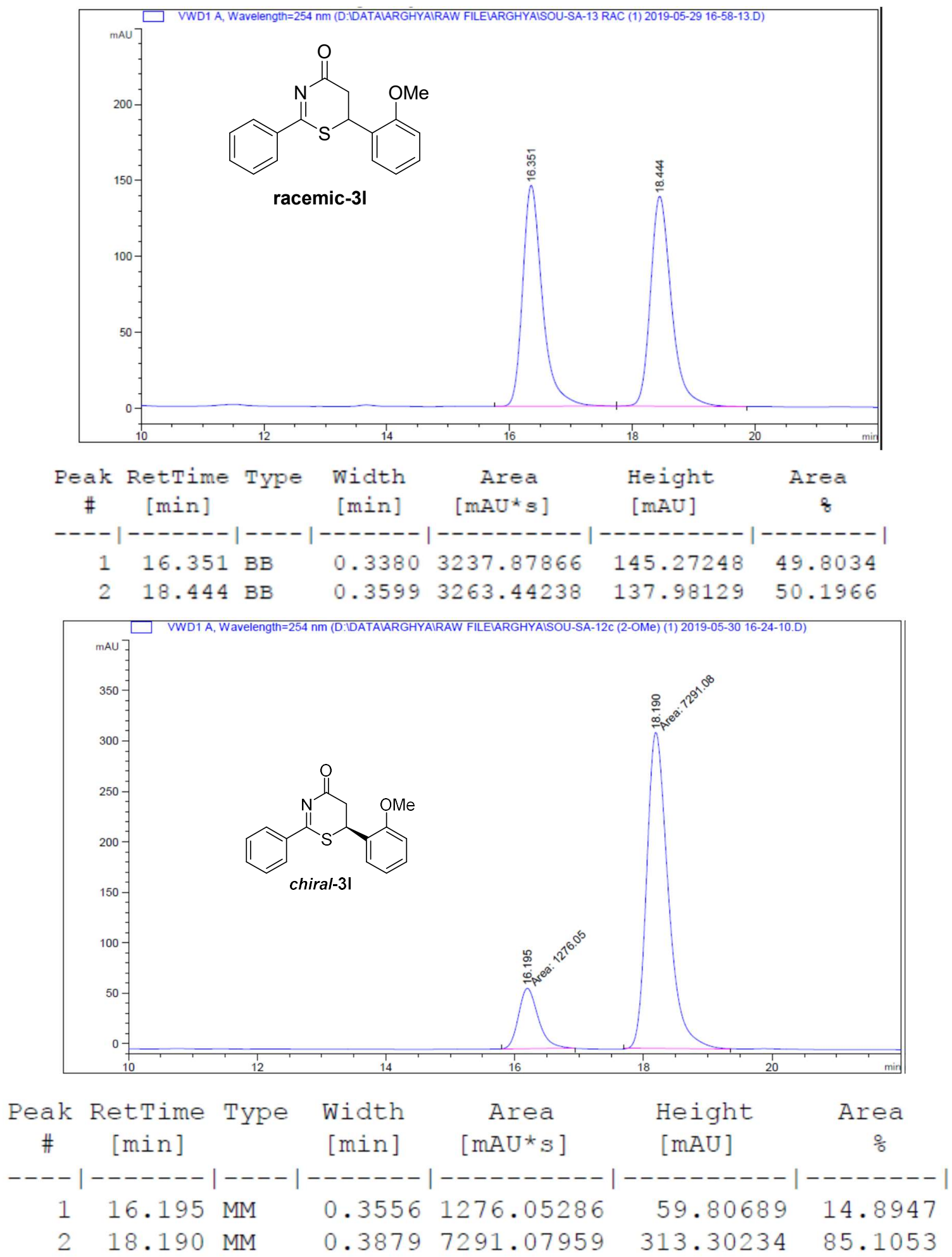

Sample Info: CHIRALPAK IF, 30IPA-Hexane, $.7 \mathrm{~mL} / \mathrm{min}, 254 \mathrm{~nm}$ 


\section{(S)-6-(2-Bromophenyl)-2-phenyl-5,6-dihydro-4H-1,3-thiazin-4-one (3m)}
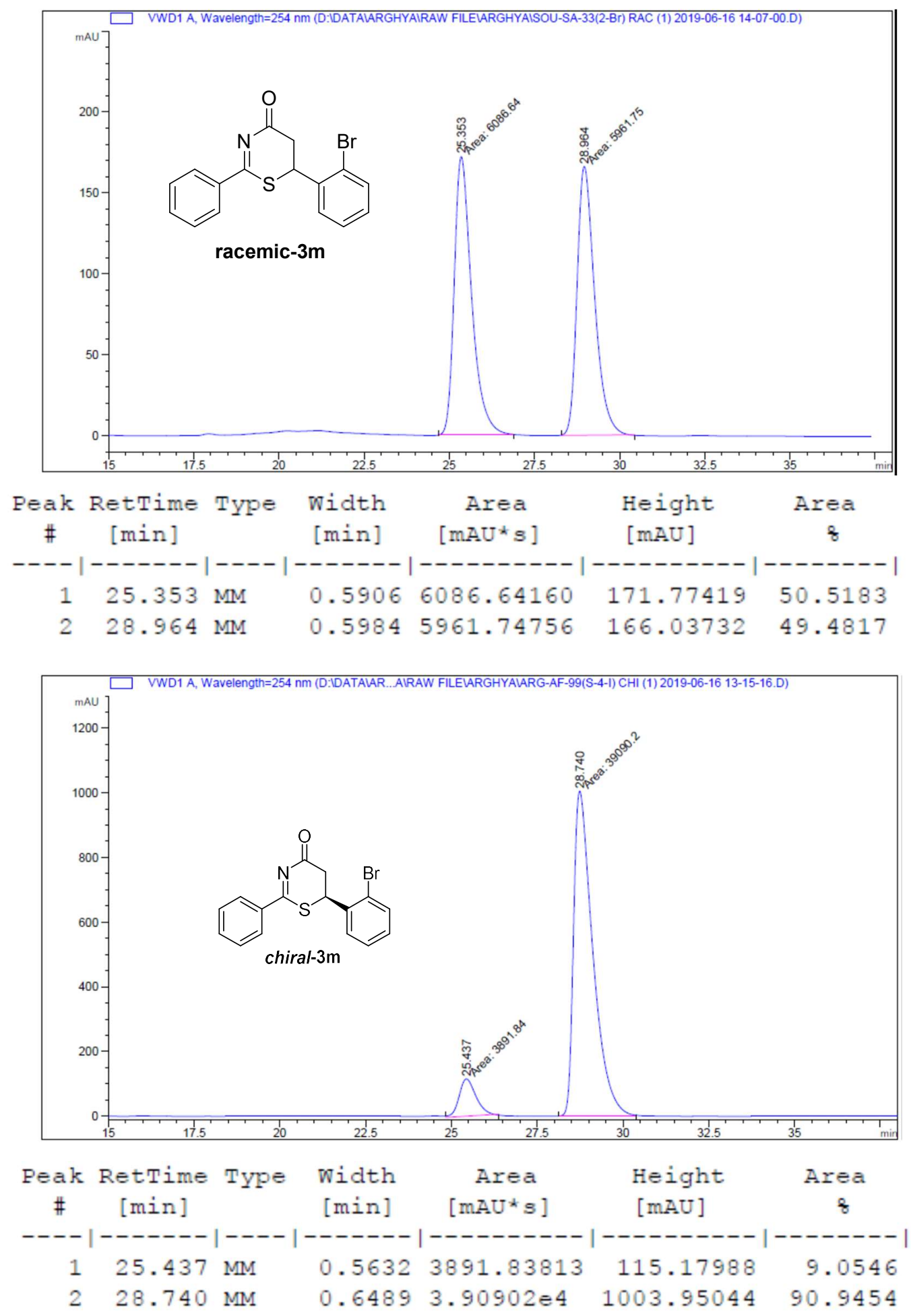

Sample Info: CHIRALPAK IF, $208 I P A-H e x a n e, .7 \mathrm{~mL} / \mathrm{min}, 254 \mathrm{~nm}$ 


\section{(S)-6-(2-Chlorophenyl)-2-phenyl-5,6-dihydro-4H-1,3-thiazin-4-one (3n)}
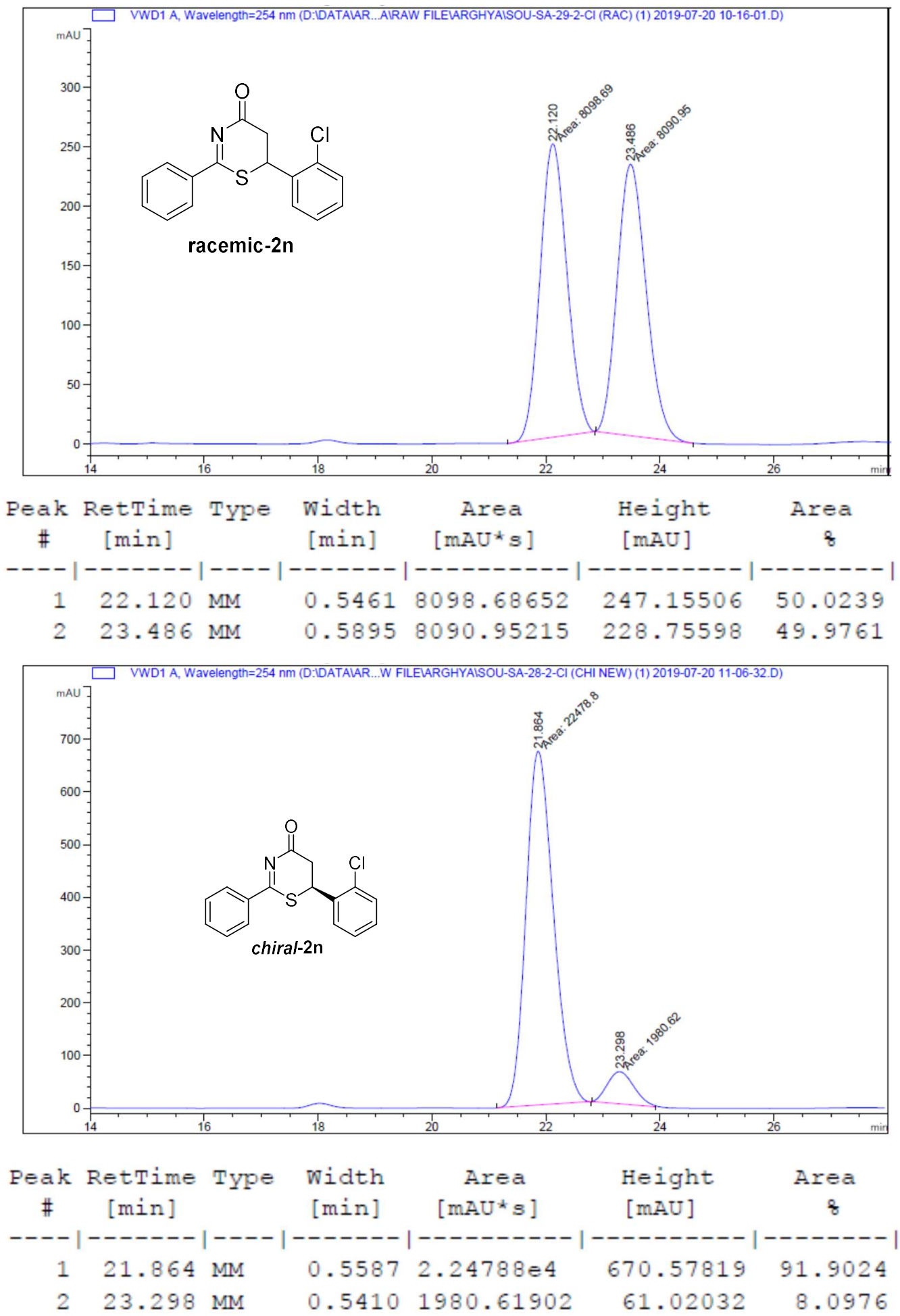

Sample Info : CHIRALPAK IC, 308 IPA-Hexane, $1.0 \mathrm{~mL} / \mathrm{min}, 254 \mathrm{~nm}$ 
(S)-6-(3,4-Dichlorophenyl)-2-phenyl-5,6-dihydro-4H-1,3-thiazin-4-one (3o)
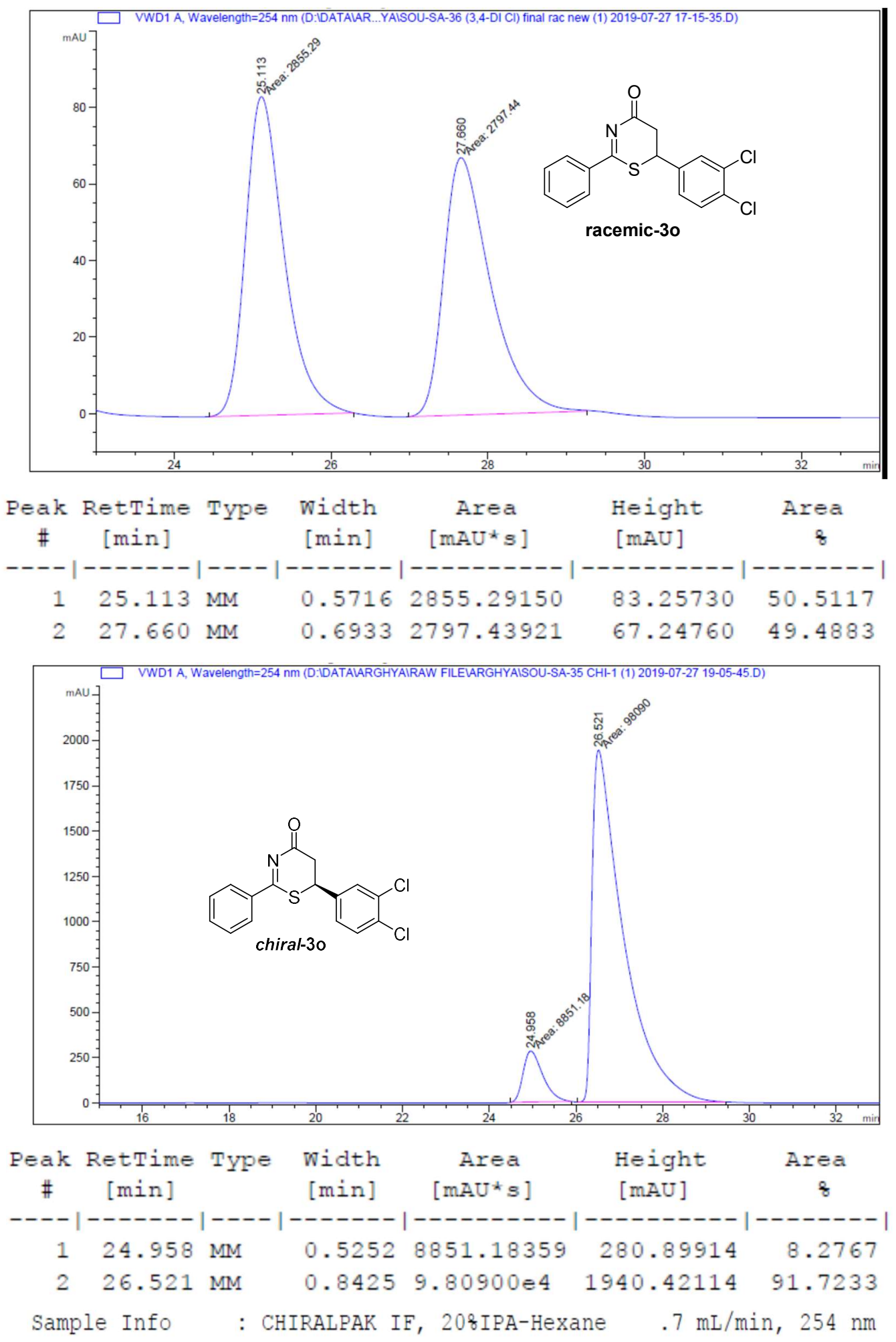
(S)-6-(3-Bromo-4-methoxyphenyl)-2-phenyl-5,6-dihydro-4H-1,3-thiazin-4-one (3p)
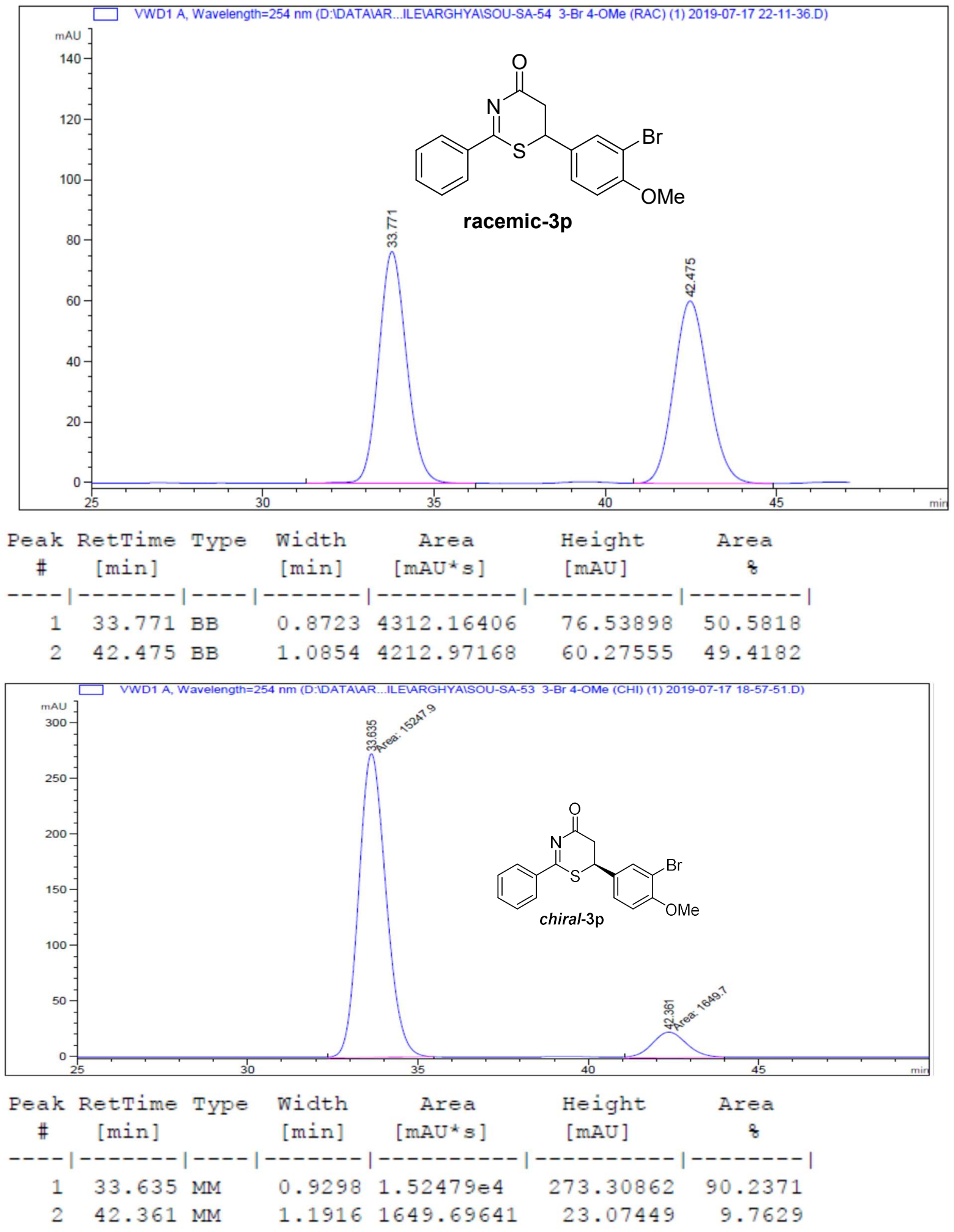

Sample Info

: CHIRALPAR IC, 308 IPA-Hexane, $1.0 \mathrm{~mL} / \mathrm{min}, 254 \mathrm{~nm}$ 
(S)-6-(2-Bromo-4,5-dimethoxyphenyl)-2-phenyl-5,6-dihydro-4H-1,3-thiazin-4-one (3q)
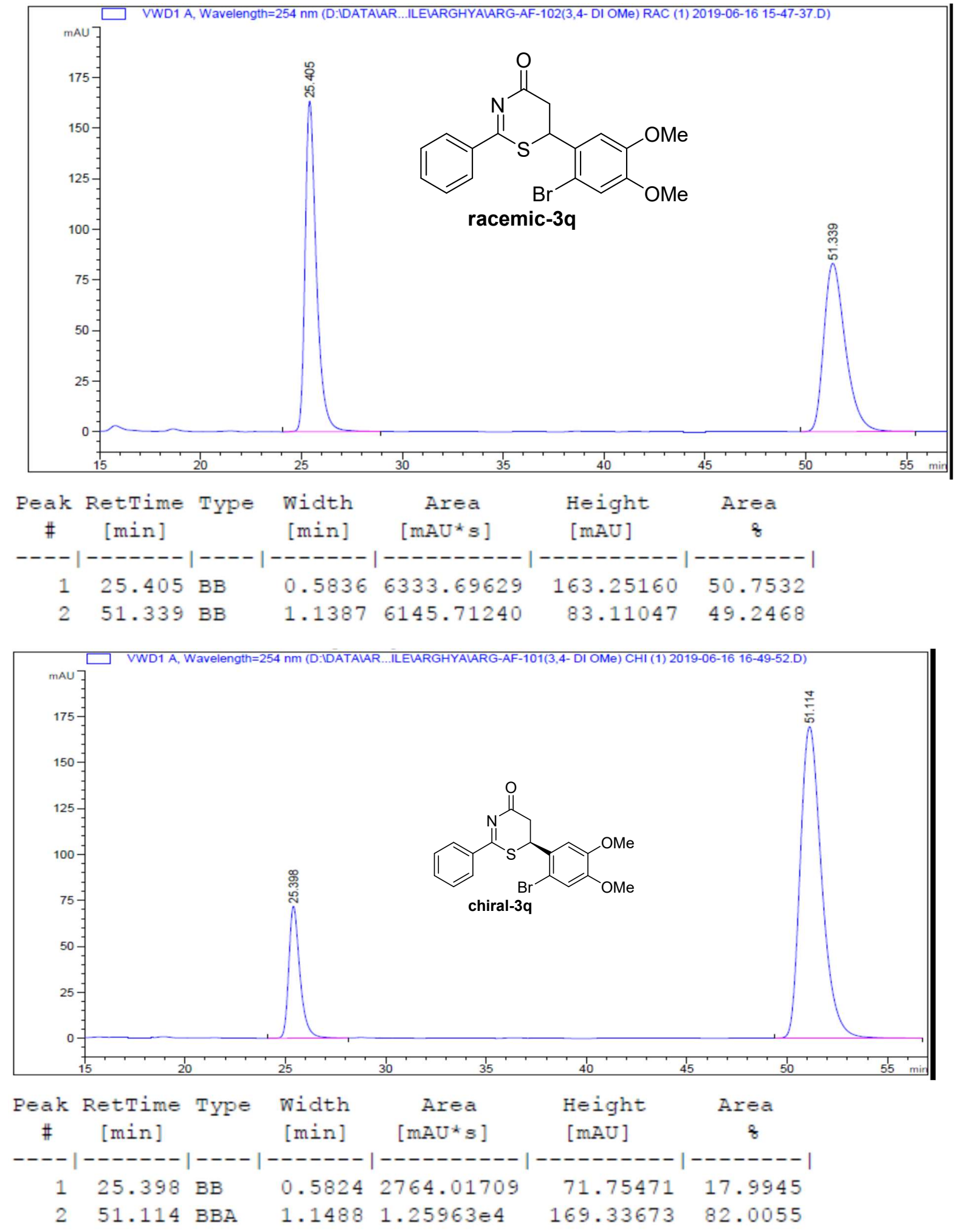

Sample Info : CHIRALPAK IF, 308 IPA-Hexane, .7 mL/min, $254 \mathrm{~nm}$ 
(S)-6-(Furan-2-yl)-2-phenyl-5,6-dihydro-4H-1,3-thiazin-4-one (3r)
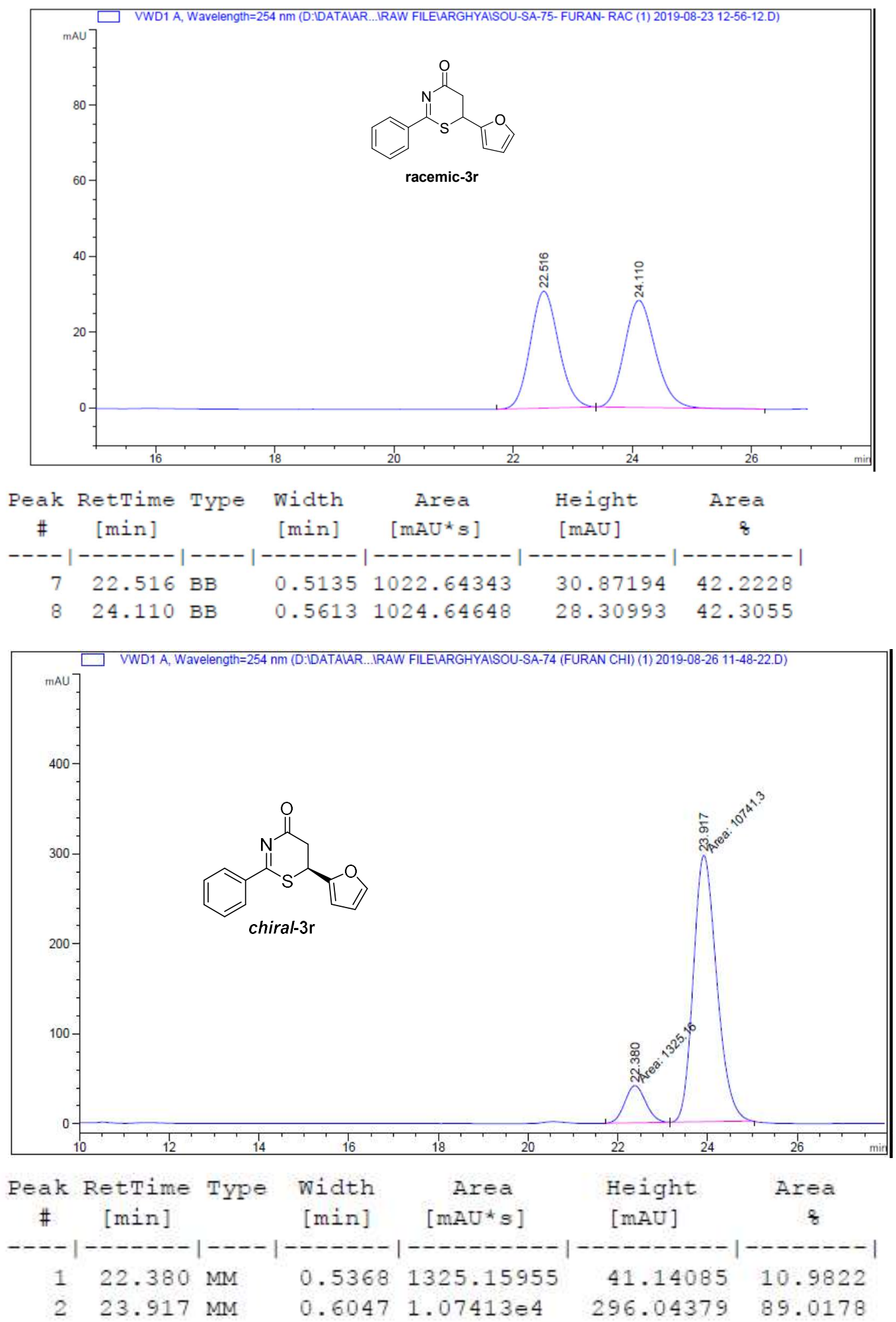

Sample Info : CHIRALPAK IC, 308 IPA-HEXANE, $1 \mathrm{~mL}-\mathrm{min}, 254 \mathrm{~nm}$ 


\section{(S)-2-Phenyl-6-(thiophen-2-yl)-5,6-dihydro-4H-1,3-thiazin-4-one (3s)}
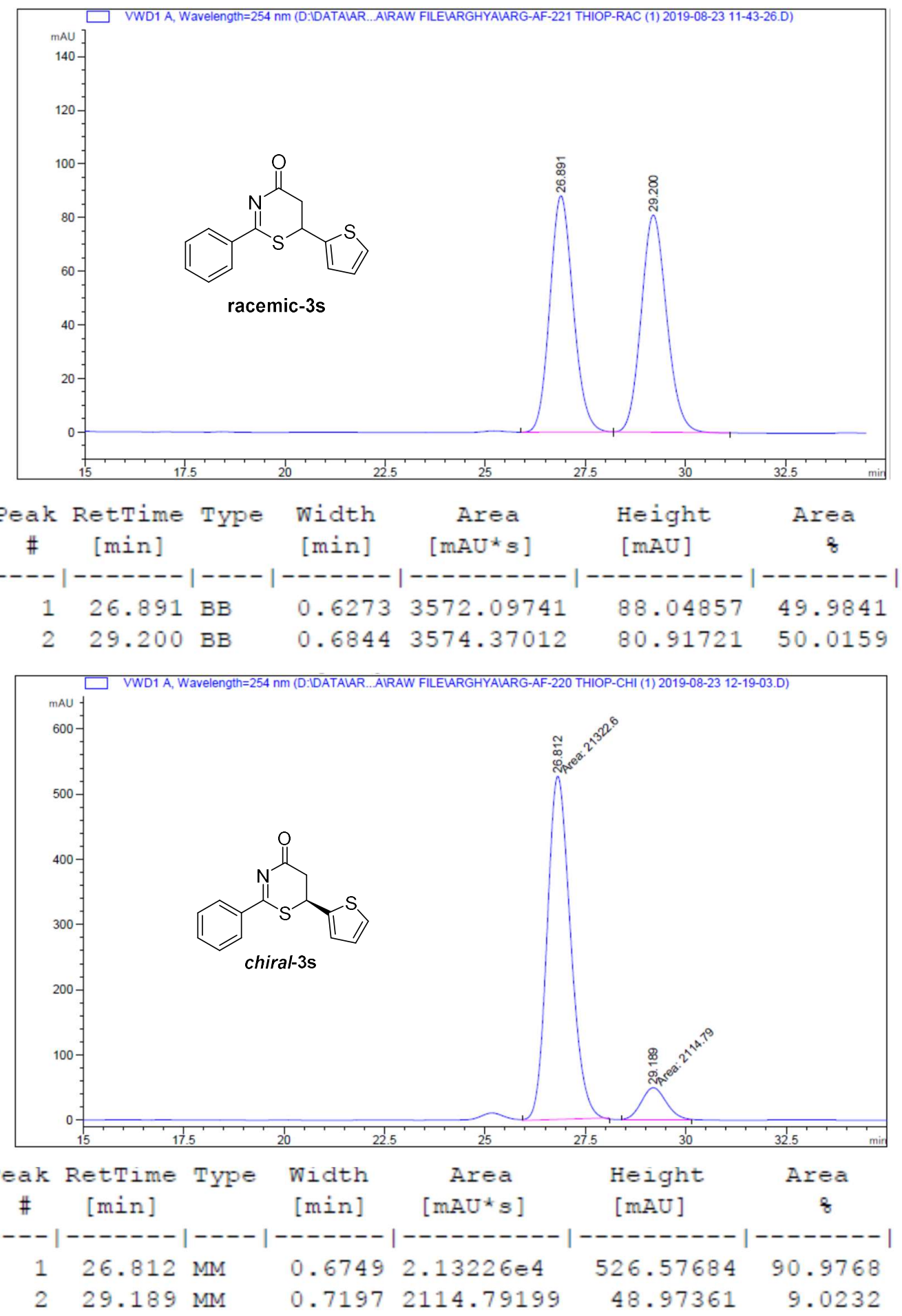


\section{(R)-6-Methyl-2-phenyl-5,6-dihydro-4H-1,3-thiazin-4-one (3t)}
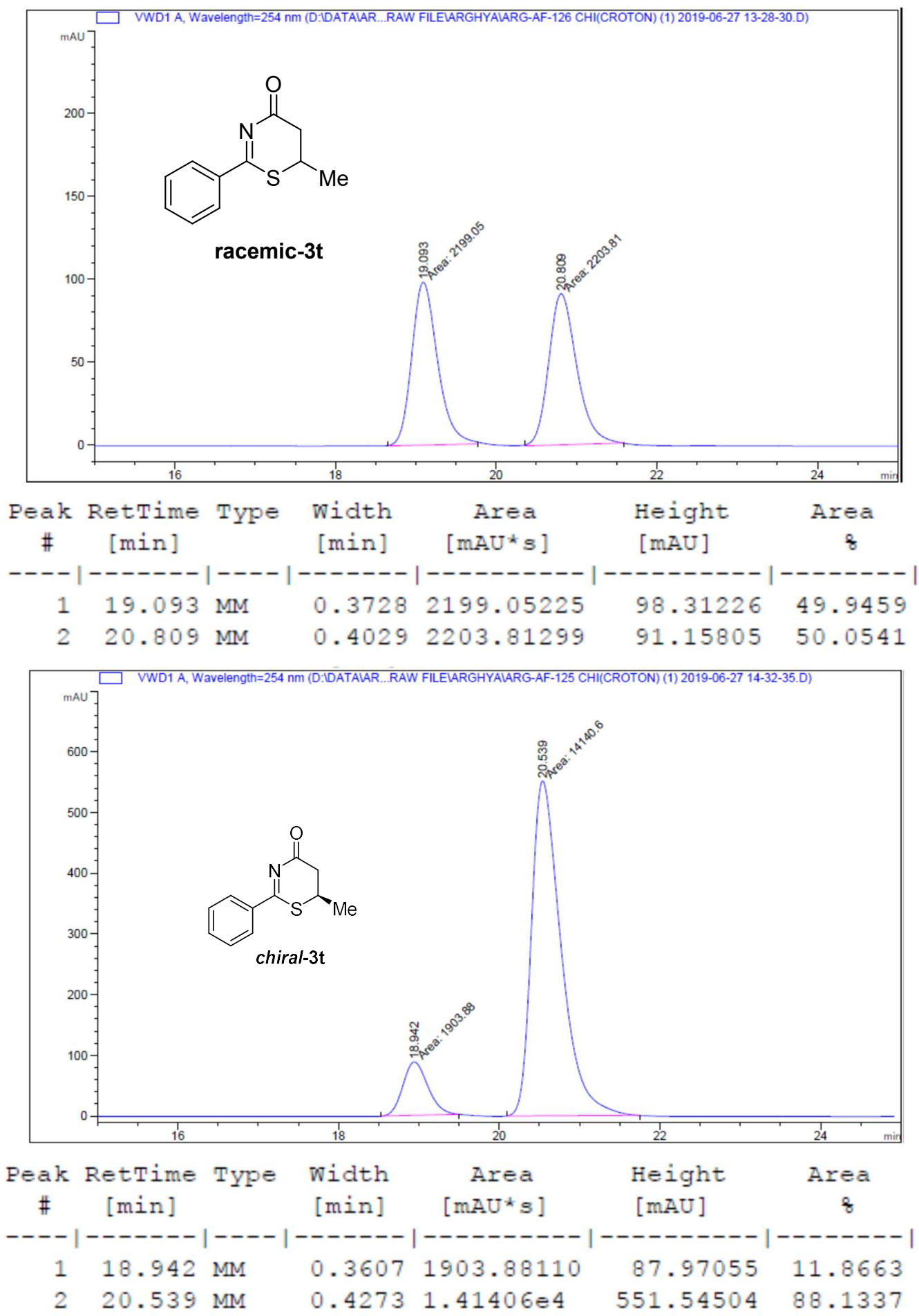

Sample Info

: CHIRALPAK IF, 208 IPA-Hexane, . $7 \mathrm{~mL} / \mathrm{min}, 254 \mathrm{~nm}$ 


\section{(R)-6-Ethyl-2-phenyl-5,6-dihydro-4H-1,3-thiazin-4-one(3u)}
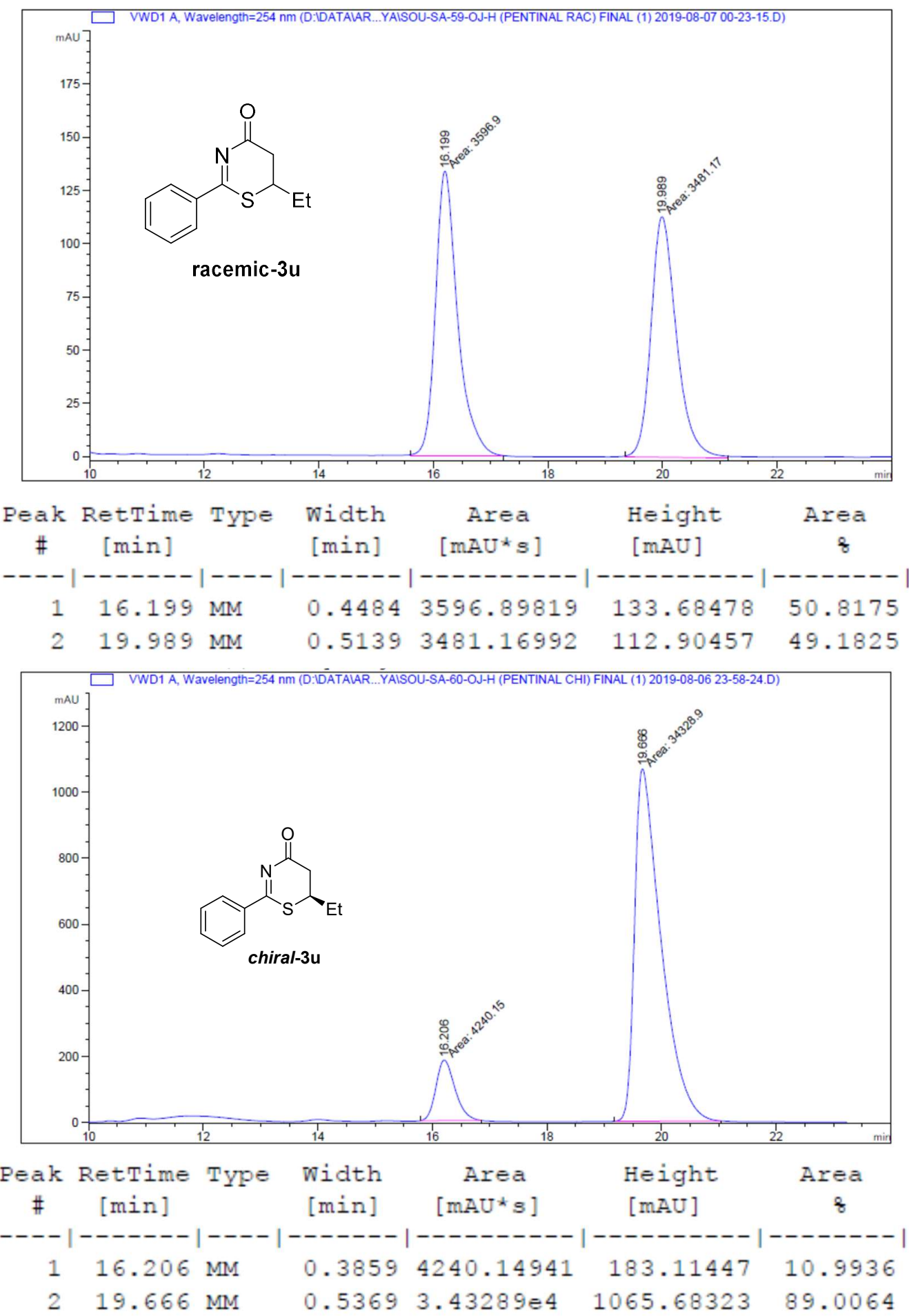

Sample Info

: CHIRALCEL

OJ-H, 158 IPA-HEXANE, $1 \mathrm{~mL}-\mathrm{min}$,

$254 \mathrm{~nm}$ 


\section{(S)-2-(4-Methoxyphenyl)-6-phenyl-5,6-dihydro-4H-1,3-thiazin-4-one (3v)}
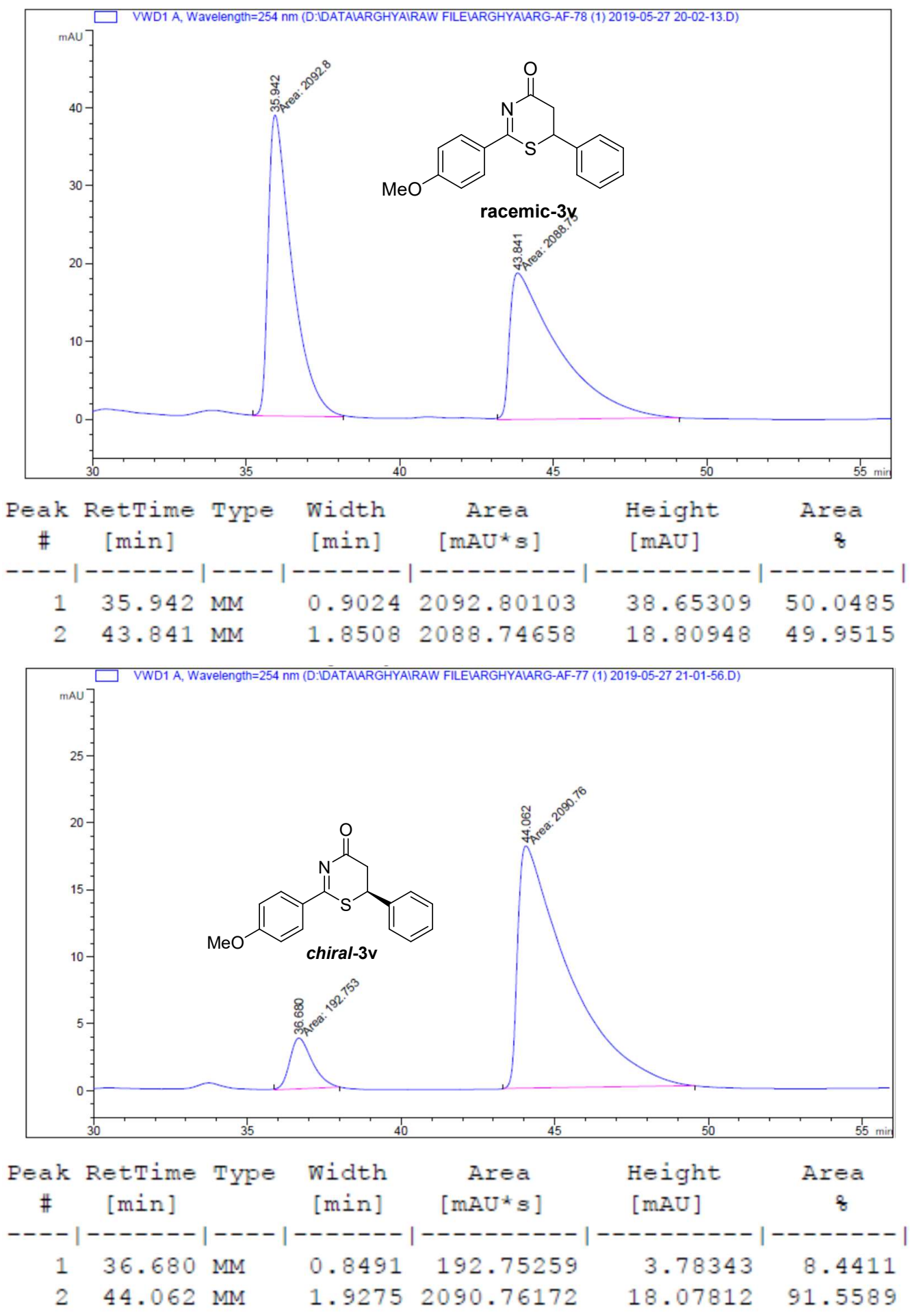

Sample Info : CHIRALPAR IF, 258 IPA-Hexane, $.7 \mathrm{~mL} / \mathrm{min}, 254 \mathrm{~nm}$ 


\section{(S)-6-Phenyl-2-(p-tolyl)-5,6-dihydro-4H-1,3-thiazin-4-one (3w)}
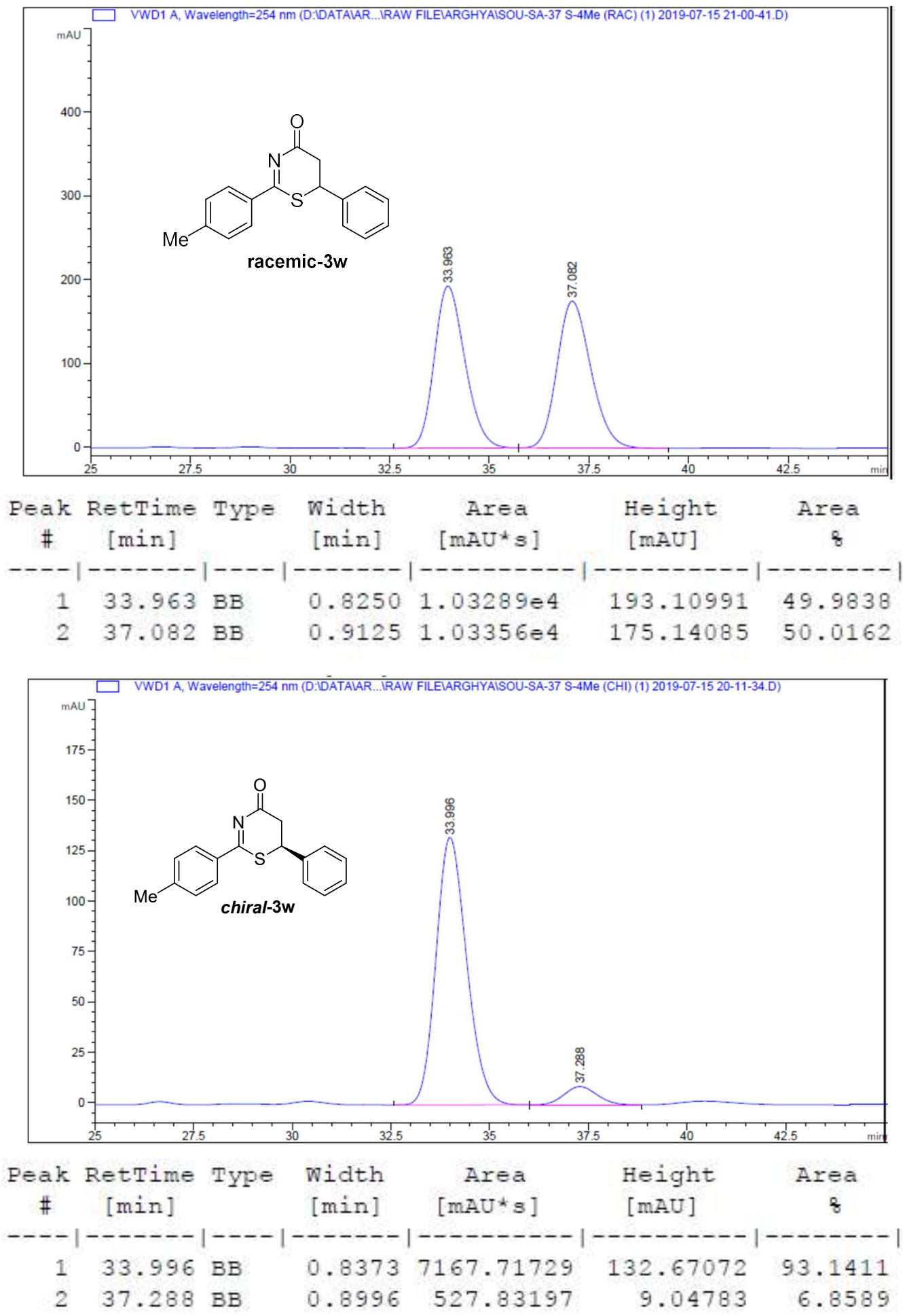

Sample Info : CHIRALPAK IC, 308 IPA-Hexane, $1.0 \mathrm{~mL} / \mathrm{min}, 254 \mathrm{~nm}$ 


\section{(S)-2-(4-Iodophenyl)-6-phenyl-5,6-dihydro-4H-1,3-thiazin-4-one (3x)}
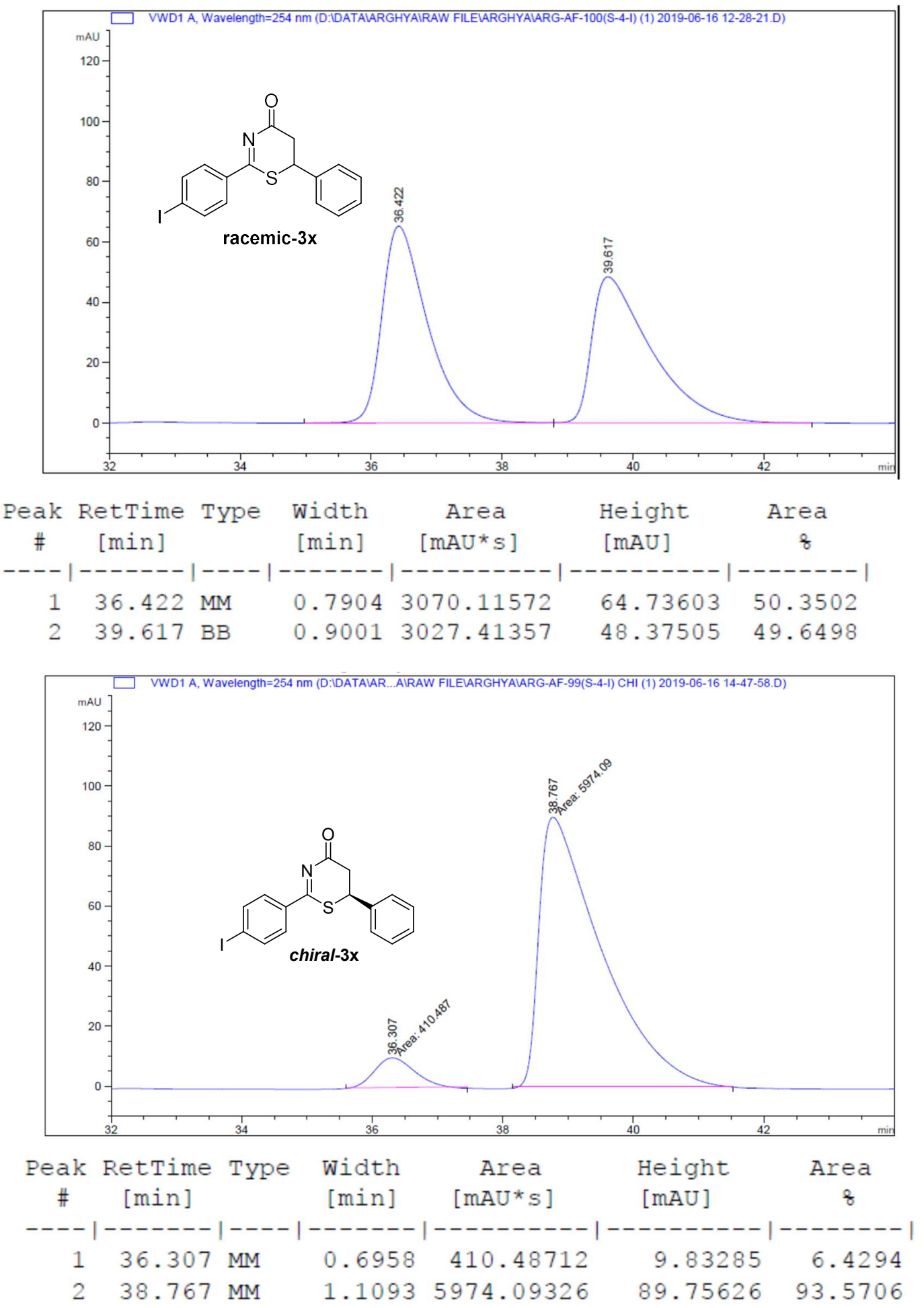

Sample Info : CHIRALPAK IF, 208 IPA-Hexane, $.7 \mathrm{~mL} / \mathrm{min}, 254 \mathrm{~nm}$ 


\section{(S)-2-(4-Bromophenyl)-6-phenyl-5,6-dihydro-4H-1,3-thiazin-4-one (3y)}
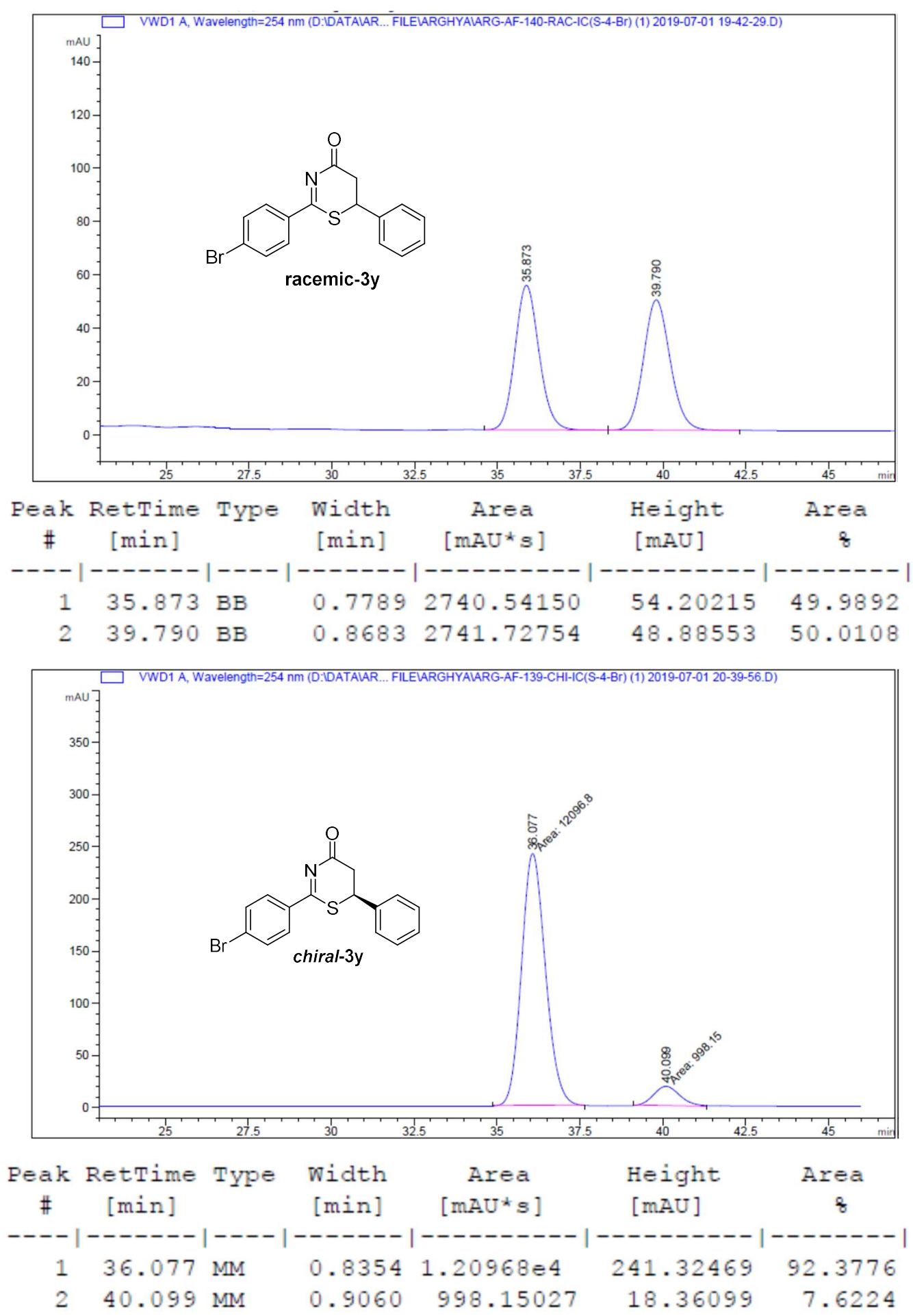

Sample Info : CHIRALPAK IC, 308 IPA-Hexane, $.7 \mathrm{~mL} / \mathrm{min}, 254 \mathrm{~nm}$ 


\section{(S)-2-(3-Methoxyphenyl)-6-phenyl-5,6-dihydro-4H-1,3-thiazin-4-one (3z)}
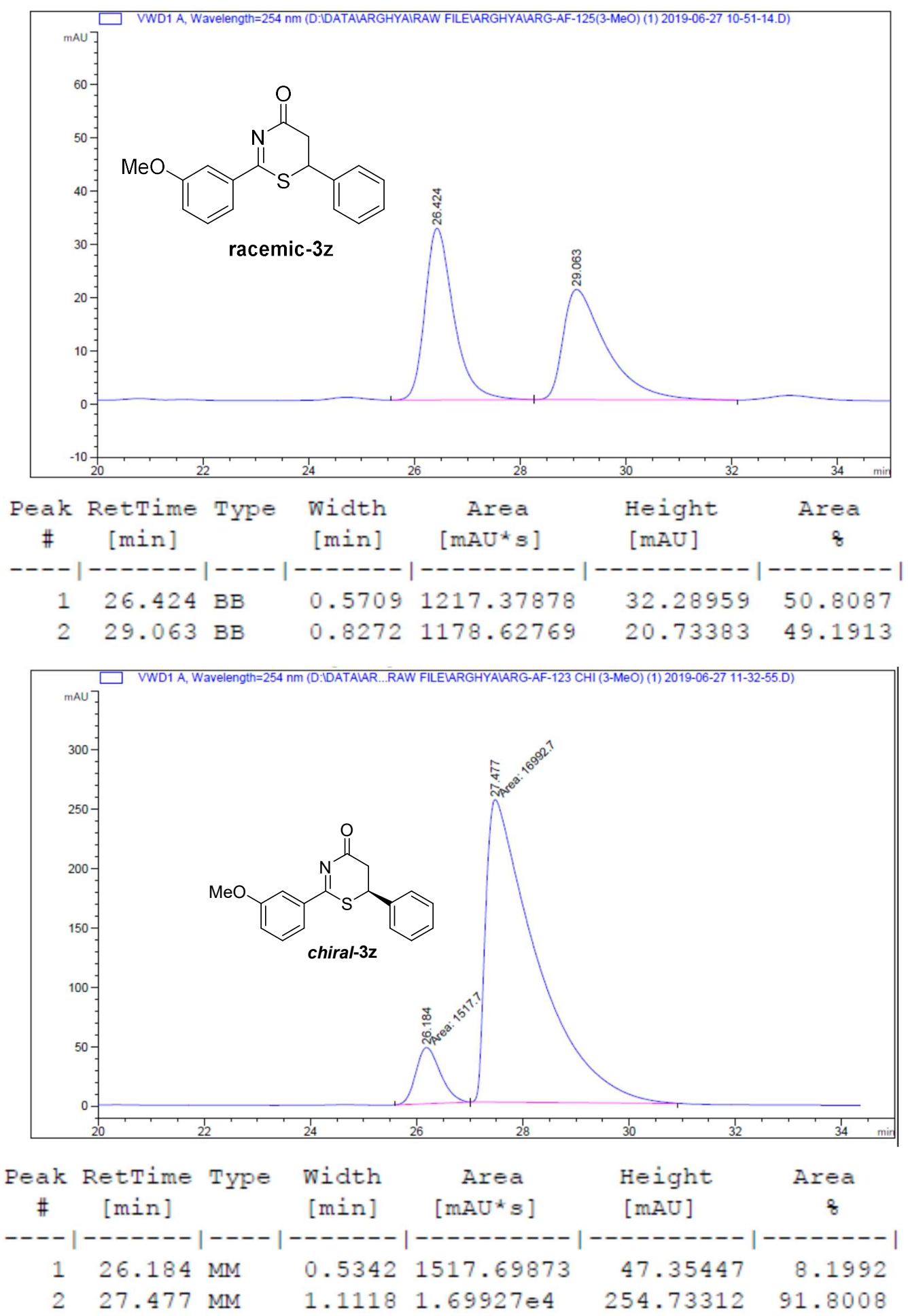

Sample Info: CHIRALPAK IF, 308 IPA-Hexane, $.7 \mathrm{~mL} / \mathrm{min}, 254 \mathrm{~nm}$ 


\section{(S)-6-Phenyl-2-(m-tolyl)-5,6-dihydro-4H-1,3-thiazin-4-one (3aa)}

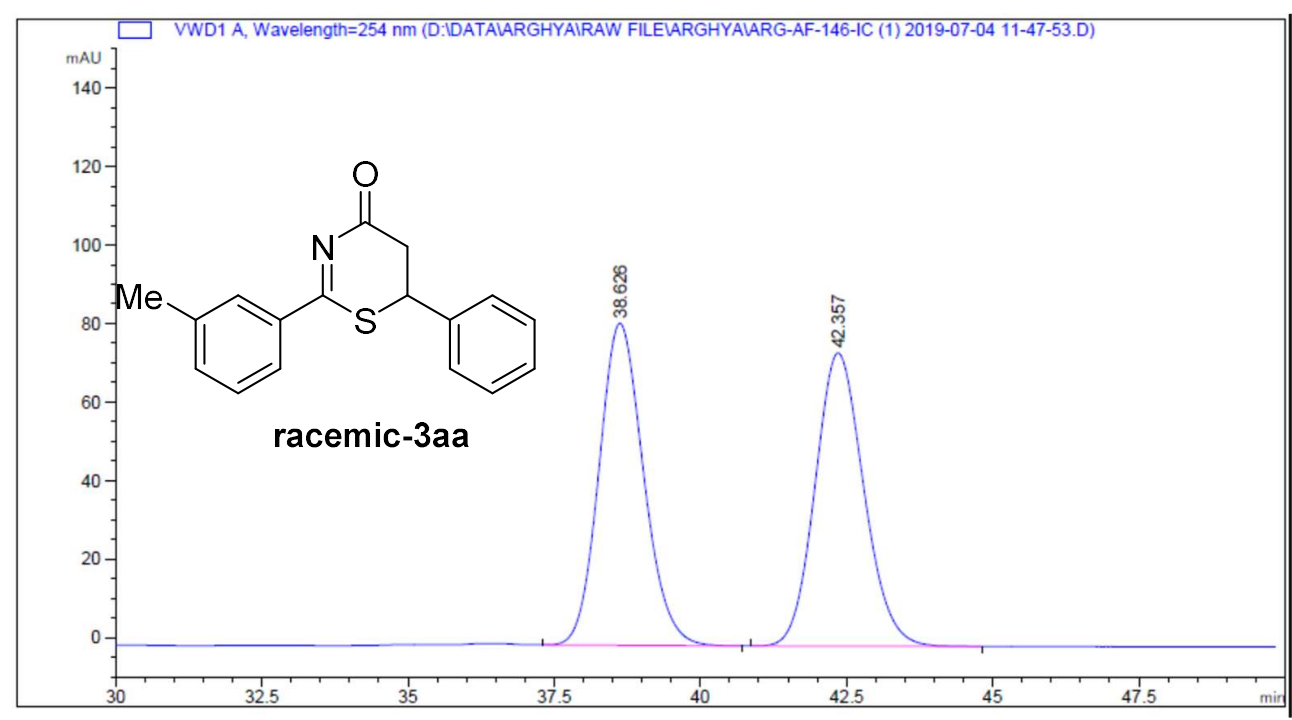

\begin{tabular}{|c|c|c|c|c|c|c|}
\hline $\begin{array}{c}\text { Peak } \\
\#\end{array}$ & $\begin{array}{c}\text { RetTime } \\
\text { [min] }\end{array}$ & Type & $\begin{array}{c}\text { Width } \\
\text { [min] }\end{array}$ & $\begin{array}{c}\text { Area } \\
{\left[\mathrm{mAU}^{*} \mathrm{~s}\right]}\end{array}$ & $\begin{array}{l}\text { Height } \\
{[\mathrm{mAU}]}\end{array}$ & $\begin{array}{c}\text { Area } \\
\&\end{array}$ \\
\hline & ---0 & - & --- & ---------- & ---- & ---- \\
\hline 1 & 38.626 & MM & 0.8771 & 4310.63379 & 81.91376 & 49.8543 \\
\hline 2 & 42.357 & MM & 0.9663 & 4335.83789 & 74.78542 & 50.1457 \\
\hline
\end{tabular}

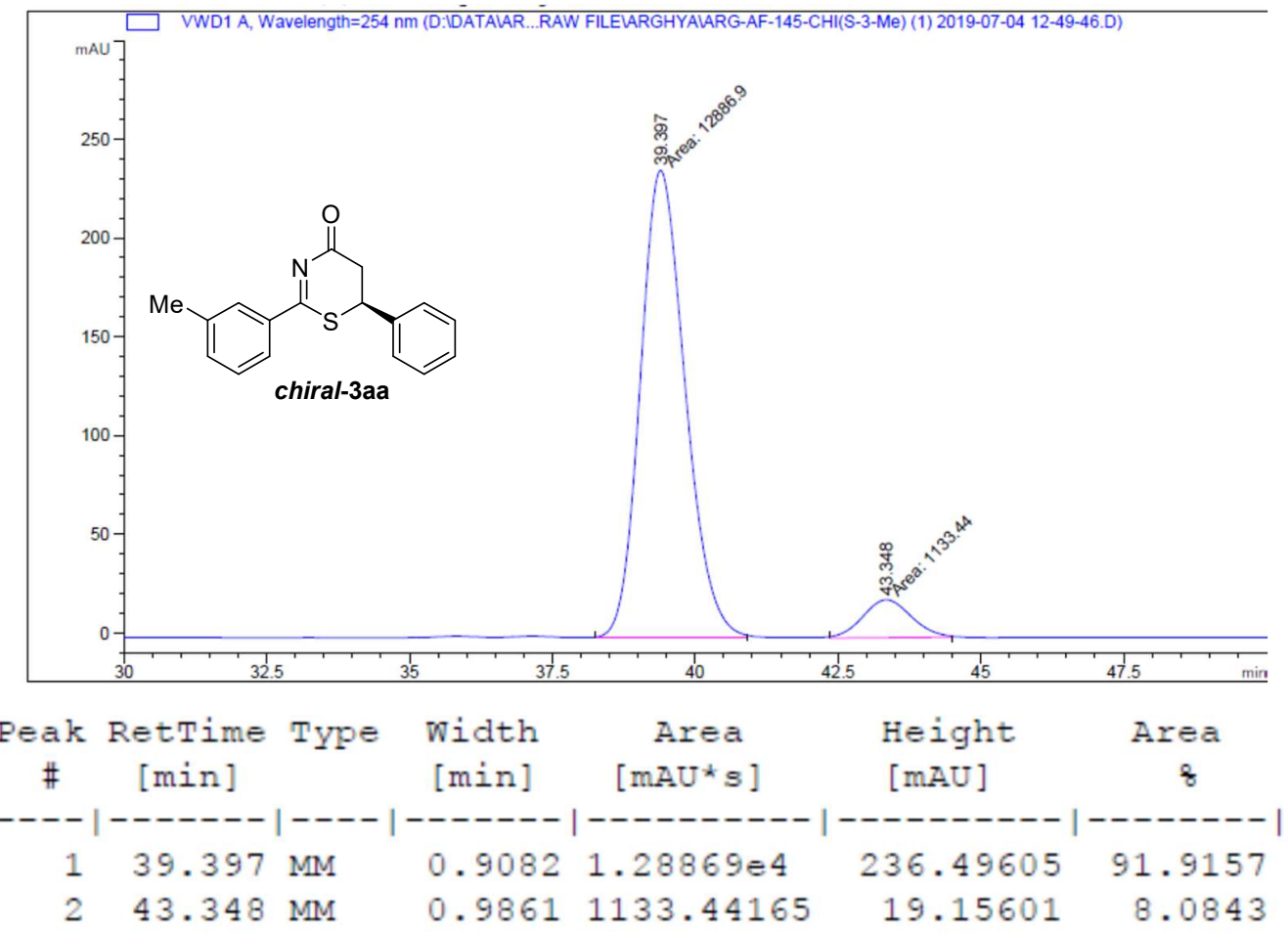

Sample Info

: CHIRALPAR IC, 308 IPA-Hexane, $.7 \mathrm{~mL} / \mathrm{min}, 254 \mathrm{~nm}$ 


\section{(S)-2-(3-Bromophenyl)-6-phenyl-5,6-dihydro-4H-1,3-thiazin-4-one (3ab)}
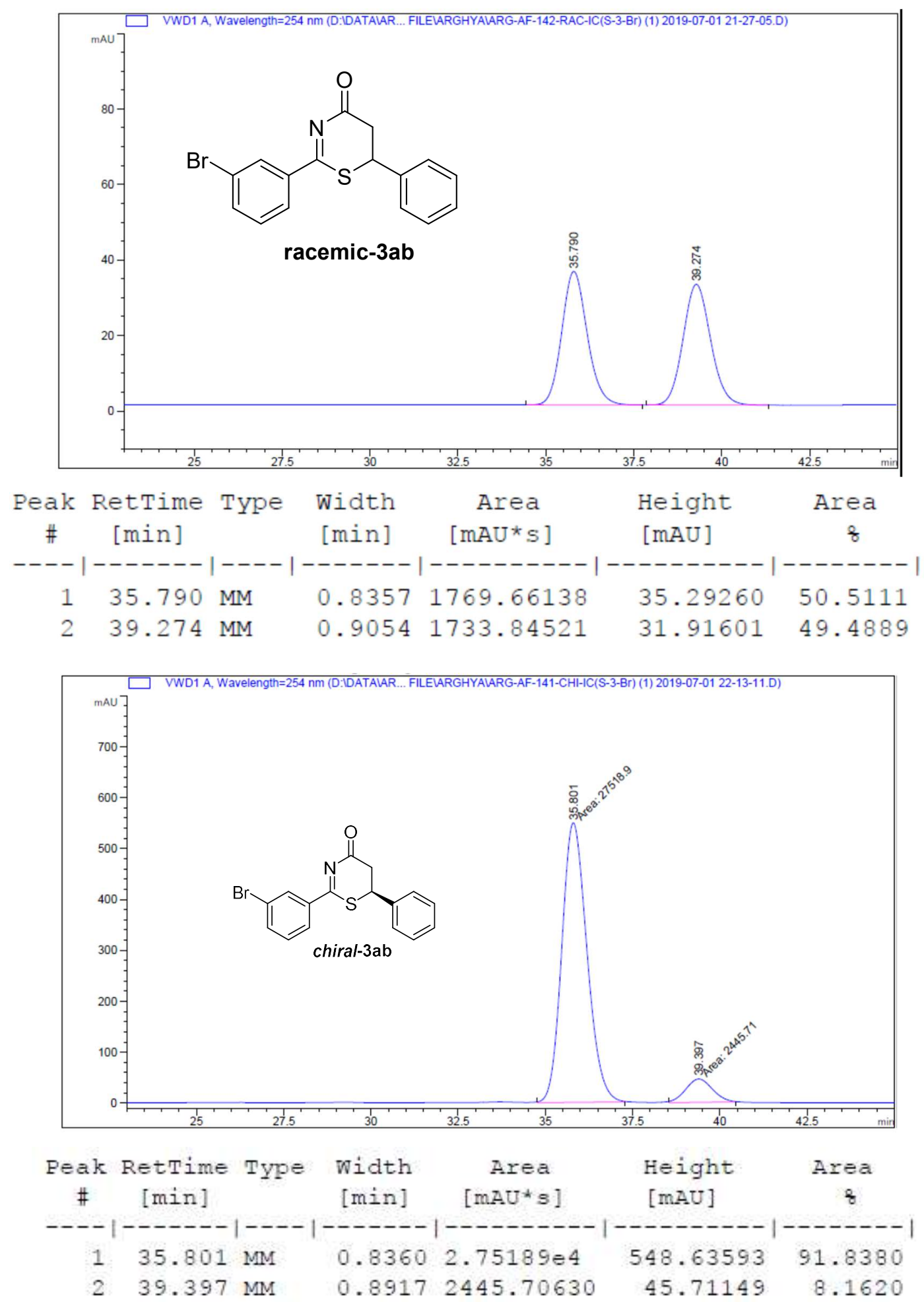

Sample Info

: CHIRAIPAK IC, $308 I P A-H e x a n e, .7 \mathrm{~mL} / \mathrm{min}, 254 \mathrm{~nm}$ 


\section{(S)-2-(3-Nitrophenyl)-6-phenyl-5,6-dihydro-4H-1,3-thiazin-4-one (3ac)}
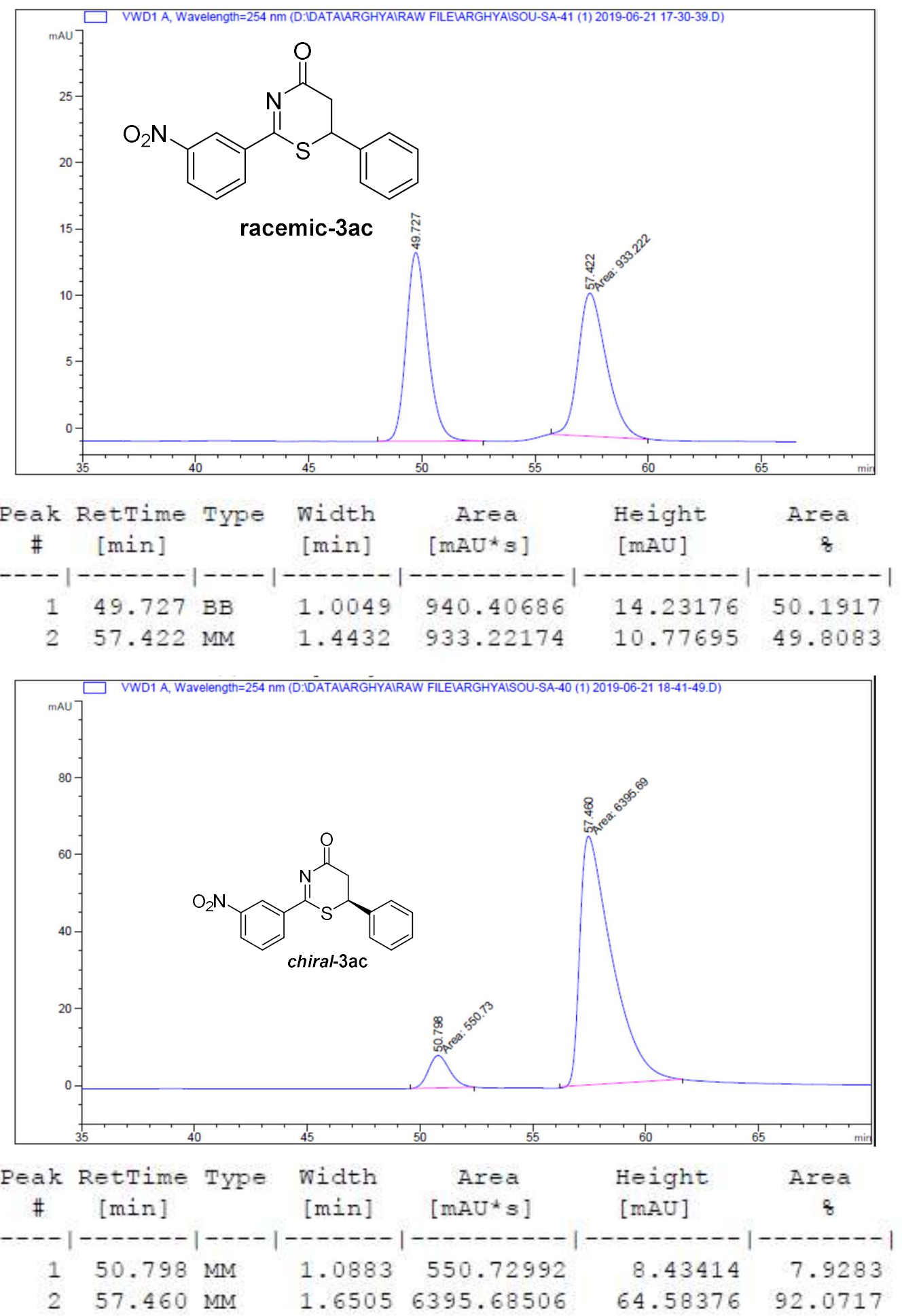

Sample Info : ChIRALPAK IF, 308 IPA-Hexane, $.7 \mathrm{~mL} / \mathrm{min}, 254 \mathrm{~nm}$ 


\section{(S)-2-(2-Fluorophenyl)-6-phenyl-5,6-dihydro-4H-1,3-thiazin-4-one (3ad)}
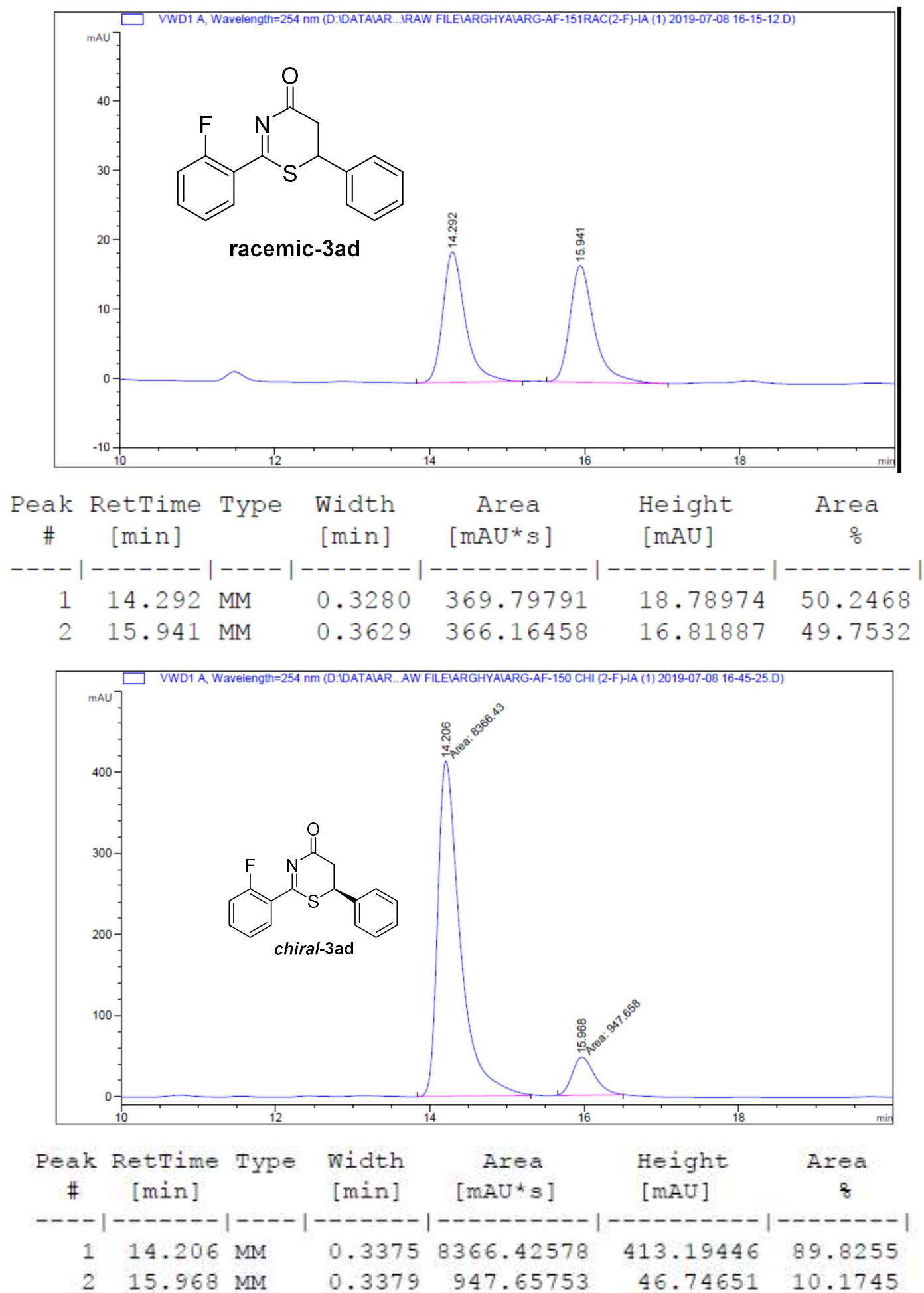

Sample Info: CHIRALPAR IA, 208 IPA-Hexane, $.7 \mathrm{~mL} / \mathrm{min}, 254 \mathrm{~nm}$ 


\section{(S)-2-(Naphthalen-1-yl)-6-phenyl-5,6-dihydro-4H-1,3-thiazin-4-one (3ae)}
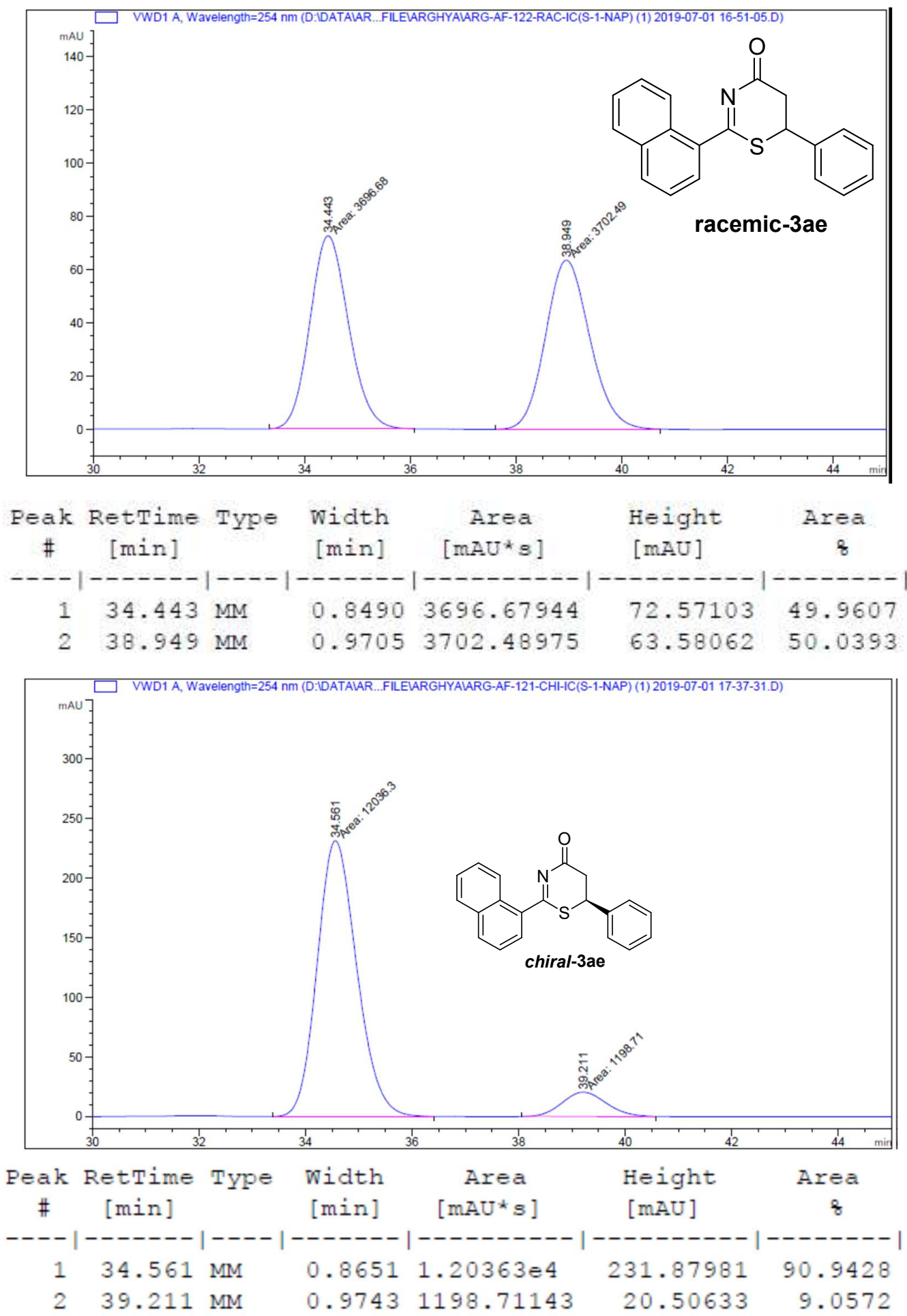

Sample Info: CHIRALPAK IC, 408 IPA-Hexane, $.7 \mathrm{~mL} / \mathrm{min}, 254 \mathrm{~nm}$ 


\section{(S)-6-Phenyl-2-(thiophen-2-yl)-5,6-dihydro-4H-1,3-thiazin-4-one (3af)}
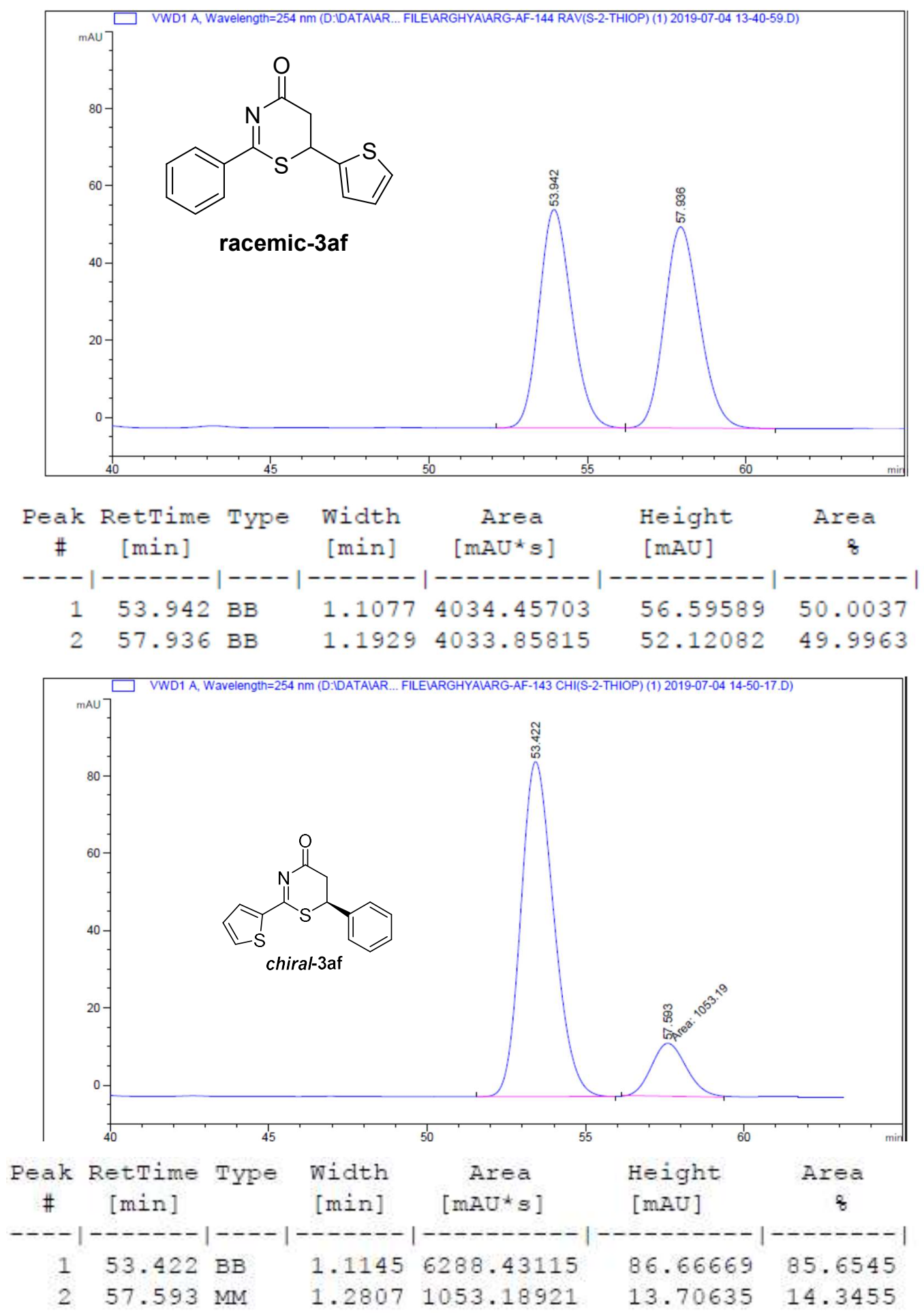

Sample Info: CHIRALPAK IC, 308 IPA-Hexane, $.7 \mathrm{~mL} / \mathrm{min}, 254 \mathrm{~nm}$ 